LBL--17IIS

DERA 005715

\title{
STRUCTURE OF PLANT BILE PIGMENTS
}

\author{
Robert William Schoenleber
}

(Ph.D. Thesis)

\author{
Lawrence Berke]ey Laboratory \\ University of Cal ifornia \\ Berkeley, California 94720
}

December 1983

\section{DISCLAIMER}

\begin{abstract}
This report was prepared as an account of work sponsored by an agency of the United States Eovernment. Neither the United States Government nor any agency thereof, nor any of their employees, makes any warranty, express or implied, or assumes any legal liability or responsibility for the accuracy, completeness, or usefulness of any information, apparatus, product, or process disclosed, or represents that its use would not infringe privately owned rights. Reference herein to any specific commercial product, process, or service by trade name, trademark, manufacturer, or otherwise does not necessarily constitute or imply its endorsement, recommendation, or favoring by the United States Government or any agency thereol. The views and opiniors of authors expressed herein do not necessarily state or reflect those of the United States Government or any agency thereof.
\end{abstract}

This work was supported in part by the Division of 8iological Energy Conversion and Conservation, U.S. Department of Energy under Contract No. DE-AC03-76SF00098, the National Institute of General Medical Sciences, DHHW, grant GM 28994, the National Science Foundation grant NSF PCM 82-08158, the National Science Foundation grant CHE 79-04832, and NIH Division of Research Resources grant RR01614. 
1

"Structure of Plant Bile Pigments"

by

Robert William Schoenleber

Abstract

Selective peptide cleavage has provided a general procedure for the study of the structure, including stereochem try, of plant bile pigments. The information derived from the synthesis and spectral analysis of a series of 2,3-dihydrodioxobilins allows the determination of the trans relative stereochemistry for ring $A$ of the $B_{p}$-phycocyanobilin from $C$-phycocyanin as well as for ring $A$ of phytocinrome. A complete structure proof of the five phycoarythrobilins attache: to the $\alpha$ and $\beta$ subunits of B-phycoerythrin is described. One of these etrapyrroles is dowdy-peptide linked to a single peptide chain through two thioethers at the $C-3^{\prime}$ and $C-13^{\prime}$ positions. The four remaining phycoerythrobilirs are singly-linked to the protein through thioethers at the $\mathrm{C}-3^{\prime}$ position and all possess the probable stereochemistry $C-2(R), C-3(R), C-3^{\prime}(R)$, and $C-16(R)$.

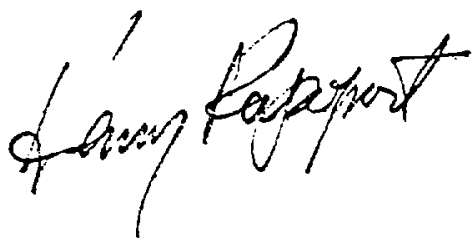




\section{Table of Contents}

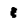

Page

Part One. The ReTative Stereochemistry of the A Ring

of Plant Bile Figments.................... 1

Part Two. Bilin Attednient Sites in the $\alpha$ and $\beta$ Subunits

of $y$-pryccerythrin: Structurat Studies on the

singly-Linked Phycoerythrobilins.............. 37

Part Three. Bilin Attachment Sites in the $\alpha$ ard $\&$ Subunits

of B-Phycoerythrin: Structural Studies on a

Doubly-Peptide Linked Phycoerythrobilin........... 74 


\section{Acknowledgment}

I thank Dr. Rapoport for his support and guidance, and Alex. Glazer for his enthusiasm, throughout the course of this work. Co-workers Dan Lunde 11, SeeLap Leung, and Youseung Kim deserve credit for much of this work.

I am especially grateful to Cheryl, Ann, and Jim. They are my source of strength. My good friend John helped me maintain a good attitude during this difficult time. And finally, I want to thank Teddy and Kazumi for their companionship during the final stages of this work. 
Dedication

To my parents.... 
Part One

The Relative Stereochemistry of the A Ring of Plant Bile Pigments

\section{Abstract}

The synthesis and characterization, including the stereochemistry, of a series of 3,4-dihydropyrromethenones and 2,3-dihydrodioxobilins are described. High resolution ${ }^{H} H$ NMR spectral analysis allows the determination of the A ring coupling constants for a series of cis and trans model compounds. From these data and correlations, the relative stereochemistry in the A ring of phycocyanin and similar bile pigment structures can be concluded. 
Complete structure elucidation of plant bile pigments (1) has been limited by the common practice of cleaving the linea i tetrapyrrole (3) from its covalently bound protein (Scheme I). ${ }^{1-6}$ This process has been a valuable tool for gross structural delerinination, as the resulting product can be easily studied by spectroscopy, ${ }^{7}$ chemical degradation, ${ }^{8}$ and total synthesis. ${ }^{9,10}$ The approach, however, suffers from several disadvantages. Firstly, all direct information on the chromophore-protein linkage, including two stereo centers, is lost. ${ }^{1}$ Secondly, side reactions leading to complex mixtures of unnatural chromophores have been observed. 11 Finally, the yield in tire cleavage step is frequently low. ${ }^{4,5}$ These limitations, when combinn with the fact that complex proteins are involved containing mull, . .e chromophores with possible structural variations, indicate a more controlled method for structural study is necessary.

In previous studies, we have developed methodology to conclusively ascertain the nature of the protein-tetrapyrrole covalent bond. ${ }^{12-14}$ By inild, selective degradation of the protein moiety without alteration of the chromophore or its attachment site, the three chiral centers about the A ring of the bile pigment are retained (Scheme I). Therefore, we are in a unique position to study the stereochemistry at these centers. For simplicity, we have chosen to concentrate on the phycocyanin series of bile pigments, as it contains only three chiral centers, all contigucus about the $A$ ring.

The proposed structure for the peptide-pigncst from the $\mathrm{N}$-terminal portion of the $\hat{b}$-suidunit of $\underline{C}$-phycocyanin from Synechococcus sp. 0301 is shown in structure 2. ${ }^{12}$ Assignment of stereocisemistry at $\mathrm{C}-2$ is based on the report that chromic acid oxidation of $\underline{C}$-phycocjenin results in the 
Scheme I. Bile Pigment Degradation Approaches.
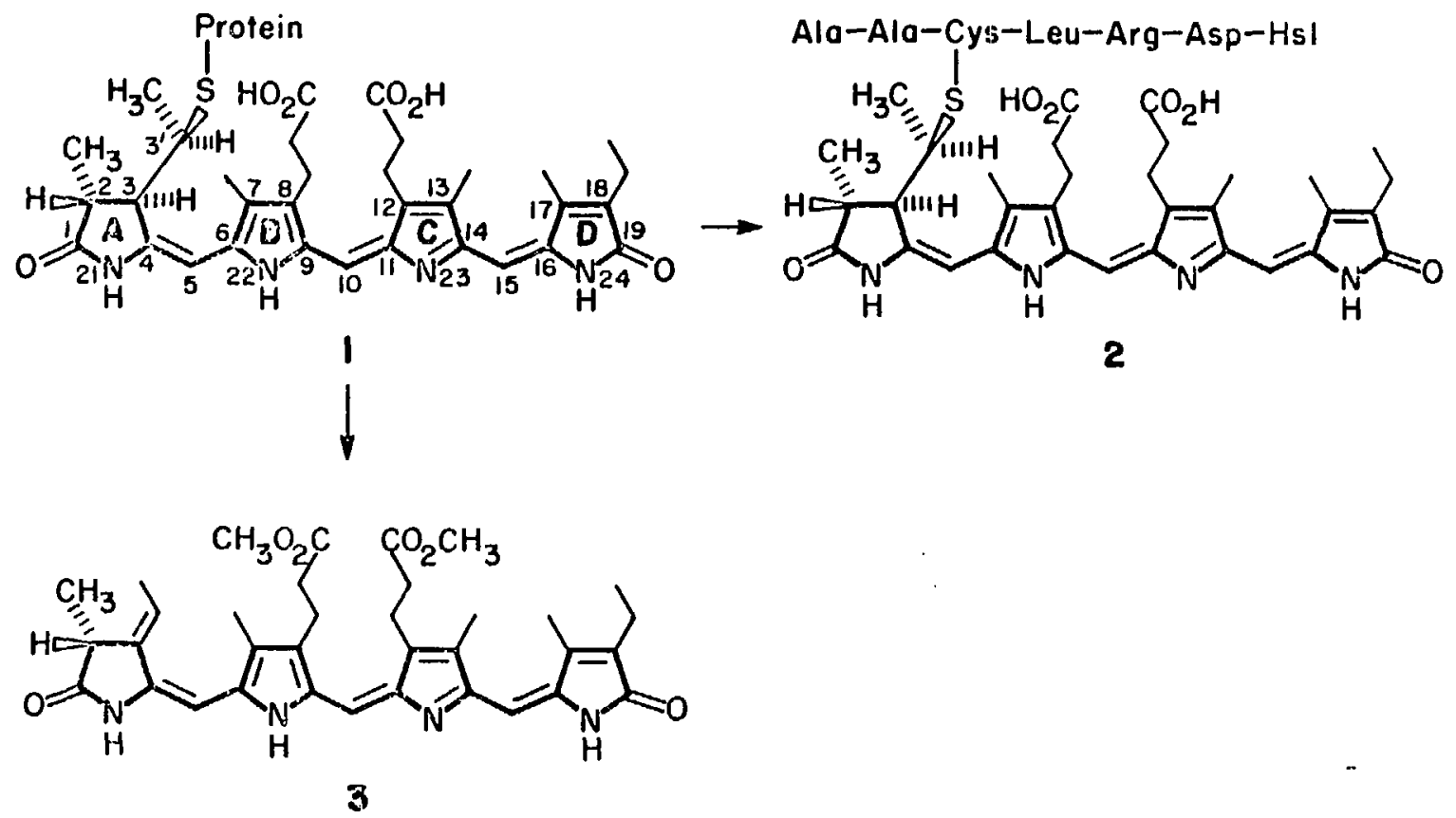
formation of $(E)-2-e t h y l$ idene-3(R)-methyl succinimide. ${ }^{15}$ Unfortunately, the actual analytical and spectroscopic data for the conpound obtained from the bile pigment degradation are unavailable in the literature. A750, C-phycocyanin contains three phycocyanobilins, each with possible structural differences. ${ }^{16}$ Although it seems reasonable that the three chromophores have the same stereochemistry, until this determination is made for each chromophoric unit, the question remains unanswered.

Assignment of the relative sterecchemistry between $C-3$ and $C-3^{\prime}$ a.]so is based on degradative evidence. Mechanistic interpretations indicate that an antiperiplanar elimination of the sulfone resulting from thioether oxidation gives the observed $(E)$-ethylidene succinimide. ${ }^{17}$ Although the findirigs have been corraborated by thiol el imination to the same product on numerous occasions, no detailed mechanistic studies on this reaction have been done. ${ }^{15}$ Despite the evidence presented, the multiple chromophore problem casts doubts on the utility of these data.

The remaining stereochemical relationship to be addressed, then, is the relative stereochemistry between the $\mathrm{C}-2$ and $\mathrm{C}-3$ centers. Having two adjacent protons in a rigid five membered ring suggests as a solution the homonuclear coupling constant determination of the dihedral angle relationship. Conclusions would require the proper model compounds, as the system is too complex to approach theoretically. ${ }^{18}$ Although some model systems are available, the information cannot be directly related to the natural product. ${ }^{17,19}$ Possible solvent effects and complicating side chain functionality have prevented a definitive interpretation.

We present a systematic study of model 2,3-dihydrodioxobilins and correlate their NMR properties to shose of natural bile pigments. The information gained, if used with properly collected degradative data, 
should allow the complete absolute stereochemical assigrment of any phycocyanobilipeptide.

Our initial synchetic goal then, was to gain entry into symetrically substituted octaalkyl dihydrobiliverdins (Scheme II). The octaethyl series was chosen to avoid any solubility problenis which might restrict the analysis. We began with the 1,3-dipolar addition of the tosylmethylisocyanide anion to an $\alpha, \beta$-unsaturated ketone that allows for rapid and facile entry into 3,4-dialkylpyrroles, after reduction of the carbonyl group. ${ }^{20,21}$ The 3,4-diethylpyrrole (4) thus obtained served as the starting material for all four pyrrolic rings. This pyrrole could be formylated under Vilsmeier conditions to give an appropriately functionalized unit 6 for the $B$ and $C$ rings. ${ }^{22}$ The diethylpyrrole could also be oxidized in a stepwise manner, first to give 3,4-diethyl-3-pyrrolin-2-cne (5) and then further to give 3,4-dimethylmaleimide. 22,23 The pyrrolinone was the $A$ and $D$ ring precursor, while the maleimide was a necessary compound for the stucture proof.

Sase catalyzed condensation of pyrrolinone 5 and formylpyrrole 6 gave $3,3^{\prime}, 4,4^{\prime}$-tetraethyl-5(1H)-pyrromethenone $(7)$, again a commen intermediate. ${ }^{22}$ Formylation, this time with trimethyl orthoformate gave the key pyrromethenone 8 into which the 3,4-dihydro feature could be introduced. ${ }^{10}$ This catalytic reduction of the 5'-formy 7 pyrromethenorie 8 gave a mixture of products reduced at the 3,4- and the methine double bonds, as well as the tetrahydro material. 24,25 The desired 3,4-dihycro compound was the major product and could be easily separated by chrometography. Cis-3,4-dihydro-5'-formylpyrrometherone (9) obtained from the catalytic reduction was epinierized completely to the trans isomer by refluxing in methanolic sodium hydroxide. ${ }^{24}$ Operationally, it is ensier 
Scheme 11. Synthesis of cis- and trans-2,3-Dihydrooctaethy1biliverdins 11 and 12 .

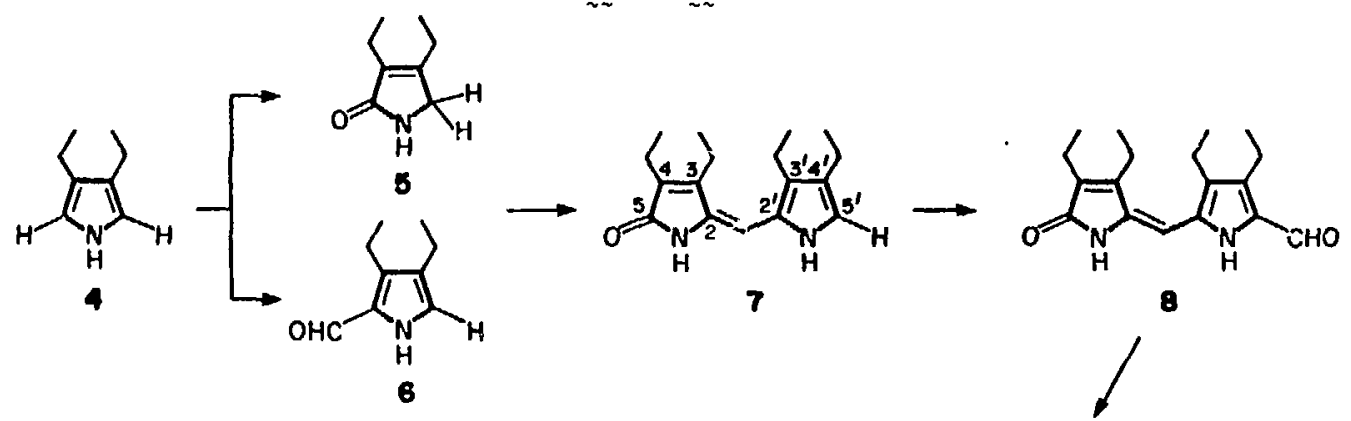

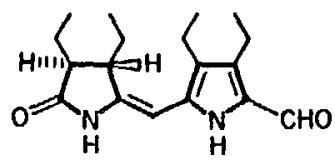

10

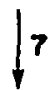

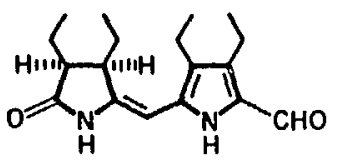

9

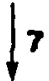

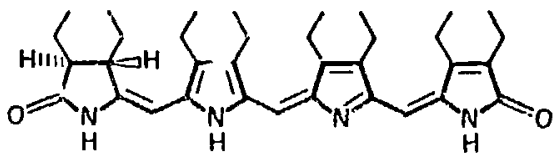

12

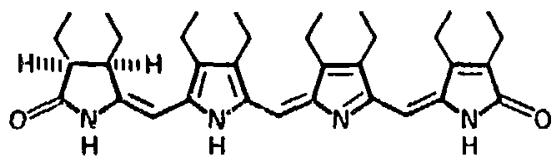

If 
to carry out the epimerization on the crude reduction mixture and then do a single purification to obtain the trans compound.

The final synthetic transformation in this series was to couple the aldellydic pyrromethenone $\underset{\sim}{g}$ and $\underset{\sim \sim}{10}$ with the $5^{\prime}-H$ pyrroinethenone 7 to give the dioxotetrapyrroles 11 and 12 without epimerization at either $\mathrm{C}-2$ or C-3. ${ }^{26}$ A fi.ial HPLC of purification of the resulting product showed that no epimerization had occurred, especially if one uses only $33 \mathrm{mol \%}$ of $\mathrm{POBr}_{3}$ and 100 mol\% of 2,5-di-t-butyl-4-methylpyridine for the condensation reaction.

Al though the octaalkyl series gave us a valid 2,3-dihydro model system, a more appropriately functionalized linear tetrapyrrole was necessary for direct correlation with the natural pigments. Due to the inherent dissymmetry of the bile pigments, a more elaborate synthetic scheme to the ring precursors was required. However, the synthesis is still quite convergent, and the key synthetic transformations to obtain the 2,3-dihydro feature and to couple to the uitimate dioxotetrapyrroles were retained.

The essary pyrrolinones $13 \mathrm{a}$ and $13 \mathrm{~b}$ were synthesizeci through their corrcsponding cyanohydrins (Scheme III). ${ }^{27}$ We found that the intemediacy of the bisulfite adduct avoided the need for large amounts of anhydrous HCN. ${ }^{28}$ Attempts at reducing the cyanolydrin with platinum failed, however, the now standard Raney nickel reduction led smoothly to the desired compounds. 27

The formyl pyrrole 14 came from an initial Knorr condensation followed by extensive functional group transformations to obtain the necesiary substituent patter.. 29,30 Base catalyzed condensation to pyrronethenones 15 was done in methanclic KCH as in the ethyl series 
Scheme III. Synthesis of Ethylmethylpyrrolinones 13a and 13b.<smiles>[R]C(=O)C([R2])C(=O)OCC</smiles>

a, $R_{1}=\mathrm{C}_{2} \mathrm{H}_{5}, \mathrm{R}_{2}=\mathrm{CH}_{3}$ b, $\mathrm{R}_{1}=\mathrm{CH}_{3}, \mathrm{R}_{2}=\mathrm{C}_{2} \mathrm{H}_{5}$

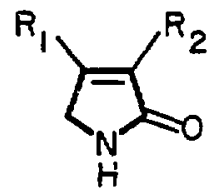

$13 a, R_{1}=C_{2} H_{5}, R_{2}=C H_{3}$ $13 \mathrm{~b}, \mathrm{R}_{1}=\mathrm{CH}_{3}, \mathrm{R}_{2}=\mathrm{C}_{2} \mathrm{H}_{5}$

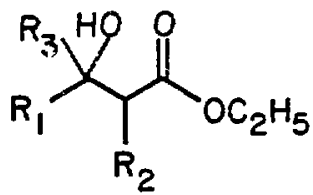

$$
\mathrm{R}_{3}=\mathrm{SO}_{3} \mathrm{H}, \mathrm{CN}
$$
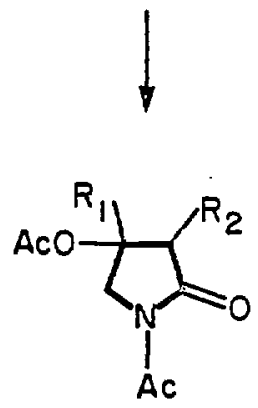
(Scheme IV). ${ }^{30}$ This required a subsequent re-esterification with diazomethane. Attempts to carry out the condensation with sodium. methoxide resulted in significantly lower yields.

The $5^{1}$-t-tutoxycarbonyl group served as a stabilizing group during the pyrrole synthesis. Although catalytic reduction to the dihydro series can be done on the ester, ${ }^{24}$ we wanted to minimize the number of transformations after the reduction in order to avoid exposure to epimerization. Therefore, an acid catalyzed decarboxylative-formylation was used a.d gave the 5'-formylpyrromethenone 16 directly. On the other hand, treatment of the t-butyl ester with neat triflurooacetic acid for five minutes allows one to isolate the $5^{\prime}$-acid in good yield, while a longer reaction time (two hours) effects decarboxylation to give the $5^{\prime}-\mathrm{H}$-pyrromethenone 17. This latter compound is the precursor of the C,D-ring moiety.

The remainder of the synthesis was directly analogous to the ethyl series, and led to the synthesis of the four 2,3-dihydrodioxobilins $20 \mathrm{a}$, 20b, $\underset{\sim}{21 \mathrm{a}}$, and ${\underset{\sim}{2} \mathrm{~b}}_{\sim}$ as shown in Scheme IV. The side chain methyl ester introduced some problems, as some hydrolysis was seen in both the cpimerization and coupling steps. However, in both cases the acid could easily be separated from the ester and re-esterified with stoichiometric diazomethane. The final products were purified by HPLC, and again the coupling was effected without epimerization.

It has always been assumed that catalytic hydrogenation gives cis hydrogen addition to the pyrromethenones. Hovever, the empirical nature of the experimental results makes such a simplistic assumption tenuous. Since the methine double bond is trisubstituted while the 3,4-double bond is tetrasubstituted, it is surprising that the 3,4-dihydro compound 
Scheme IV. Synthes is of 2,3-Dihydrodioxobilins $\underset{\sim \sim \sim}{20 \mathrm{a}}, \underset{\sim \sim \sim}{20 \mathrm{~b}}, \underset{\sim}{21 \mathrm{a}}$, and $\underset{\sim \sim}{21 \mathrm{~b}}$.<smiles>[R]C1=C([R])C(=O)NC1</smiles>

13

14

15
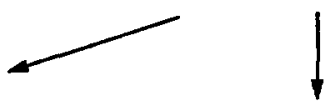

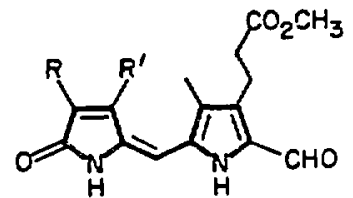

16

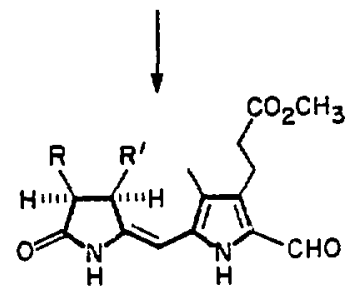

18

$17 b$

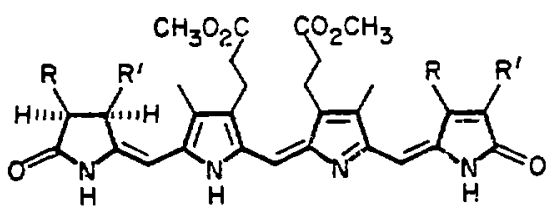

20

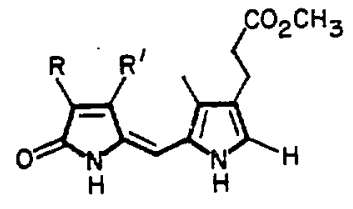

17

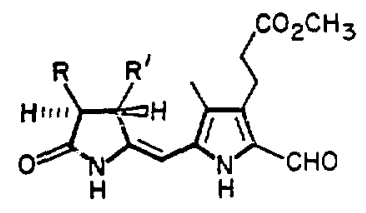

19

170

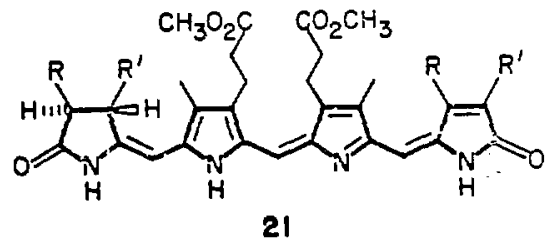

$$
\begin{aligned}
& a, R=C_{3}, R^{\prime}=C_{2} H_{5} \\
& \text { b. } R=C_{2} H_{5}, R^{\prime}=C_{3} H_{3}
\end{aligned}
$$


is the major product. Furtherimore, if the reduction is done under alkaline conditions or with other catalysts, only the pyrroliethane is seen. 24 Due to uncertain mechanistic paths in such a complex system, we concluded that a more rigorous stereochemical proof was required in our dioxobilin series.

Diethylmaleimide and ethylmethylmaleinide were independently synthesized by oxidation of the corresponding pyrroles. ${ }^{22,23}$ Catalytic hydrogenation of these compounds over $\mathrm{PtO}_{2}$ gives the cis-succinimide in nearly quantitative yield. ${ }^{31}$ The trans-succinimides were then obtainable from a strong base-catalyzed epimerization.

Each of the dioxobilins was then subjected to the now standard chromic acid oxidation. ${ }^{32}$ In the octaethyl series, diethylmaleimide was generated from the $B, C$, and $D$ rings. From the trans-tetrapyrrole, only the trans-succinimide resulting from $A$ ring oxidation was seen by $G C$ analysis; no cis-succinimide was present. The cis-tetrapyrrole likewise gave diethylmaleimide and only cis-diethylsuccinimide. Although no rigorous detection limits were established, less than ten percent of the isomeric succinimide would easily have been seen in each case.

The bile pigment degradation products are more easily observed by TLC analysis, using the sensitive chlorine/o-anisidine reagent for visualization. ${ }^{33,34}$ Chromic acid oxidation of each of the four compounds $20 \mathrm{a}, 20 \mathrm{~b}, 21 \mathrm{a}$ and $\underset{\sim}{21 \mathrm{~b}}$ gave poor yields, as usual, but no interfering side products. Each dioxotetrapyrrole gave positive tests for ethylmethylmaleimide and hematinic acid methyl ester, resulting from the $B$, $C$, and $D$ rings. From both trans-tetrapyrroles $\underset{\sim}{21 a}$ and $\underset{\sim}{216}$ only transethylmethylsuccinimide was obtained. The cis-tetrapyrroles $\underset{\sim}{20 \mathrm{a}}$ and $\underset{\sim}{20 \mathrm{~b}}$ gave cis-ethylnethylsuccinimide with a barely detectable trace of the 
trans succinimide. Considering the sensitivity of the visualization process, the trace amounts are extremely smal1. Since the dioxotetrapyrroles $\underset{\sim}{20 \mathrm{a}}$ and $\underset{\sim \sim}{20 \mathrm{~b}}$, having been purified by preparative HPLC prior to degradation, were free from contaminating trans-isomer, this indicates that a small amount of epimerization did occur in the axidation step. ${ }^{35}$ The results, however, leave no doubt that the correct cis and trans assignments have been made.

The $I_{H}$ NMP. assignments for the cis and trans-3,4-dihydro-5'-formy 1 pyrromethenones $18 \mathrm{a}, \underset{\sim \sim \sim}{18 b}, \underset{\sim \sim}{19 a}$, and $\underset{\sim \sim \sim}{19 b}$ in $\mathrm{CDCl}_{3}$ are shown in Table I. Decoupling experiments allowed us to assign all proton resonances and to determine the $3-\mathrm{H} / 4-\mathrm{H}$ coupling constants (Table IV) despite a few overlapping resonances. This analysis greatly facilitated the interpretation of the more complicated tetrapyrrole spectra.

The ${ }^{\prime} H$ NMR assignments for the dioxotetrapyrroles 20 and 21 in $\mathrm{COCl}_{3}$, along with shifts reported in a previous study, $25^{\sim}$ are found in Table II. Compound 21b is included in this study for completeness, as we belleve that conclusive evidence for all bile pigment attachments through the $A$ ring has yet to be presented. Our values agree well $(0.05 \mathrm{ppm})$ with those reported ${ }^{25}$ with the exception of the $\mathrm{C}-2-\mathrm{H}$, $\mathrm{C}-2-\mathrm{CH}_{3}$, and $\mathrm{C}-3-\mathrm{H}$. These resonances show the most variation when comparing the pyrromethenones and the dioxotetrapyrroles as we11. The decoupling experiments, though, leave no doubt about the assignments made. The chemical shift variation may reflect differing protonation states due to trace amounts of acid in the solverit or concentration effects, as these noiecules are known to exhibit complex solution conformational behavior.

In our study of the $B_{1}$-phycocyanobiliheptapeptide, ${ }^{12}$ we found 
Table I. 'H NMR Assignments for the 3,4-Dihydro-5'-formyl cis (18) and trans (19) Pyrromethenones in $\mathrm{COCl}_{3}$.

\begin{tabular}{|c|c|c|c|c|}
\hline assignment & 18a (is) & $\underset{\sim}{18 b}$ (cis) & $19 \mathrm{a}(\operatorname{trans})$ & $19 \mathrm{~b}$ (trans) \\
\hline $5^{\prime}-\mathrm{CHO}$ & 9.54 & 9.54 & 9.54 & 9.53 \\
\hline meso-H & 5.37 & 5.35 & 5.31 & 5.31 \\
\hline $4^{\prime}-\mathrm{CH}_{2} \mathrm{CH}_{2} \mathrm{CO}_{2} \mathrm{CH}_{3}$ & 3.68 & 3.68 & 3.68 & 3.67 \\
\hline $4^{\prime}-\mathrm{CH}_{2} \mathrm{CH}_{2} \mathrm{CO}_{2} \mathrm{CH}_{3}$ & 3.06 & 3.10 & 3.06 & 3.06 \\
\hline $3-\mathrm{H}$ & 2.95 & 3.26 & 2.65 & 2.85 \\
\hline $4^{\prime}-\mathrm{CH}_{2} \mathrm{CH}_{2} \mathrm{CO}_{2} \mathrm{CH}_{3}$ & 2.58 & 2.57 & 2.58 & 2.58 \\
\hline $4-H$ & 2.79 & 3.05 & 2.42 & 2.17 \\
\hline $3^{\prime}-\mathrm{CH}_{3}$ & 2.03 & 2.02 & 2.02 & 2.03 \\
\hline 3 or $4-\mathrm{CH}_{2} \mathrm{CH}_{3}$ & $1.6-1.8$ & $1.5-2.0$ & $1.5-2.0$ & $1.5-2.0$ \\
\hline 3 or $4-\mathrm{CH}_{3}$ & 1.21 & 1.23 & 1.32 & 1.36 \\
\hline 3 or $4-\mathrm{CH}_{2} \mathrm{CH}_{3}$ & 1.01 & 1.05 & 1.02 & 1.03 \\
\hline
\end{tabular}


Table 11. 1H MMR Assignments for the 2,3-Dihydrodioxobilins 20 (cis) and 21 (trans) in $\mathrm{COCl}_{3}$.

\begin{tabular}{|c|c|c|c|c|}
\hline assignment & 20a (cis) & $20 \mathrm{~b}$ (cis) & 2la (trans) & $21 \mathrm{~b}$ (trans) \\
\hline $10-H$ & $6.65(6.64)^{\mathrm{a}}$ & $6.64(6.65)$ & 6.64 & $6.65(6.66)$ \\
\hline $15-h$ & $5.98(5.98)$ & $6.00(6.01)$ & 5.98 & $6.01(6.01)$ \\
\hline $5-\mathrm{H}$ & $5.49(5.47)$ & $5.47(5.47)$ & 5.46 & $5.47(5.48)$ \\
\hline \multirow{3}{*}{$8,12-\mathrm{CH}_{2} \mathrm{CH}_{2} \mathrm{CO}_{2} \mathrm{CH}_{3}$} & $3.68(3.68)$ & $3.68(3.68)$ & 3.68 & $3.68(3.68)$ \\
\hline & $3.66(3.67)$ & $3.67(3.67)$ & 3.67 & $3.67(3.67)$ \\
\hline & $2.94(2.94)$ & $2.94(2.93)$ & 2.94 & $2.95(2.92)$ \\
\hline $8,12-\mathrm{CH}_{-2} \mathrm{CH}_{2} \mathrm{CO}_{2} \mathrm{CH}_{3}$ & $2.90(2.90)$ & $2.90(2.93)$ & 2.90 & $2.90(2.92)$ \\
\hline \multirow[t]{2}{*}{$3-4$} & $3.14(2.75)$ & $3.38(2.93)$ & 2.79 & $2.94(2.14)$ \\
\hline & $2.55(2.55)$ & $2.55(2.54)$ & 2.55 & $2.56(2.54)$ \\
\hline \multirow{2}{*}{$\begin{array}{l}\mathrm{B}, 12-\mathrm{CH}_{2} \mathrm{CH}_{2} \mathrm{CO}_{2} \mathrm{CH}_{3} \\
2-\mathrm{H}\end{array}$} & $2.54(2.54)$ & $2.54(254)$ & 2.54 & $2.54(2.54)$ \\
\hline & $2.85(2.32)$ & $2.6^{b}(2.1)$ & $2.35^{\mathrm{b}}$ & $2 . .5^{b}(2.92)$ \\
\hline \multirow{2}{*}{17 or $18-\mathrm{CH}_{2} \mathrm{CH}_{3}$} & $2.32(2.32)$ & $2.55^{b}(2.54)$ & 2.32 & $2.55^{\mathrm{b}}(2.54)$ \\
\hline & $(2.12(2.12)$ & $2.12(2.12)$ & 2.12 & $2.12(2.12)$ \\
\hline \multirow{2}{*}{$7,13,17$ or $18-\mathrm{CH}_{3}$} & $2.11(2.12)$ & $2.02(2.02)$ & 2.11 & $2.02(2.02)$ \\
\hline & $2.02(\overline{c .02)}$ & $1.85(1.84)$ & 2.01 & $1.85(1.85)$ \\
\hline 2 or $3-\mathrm{CH}_{-2} \mathrm{CH}_{3}$ & $1.5-2.0(1.7-2.0)$ & $1.4-2.0(1.6-1.8)$ & $1.6-2.0$ & $1.5-1.9(1.6-1.8)$ \\
\hline 2 or $3-\mathrm{CH}_{3}$ & $1.12(1.24)$ & $1.27(1.42)$ & 1.24 & $1.43(1.43)$ \\
\hline 17 or $18-\mathrm{CH}_{2} \mathrm{CH}_{3}$ & $1.10(1.10)$ & $1.22(1.22)$ & 1.10 & $1.22(1.22)$ \\
\hline 2 or $3-\mathrm{CH}_{2} \mathrm{CH}_{-3}$ & $1.01(1.06)$ & $1.02(0.99)$ & 1.06 & $0.99(0.99)$ \\
\hline
\end{tabular}

${ }^{a}$ Chemical shifts in parentheses are from reference 25 .

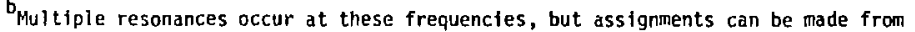
decrupling and integration studies. 
pyridine to be an excellent solvent for avoiding aggregation and conformational effects. The basicity of the pyridine, present in ląrge excess, preciudes differential protonation of the substrate, and the aromatic nature of this solvent encourages intercalation between substrate molecules, preventing aggregation. Thus, the conformations adopted by the dioxotetrapyrroles appear to be uniform.

To complete our present study, therefore, the $360 \mathrm{MHz}{ }^{1} \mathrm{H}$ MMR spectra of tine dioxobilins were recorded in pyridine-d $d_{5}$ (Table III). Not only does this serve to avoid the problems mentioned above, but it also allcws us to make a direct comparison with previous natural products studied in pyridine. There is much less variation in resonances for the model compounds and several patterns appear. The $\mathrm{C}-15-\mathrm{H}$ in the 2-ethyl3-methyl series is shifted downfield approxinately $0.1 \mathrm{ppm}$ from that in the 3-ethyl-2-methyl series. The A ring methyl group for the former series is also shifted downfield $0.1 \mathrm{ppm}$. Finally, although the $\mathrm{C}-2-\mathrm{H}$ and $\mathrm{C}-3-\mathrm{H}$ still show the most variation in chemical shift, the 2-ethyl-3-methyl cis and trans compounds show a significant $(0.2-0.3 \mathrm{ppm})$ upfield shift of the [-2-H as compared to the corresponding 3-ethy]-2-methyl compounds.

That our modeis truly reflect the actual case is shown by the excellent agreement between the shifts for $21 \mathrm{a}$, the most likely model, and the $B_{1}$-phycocyanobiliheptapeptide 2. Where minimal perturbation of structure is seen, the agreement is within $0.05 \mathrm{ppm}$. Of particular importance is the correlation of the $\mathrm{C}-2-\mathrm{CH}_{3}$ and even the $\mathrm{C}-2-i$. The substituents on the $B, C$, and $D$ rings likewise agree well, al though the presence of the acid rather than the ester causes slightly larger shift differences in the propionic acid resonances. The riethine protons aiso show a substantial difference in shift as has been seen before when 


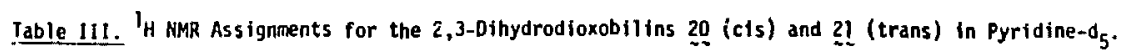

\begin{tabular}{|c|c|c|c|c|}
\hline assignment & $20 \mathrm{a}(c i s)$ & $200(c i s)$ & 21a (trans) & 21b (trans) \\
\hline $10-H$ & 7.09 & 7.07 & $7.08(7.29)^{\mathrm{d}}$ & 7.09 \\
\hline $15-H$ & 6,07 & 6.15 & $6.07(6.08)$ & 6.17 \\
\hline \multirow[t]{2}{*}{$5-H$} & 5.47 & 5.47 & $5.44(5.87)$ & 5.47 \\
\hline & 3.64 & 3.64 & 3.64 (b) & 3.64 \\
\hline \multirow{2}{*}{$8,12-\mathrm{CH}_{2} \mathrm{CH}_{2} \mathrm{CO}_{2} \mathrm{CH}_{-3}$} & 3.62 & 3.62 & 3.62 (b) & 3.62 \\
\hline & 3.09 & 3.08 & $3.09(3.17)^{b}$ & 3.09 \\
\hline $8,12-\mathrm{CH}_{-2} \mathrm{CH}_{2} \mathrm{CO}_{2} \mathrm{CH}_{3}$ & 3.00 & 3.00 & $3.00(3.00)^{b}$ & 3.00 \\
\hline \multirow[t]{2}{*}{$3-H$} & c & 3.28 & $2.7^{\mathrm{d}}(3.15)^{\mathrm{b}}$ & 2.93 \\
\hline & 2.72 & 2.72 & $2.71(2.85)^{b}$ & 2.72 \\
\hline \multirow{2}{*}{$8,12-\mathrm{CH}_{2} \mathrm{CH}_{2} \mathrm{CO}_{2} \mathrm{CH}_{3}$} & 2.69 & 2.68 & $2.69(2.83)^{b}$ & 2.69 \\
\hline & $3.03^{d}$ & 2.71 & $2.62(2.70)$ & 2.40 \\
\hline \multirow{2}{*}{17 or $18-\mathrm{CH}_{2} \mathrm{CH}_{3}$} & 2.52 & 2.55 & $2.51(2.48)$ & 2.55 \\
\hline & 2.11 & 2.10 & $2.11(2.12)$ & 2.11 \\
\hline \multirow{2}{*}{$7,13,17$ or $18-\mathrm{CH}_{3}$} & 2.09 & 2.05 & $2.09(2.07)$ & 2.05 \\
\hline & 1.95 & 1.94 & $1.96(2.02)$ & 1.96 \\
\hline 2 or $3-\mathrm{CH}_{2} \mathrm{CH}_{3}$ & $1.6-1.7$ & $1.4-1.7$ & $1.6-2.0(e)$ & $1.6-2.0$ \\
\hline 2 or $3-\mathrm{CH}_{3}$ & 1.24 & 1.33 & $1.36(1.39)$ & 1.47 \\
\hline 17 or $18 \cdot \mathrm{CH}_{2} \mathrm{CH}_{3}$ & 1.30 & 1.24 & $1.28(1.23)$ & 1.24 \\
\hline 2 or $3-\mathrm{CH}_{2} \mathrm{CH}_{3}$ & 0.97 & 1.08 & $1.03(1.48)^{\mathrm{e}}$ & 1.03 \\
\hline \multicolumn{5}{|c|}{ 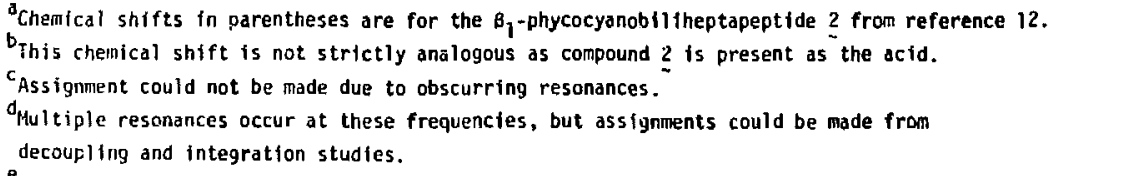 } \\
\hline
\end{tabular}


comparing a free dioxotetrapyrrole to one which is peptide bound. ${ }^{12}$ The nature of this difference is still unknown and, to be deciphered, iwill require synthesis of a more appropriate model incorporating the amino acid thioether moiety. The presence of the thioether likewise destroys any possible comparison at the $\mathrm{C}-3-\mathrm{H}$ or any of the $[-3$-ethyl protons.

The most important stereochemical information comes from the pattern of A ring coupling constants seen in Table IV. The $J_{3,4}$ for the cisdihydropyrromethenones 18 centers around $8.1 \mathrm{~Hz}$ in $\mathrm{CDCl}_{3}$ while the trans compounds $\underset{\sim}{19}$ show $\mathrm{J}_{3,4} \simeq 5.0 \mathrm{~Hz}$. The ethyl compounds show coupling constants quite a bit lower $(6.8$ and $2.9 \mathrm{~Hz}$, respectively) due to the steric interaction between the $\mathrm{C}-3$ and $\mathrm{C}-3$ ' ethyl groups which must distort the conformation. The dioxotetrapyrroles show slightly higher $J$ values, as now the tendency to form helical conformations removes some of the $\mathrm{C}-3 / \mathrm{C}-7$ steric interaction. In pyridine, the coupling constants show the same behavior. For the cis-dioxotetrapyrroles 20 one sees $\mathrm{J}_{2,3} \simeq$ $8.3 \mathrm{~Hz}$ while the trans compounds $\underset{\sim}{21}$ exhibit $J_{2,3} \simeq 5.8 \mathrm{~Hz}$. The coupling constants are not nearly as sensitive to solvent effects as are the chemical shifts. Again, as witnessed by the ethyl series, steric bulk about the $\mathrm{C}-3$ and $\mathrm{C}-7$ centers causes a pronounced $(1.0-1.5 \mathrm{~Hz})$ lowering of the coupling constant.

The pattern is clear. Based on the models with the appropriate substitution, $\underset{\sim}{20 \mathrm{a}}$ and $\underset{\sim}{21 \mathrm{a}}$, one expects the $2-\mathrm{H} / 3-\mathrm{H}$ coupling constant in pyridine to be $8.2 \mathrm{~Hz}$ in the cis configuration and $5.7 \mathrm{~Hz}$ in the trans configuration. Returning to the $B_{1}$-phycocyanobiliheptapeptide, we observe a coupling constant of $5.0 \mathrm{~Hz}$ for these protons. ${ }^{12}$ As the steric effect of the thioether undoubtedly causes a slight lowering of the coupling constant, we can now conclusively state that this bile 
Table IV. ${ }^{1}$ H Coupling Constants ${ }^{\mathrm{a}}$ observed for Ring A.

\begin{tabular}{|c|c|c|c|}
\hline compound & stereochemistry & $\mathrm{J}\left(\mathrm{Hz}, \mathrm{CDCl}_{3}\right)$ & $J\left(H z\right.$, pyridine $\left.-d_{5}\right)$ \\
\hline $18 \mathrm{a}$ & cis & 8.2 & - \\
\hline $18 \mathrm{~b}$ & cis & 8.0 & - \\
\hline$\underset{\sim}{\underline{g}}$ & cis & 6.8 & - \\
\hline$\underset{\sim \sim \sim}{20 \mathrm{a}}$ & cis & 8.5 & 8.2 \\
\hline $20 \mathrm{~b}$ & cis & 8.2 & 8.5 \\
\hline 11 & cis & 6.5 & 7.6 \\
\hline$\underset{1 \tilde{1}}{\tilde{1}} \mathrm{a}$ & trans & 4.9 & - \\
\hline$\underset{\sim \sim \sim}{19 b}$ & trans & 5.1 & - \\
\hline 10 & trans & 2.9 & - \\
\hline $21 \mathrm{a}$ & trans & 5.4 & 5.7 \\
\hline $21 \mathrm{~b}$ & trans & 6.0 & 5.8 \\
\hline 12 & trans & 3.7 & 4.1 \\
\hline
\end{tabular}


pigmenit las a trans relationship between the $\mathrm{C}-2-\mathrm{H}$ and the $\mathrm{C}-3-\mathrm{H}$. Given the previously mentioned caveats in regard to tine assignmient of the stereochemistry at $\mathrm{C}-2$ and $\mathrm{C}-3^{\prime}$, our results also can serve to assign the absolute stereochemistry at the $C-2, C-3$, and $C-3^{\prime}$ centers as $R, R, R$. These data are applicable to the phytochrome structure as we11, as the pigment structure differs only in the substitution of a vinyl for an ethyi group at $\mathrm{C}-18$. The chemical shift correspondence substantiates this claim. ${ }^{13}$ Again, a coupling constant of $5.0 \mathrm{~Hz}$ was seen for the 2-H/3-H interaction. By direct analogy, a trans-dihydro A ring must be present. This completes the relative stereochenical assignment of the phytochrome tetrapyrrole. The absulute stereochemistry has yet to be determined; therefore, the possibilities for the $\mathrm{C}-2, \mathrm{C}-3$, and $\mathrm{C}-3^{\prime}$ centers remain $R, R, R$ or $S, S, S$.

Although drawing the analogy to the phycoerythrin as well as other bile pigment series would seem straightforward, it is clear that the stereochemistry at $\mathrm{C}-16$ has a profound effact on the spectroscopic properties of this chromophore. ${ }^{36}$ In our studies in this series, we found a $2-\mathrm{H} / 3-\mathrm{H}$ coupling constant of $3.5 \mathrm{~Hz}$ in $\mathrm{D}_{2} \mathrm{O}^{14}$ This low value is the first good evidence for the trans configuration in that series. However, because of possible solvent differences and until the phycoerythrin model series is synthesized, no stronger argument can be made. 


\section{Experimental Section}

General Methods. Tetrahydrofuran (THF) was distilled from sodium/ benzophenone, metharol was distilled from magnesium, methylene chloride was distilled from phosphorus pentoxide, $\underline{t}$-butanol and ethyl acetate were distilled from calcium hydride, pyridine was distilled from $\mathrm{p}-$ toluenesulfonyl chloride then from calcium hydride, and trimethyl or thoformate was freshly distilled before use. Potassium t-butoxide was freshly sublimed before use. Sodium methoxide was prepared imnediately before use, and all other reagents were made as referenced or used directly from commercial suppliers after verification.

Melting points were measured on a Buchi capillary melting point apparatus and are uncorrected. IR spectra were recorded on a PerkinElmer 137 spectrophotometer with polystyrene film for calibration (1601.4 $\mathrm{cm}^{-1}$ absorption). UV/vis spectra were recorded in methanol with a Perkin-Elmer 522A spectrophotometer. Unless otherwise noted, the ${ }_{H}$ NMR spectra were determined on the UCB-200 spectrometer (a home-made FT instrument operating at $201.95 \mathrm{Miz}$ ). The $I_{H}$ NMR spectra in pyridine-d $d_{5}$ were recorded on the University of California, Davis, $360 \mathrm{MHz}$ NMR spectrometer. All chemical shifts are expressed in parts per million ( $\delta$ ) downfield from an internal $\mathrm{Me}_{4} \mathrm{Si}$ standari. The tabulated chemical shifts were recorded on approximately $15 \mathrm{mM}$ samples. Multiplicities are not included for the sake of clarity, but are easily inferred froin the data given. High-resolution (exact mass) mass spectra were obtained on a Kratos MS-50. Elemental analyses were performed by the Analytical Laboratory, College of Chemistry, University of Califoinia, Berkeley.

Gas chromatography (GC) was done with a Hewlett-Packard 402 gas 
chromatograph with a he flow rate of $80-100 \mathrm{~mL} / \mathrm{min}$ on a $1.8 \mathrm{~m} 5 \%$ Dexsi1 300 on $90 / 100$ Anakroill Q columin ( $5 \mathrm{~mm}$ i.d.) operating at $160^{\circ} \mathrm{C}$. tigh pressure liquid chromatcgraphy (HPLC) was dnne on an Altex anaiytical system consisting of two Model 110A punps, a 155-10 UV/vis detector, and a Model 420 microprocessor controller/programmer. An Altex 10,250 mm, $10 \mu \mathrm{m}$ LiChrosorb Sifl silica gel column was used with detection at $340 \mathrm{~nm}$ with the following =olvent compositions $(v / v)$ : (A) $60 \%$; joctane/40\% ether; (B) $0.75 \%$ methansl/chloroforr; (C) $1.25 \%$ methanol/ch,iuroform; (D)

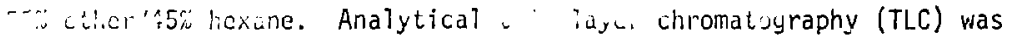
done on aluminum-backed silica plates activated at $100^{\circ} \mathrm{C}$ for 2 hours. A three-fold multipia development was done using the solvent system chloroform/ethyl acetais; cyclohexane $\left(32: 9: 1 i^{37}\right.$ and the $k_{F}$ values recorded are after three sevalopments. Visualization was jy a modification of the chlorine/benzidine procedure, ${ }^{33,34}$ substituting o-anisidine for benzidine.

Unless otherwise noted, all reaction were conducter under a nitrogen atmosphere. Evaporations were done on a Berketey rotary evapo:ator after drying over $\mathrm{Na}_{2} \mathrm{SO}_{4}$ : using a water aspirator followed by static evaporation with an oil pump.

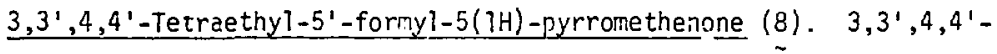
Tetrae $i h v 1-5(1 \mathrm{H})$-pyrromethenone $(7,300 \mathrm{mg}, 1.1 \text { minol })^{22}$ was dissolved in $6 \mathrm{~mL}$ of triflioroacetic acid at room temperature, trimethyl orthoformate ( $3 \mathrm{lnL}, 27 \mathrm{mmol}$ ) was added at once, and the solition was stirred for ive minutes at room temperature. Rapid adjition of $40 \mathrm{~mL}$ of ice water was followed by extraction with $\mathrm{CH}_{2} \mathrm{Cl}_{2}(4 \times 10 \mathrm{~mL})$, and the conbined organic layer was dried and evaporated. The residue was recrystillized from 50 miL of methanol to give $302 \mathrm{mg}(91 \%)$ of 8 as ye: 1 ow needles: mp $194-195^{\circ} \mathrm{C}$; 
UV/vis $\lambda_{\max }\left(\log _{\varepsilon}\right) 415(\mathrm{sh}), 394(3.94), 268(3.94), 261(\mathrm{sh}) ;$ intR $\left(\mathrm{CDCl}_{3}\right) \delta 1.1-1.3\left(4 \times \mathrm{x}, 12 \mathrm{H}, 4 \times \mathrm{CH}_{3}\right), 2.43\left(\mathrm{q}, 2 \mathrm{H}, \mathrm{CH}_{2} \mathrm{CH}_{3}, \mathrm{~J}=7.5 \mathrm{~Hz}\right), 2.57$ $\left(\mathrm{q}, 4 \mathrm{H}, 2 \times \mathrm{CH}_{2} \mathrm{CH}_{3}, \mathrm{~J}=7.5 \mathrm{~Hz}\right), 2.78\left(\mathrm{q}, 2 \mathrm{H}, \mathrm{CH}_{2} \mathrm{CH}_{3}, \mathrm{~J}=7.6 \mathrm{~Hz}\right), 6.10(\mathrm{~s}, \mathrm{lH}$, meso-H), 9.60 ( $\mathrm{s}, \mathrm{H}, \mathrm{CHO}), 10.77(\mathrm{~s}, 1 \mathrm{H}, \mathrm{NH}), 10.85$ ( $\mathrm{s}, \mathrm{HH}, \mathrm{NH})$; Anal. Calcd for $\mathrm{C}_{18} \mathrm{H}_{24} \mathrm{~N}_{2} \mathrm{O}_{2}: C, 72.0 ; \mathrm{H}, 8.0 ; \mathrm{N}, 9.3$. Found: $\mathrm{C}, 72.3 ; \mathrm{H}, 8.0$; $N, 9.4$.

cis-3,4-Dihydro-5'-formyl-3,3',4,4'-tetraethy1-5(1H)-pyrromethenone (9). Pyrromethenone 8 (200 mg, $0.67 \mathrm{mmol})$ was suspended in $50 \mathrm{~mL}$ of methanol, $200 \mathrm{mg}$ of $\tilde{3} \% \mathrm{PdCl}_{2} / \mathrm{SrCO}_{3}{ }^{38}$ was added, and one atmosphere of hydrogen was appljed for four hours, or until the solution turned from yellow to colorless. Filtration and evaporation left a residue which was purified by preparative HPLC: $R_{t}$ (solvent $A, 4.2 \mathrm{~mL} /$ min) $8.4 \mathrm{~min}$, dihydropyrromethanone $28.8 \mathrm{~min}$, pyrromethanone $42.0 \mathrm{~min}$. The product 9 (104 mg, 52\%) was a slightly yellow solid and was recrystallized from methanol: mp $166-170^{\circ} \mathrm{C}$; UV/vis $\lambda_{\max }\left(\log _{\varepsilon}\right) 360(4.10), 242(4.16)$; N N R R $\left(\mathrm{CDCl}_{3}\right) \delta 1.00,1.04,1.11,1.23\left(4 \times t, 12 \mathrm{H}, 4 \times \mathrm{CH}_{3}, 4 \times J=7.6 \mathrm{~Hz}\right), 1.4-1.7$ $\left(\mathrm{m}, 2 \mathrm{H}, \underset{\mathrm{CHCH}_{2}}{\mathrm{CH}_{3}}\right), 1.8-1.95\left(\mathrm{~m}, 2 \mathrm{H}, \mathrm{CHCH}_{2} \mathrm{CH}_{3}\right), 2.47\left(\mathrm{q}, 2 \mathrm{H}, \mathrm{CH}_{2} \mathrm{CH}_{3}\right.$, $\mathrm{J}=7.6 \mathrm{~Hz}), 2.5-2.65(\mathrm{~m}, \mathrm{IH}, 4-\mathrm{H}), 2.73\left(\mathrm{q}, 2 \mathrm{H}, \mathrm{CH}_{2} \mathrm{CH}_{3}, \mathrm{~J}=7.6 \mathrm{~Hz}\right), 2.9-3.0$ $(\mathrm{m}, \mathrm{HH}, 3-\mathrm{H}), 5.38(\mathrm{~s}, \mathrm{lH}$, meso-H), 9.51 (s, $1 \mathrm{H}, \mathrm{CHO})$. Anal. Calcd for $\mathrm{C}_{18} \mathrm{H}_{26} \mathrm{~N}_{2} \mathrm{O}_{2}: \mathrm{C}, 71.5 ; \mathrm{H}, 8.7 ; \mathrm{N}, 9.3$. Found: $\mathrm{C}, 71.6 ; \mathrm{H}, 8.7 ; \mathrm{N}, 9.2$.

trans-3,4-Dihydro-5' -formy $1-3,3^{\prime}, 4^{4}, 4^{\prime}$-tetraethy $1-5(1 \mathrm{H})$-pyrrometinenone (10). The cis compound $g(100 \mathrm{mg}, 0.33 \mathrm{mmol})$ was dissolved in $40 \mathrm{~mL}$ of $1 \mathrm{~N} \mathrm{NaOH}$ in $\mathrm{MeOH}$ and refluxed for $2.5 \mathrm{~h}$. The methanol was evaporated, $20 \mathrm{~mL}$ of $1 \mathrm{M}$ aqueous $\mathrm{H}_{3} \mathrm{PO}_{4}$ was added, the aqlieous solution was extracted with $\mathrm{CH}_{2} \mathrm{Cl}_{2}(4 \times 10 \mathrm{~mL})$, and the combined $\mathrm{CH}_{2} \mathrm{Cl}_{2}$ solution was washed with $10 \mathrm{~mL}$ each of $\mathrm{H}_{2} \mathrm{O}$ and saturated aqueous $\mathrm{NaCl}$ and dried. After filtration and solvent evaporation, the residue was purified by HPLC as described 
for cis compound 9 to give 10 as a slightly yellow solid (76 mg, $76 \%$ ):

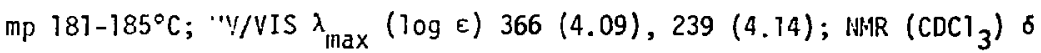
$0.99,1.01,1.11,1.23\left(4 \times t, 12 \mathrm{H}, 4 \mathrm{xCH}_{3}, 4 \times \mathrm{J}=7.5 \mathrm{~Hz}\right), 1.6-1.9(\mathrm{~m}, 4 \mathrm{H}, 2 \mathrm{x}$ $\left.\mathrm{CHCH}_{2} \mathrm{CH}_{3}\right), 2.25-2.35(\mathrm{~m}, \mathrm{IH}, 4-\mathrm{H}), 2.46\left(\mathrm{q}, 2 \mathrm{H}, \mathrm{CH}_{2} \mathrm{CH}_{3}, \mathrm{~J}=7.6 \mathrm{~Hz}\right), 2.73$ (q over $\mathrm{m}, 3 \mathrm{H}, \mathrm{CH}_{2} \mathrm{CH}_{3}$ and $3-\mathrm{H}, \mathrm{J}_{\mathrm{q}}=7.6 \mathrm{~Hz}$ ), 5.32 (s, $\mathrm{lH}$, meso-H), 9.48 $(\mathrm{s}, \mathrm{IH}, \mathrm{CHO}), 10.92(\mathrm{~s}, \mathrm{HH}, \mathrm{NH}), 10.96(\mathrm{~s}, \mathrm{HH}, \mathrm{NH})$; exact mass calcd for $\mathrm{C}_{18} \mathrm{H}_{26} \mathrm{~N}_{2} \mathrm{O}_{2} 302.1995$, found $\underline{\mathrm{m} / \mathrm{e}} 302.1991\left(\mathrm{M}^{+}\right)$.

cis-1,2,3,19,21,24-Hexahydro-2,3,7,8,12,13,17,18-octaethyl-1,19dioxobilin (11). The cis-3,4-dihydro-5'-fomyl-pyrromethenone 9 (6.8 $\mathrm{mg}$, $22 \mu \mathrm{mol})$ and the $5^{\prime}-\mathrm{H}$-pyrrometherone $7(6.1 \mathrm{mg}, 22, \mu \mathrm{mol})$ were dissolved in $1.0 \mathrm{~mL}$ of $\mathrm{CH}_{2} \mathrm{Cl}_{2}, 2,6$-di-t-butyl-4-methy7pyridine ${ }^{39}$ (4.5 mg, 22 umol) and $\mathrm{POBr}_{3}{ }^{40}(2.0 \mathrm{mg}, 7.3 \mu \mathrm{mol})$ were added, and the reaction was left for one hour at room temperature. The reaction then was diluted with $5 \mathrm{~mL}$ of $\mathrm{CH}_{2} \mathrm{Cl}_{2}$, washed successively with $2.5 \mathrm{~mL}$ each of saturated aqueous $\mathrm{NaHCO}_{3}, 1 \mathrm{M}$ aqueous $\mathrm{H}_{3} \mathrm{PO}_{4}, \mathrm{H}_{2} \mathrm{O}$, and saturated aqueous $\mathrm{NaCl}$, dried and evaporated. The residue was purified by HPLC to give $5.7 \mathrm{mg}(46 \%)$ of a blue solid: $R_{t}$ (solvent $\left.8,2.6 \mathrm{~mL} / \mathrm{min}\right) 18 \mathrm{~min}$; UV/Vis $\lambda_{\max }(\log \varepsilon) 580$ $(4.01), 358(4.50), 270(4.26) ; \mathrm{NMR}\left(\mathrm{CDCl}_{3}\right) \delta 1.00\left(t, 3 \mathrm{H}, \mathrm{CH}_{2} \mathrm{CH}_{3}\right.$, $\mathrm{J}=7.5 \mathrm{~Hz}), 1.05-1.3\left(\mathrm{~m}, 21 \mathrm{H}, 7 \times \mathrm{CH}_{2} \mathrm{CH}_{3}\right), 1.41 .8\left(\mathrm{~m}, 4 \mathrm{H}, 2 \times \mathrm{CHCH}_{2} \mathrm{CH}_{3}\right), 2.3-$ $2.7\left(\mathrm{~m}, 13 \mathrm{H}, 6 \mathrm{xCH}_{2} \mathrm{CH}_{3}\right.$ and $\left.4-\mathrm{H}\right), 3.05-3.15(\mathrm{~m}, 1 \mathrm{H}, 3-\mathrm{H}), 5.50,6.01,6.60$ (3xS, 3H, 3x meso-H); NMR (pyridine- $\left.\mathrm{d}_{5}\right) \quad 1.0-1.4\left(\mathrm{~m}, 24 \mathrm{H}, 8 \mathrm{xCH}_{2} \mathrm{CH}_{3}\right)$, $1.6-2.0\left(\mathrm{~m}, 4 \mathrm{H}, 2 \times \mathrm{CHCH}_{2} \mathrm{CH}_{3}\right), 2.3-2.8\left(\mathrm{~m}, 13 \mathrm{H}, 6 \times \mathrm{CH}_{2} \mathrm{CH}_{3}\right.$ and $\left.4-4\right), 3.05-$ $3.10(\mathrm{~m}, 1 \mathrm{H}, 3-\mathrm{H}), 5.50,6.23,6.83(3 \times 5,3 \mathrm{H}, 3 \times$ meso-H) ; exact mass calcd for $\mathrm{C}_{35} \mathrm{H}_{48} \mathrm{~N}_{4} \mathrm{O}_{2} 556.3777$, found $\mathrm{m} / \mathrm{e} 556.3769\left(\mathrm{M}^{+}\right)$. trans-1,2,3,19,21,24-Hexahydro-2,3,7,8,12,13,17,18-octaethy 1-1,19dioxobilin (12). The trans-3,4-dihydro-5'-formylpyrromethenone 10 was coupled and purified as described for 11 . The product was a blue solid 
(37\%): $R_{t}$ (solvent $\left.B, 2.6 \mathrm{~mL} / \mathrm{min}\right) 18 \mathrm{~min} ; U V / V i s \lambda_{\max }(\log \varepsilon) 524$ $(4 . \mathrm{C0}), 354(4.54), 274(4.30) ; \mathrm{NMR}\left(\mathrm{CDCl}_{3}\right) \delta 0.95,1.04(2 \times \mathrm{t}, 6 \mathrm{H}$, $\left.2 \mathrm{CH}_{2} \mathrm{CH}_{3}, 2 \times 3=7.5 \mathrm{~Hz}\right), 1.1-1.3\left(\mathrm{~m}, 18 \mathrm{H}, 6 \mathrm{xCH}_{2} \mathrm{CH}_{3}\right), 1.5-1.9(\mathrm{~m}, 4 \mathrm{H}, 2 \mathrm{x}$ $\left.\mathrm{CHCH}_{2} \mathrm{CH}_{3}\right), 2.2-2.7\left(\mathrm{~m}, 13 \mathrm{H}, 6 \times \mathrm{CH}_{2} \mathrm{CH}_{3}\right.$ and $\left.4-\mathrm{H}\right), 2.80(\mathrm{~m}, 1 \mathrm{H}, 3-\mathrm{H}), 5.47$ (d, $1 \mathrm{H}$, meso-H, $\mathrm{J}=1.2 \mathrm{~Hz}), 6.00,6.58(2 \times \mathrm{S}, 2 \mathrm{H}$, meso-H); NMR (pyridine-

$\left.d_{5}\right) \quad 0.8-1.5\left(m, 24 \mathrm{H}, 8 \times \mathrm{CH}_{2} \mathrm{CH}_{3}\right), 1.6-2.0\left(\mathrm{~m}, 4 \mathrm{H}, 2 \times \mathrm{CHCH}_{2} \mathrm{CH}_{3}\right), 2.3-2.7$ $\left(\mathrm{m}, 13 \mathrm{H}, 6 \times \mathrm{CH}_{2} \mathrm{CH}_{3}\right.$ and $\left.4-\mathrm{H}\right), 2.75-2.83(\mathrm{~m}, 1 \mathrm{H}, 3-\mathrm{H}), 5.50,6.23,6.83$ $\left(3 \times 5,3 \mathrm{H}\right.$, meso-H); exact mass calcd for $\mathrm{C}_{35} \mathrm{H}_{48} \mathrm{~N}_{4} \mathrm{O}_{2} 556.3777$, found $\mathrm{m} / \mathrm{e}$ $556.3795\left(M^{+}\right)$.

4-Ethyl-3-methyl-3-pyrrolin-2-one (13a) ${ }^{27}$ The cyanohydrin of ethyl 2-methyl-3-oxopentanoate ${ }^{41}$ was prepared through the bisulfite adduct ${ }^{28}$ without purification and carried on directly to the pyrrolinone 13a: mp $79-81^{\circ} \mathrm{C}\left(1 \mathrm{it}{ }^{27} \mathrm{mp} 81-83^{\circ} \mathrm{C}\right)$; NMR $\left(\mathrm{COCl}_{3}\right) \& 1.10\left(\mathrm{t}, 3 \mathrm{H}, 4-\mathrm{CH}_{2} \mathrm{CH}_{3}\right.$, $\mathrm{J}=7.5 \mathrm{~Hz}), 1.77\left(\mathrm{~s}, 3 \mathrm{H}, 3-\mathrm{CH}_{3}\right), 2.38\left(q, 2 \mathrm{H}, 4-\mathrm{CH}_{2} \mathrm{CH}_{3}, \mathrm{~J}=7.5 \mathrm{~Hz}\right), 3.82$ $\left(\mathrm{s}, 2 \mathrm{H}, 5-\mathrm{CH}_{2}\right), 7.68$ (br s, $\left.\mathrm{iH}, \mathrm{NH}\right)$.

3-Ethyl-4-methyl-3-pyrrol in-2-one (13b). ${ }^{23}$ Ethyl 2-ethyl-3-oxobutanoate ${ }^{42}$ was converted as above to pyrrolinone $13 \mathrm{~b}:{ }^{26}$ mp $101-102^{\circ} \mathrm{C}$ (1it. $\left.{ }^{23} \mathrm{mp} 101^{\circ} \mathrm{C}\right)$; NMR $\left(\mathrm{CDCl}_{3}\right) \& 1.06\left(\mathrm{t}, 3 \mathrm{H}, 3-\mathrm{CH}_{2} \mathrm{CH}_{3}, \mathrm{~J}=7.5 \mathrm{~Hz}\right), 1.97$ $\left(\mathrm{s}, 3 \mathrm{H}, 4-\mathrm{CH}_{3}\right), 2.25\left(\mathrm{q}, 2 \mathrm{H}, 3-\mathrm{CH}_{2} \mathrm{CH}_{3}, \mathrm{~J}=7.5 \mathrm{~Hz}\right), 3.80\left(\mathrm{~s}, 2 \mathrm{H}, 5-\mathrm{CH}_{2}\right), 8.14$ (br s, IH, NH).

\section{t-Buty 1-3', 4-Dimethyl-3-ethyl-4' - (2-methoxycarbonylethy 1$)-5$ (1H)-} pyrromethenone-5'-carboxylate $(15 \mathrm{a})$. To a solution of $0.692 \mathrm{~g}(2.36 \mathrm{mmol})$ of t-butyl 5-formyl-3-(2-methoxycarbonylethyl)-4-methylpyrrole-2-carboxylate $(14)^{30}$ and $0.433 \mathrm{~g}$ (3.47 mmol) of 4-ethyl-3-methylpyrrol in-2-one (13a) in $1.3 \mathrm{~mL}$ of $\mathrm{MeOH}$ was added $1.71 \mathrm{~g}$ (30.5 mmol) $\mathrm{KOH}$ in $6.5 \mathrm{~mL}$ of $\mathrm{H}_{2} \mathrm{O}$ at room temperature. The reaction was stirred for $18 \mathrm{~h}$, then diluted with $26 \mathrm{~mL}$ of $\mathrm{H}_{2} \mathrm{O}$ and $\mathrm{SO}_{2}$ (g) was passed through until the $\mathrm{pH}$ registered 3 . 
The precipitate formed during the acidification was filtered, wasted with $10 \mathrm{mil}$ aqueous TFA, and dried to give $0.870 \mathrm{~g}(95 \%)$ of the $4^{\prime}$-propionic acid-pyrromethenone derivative. This acid was directly esterified by adding $1.2 \mathrm{~mL}$ of $\mathrm{MeOH}$ and then $23.6 \mathrm{~mL}$ of $0.1 \mathrm{M} \mathrm{CH}_{2} \mathrm{~N}_{2}$ in ether ${ }^{43}$ and stirring for one hour at room temperature. The heterogeneous reaction was filtered and the solid recrystallized from MeOH/ether/hexane to give $3.10 \mathrm{~g}(86 \%)$ of a yellow solid. The filtrate was evaporated and the residue was recrystallized from the same solvent to give an additional $0.26 \mathrm{~g}$ of product for a total yield of $15 \mathrm{a}$ of $93 \%$ : $\mathrm{mp} 188-190^{\circ} \mathrm{C}$ (dec); UV/VIS $\lambda_{\max }(\log \varepsilon) 400(\mathrm{sh}), 380(4.38), 257(4.32), 250(\mathrm{sh}) ; \operatorname{NMR}\left(\mathrm{CDCl}_{3}\right)$ $\delta 1.38\left(\mathrm{t}, 3 \mathrm{H}, 3-\mathrm{CH}_{2} \mathrm{CH}_{-3}, \mathrm{~J}=7.5 \mathrm{~Hz}\right), 1.56\left(\mathrm{~s}, 9 \mathrm{H}, \mathrm{C}\left(\mathrm{CH}_{3}\right)_{3}\right), 1.97(\mathrm{~s}, 3 \mathrm{H}$, 4- $\left.\mathrm{CH}_{3}\right), 2.10\left(\mathrm{~s}, 3 \mathrm{H}, 3^{\prime}-\mathrm{CH}_{3}\right), 2.45-2.65\left(\mathrm{~m}, 4 \mathrm{H}, 3-\mathrm{CH}_{2} \mathrm{CH}_{3}\right.$ overlapping $4^{\prime}-\mathrm{CH}_{2} \mathrm{CH}_{2} \mathrm{CO}_{2} \mathrm{CH}_{3}$ ), 3.01 ( $\left.\mathrm{t}, 2 \mathrm{H}, 4^{\prime}-\mathrm{CH}_{2} \mathrm{CH}_{2} \mathrm{CO}_{2} \mathrm{CH}_{3}, \mathrm{~J}=7.5 \mathrm{~Hz}\right), 3.68(\mathrm{~s}, 3 \mathrm{H}$, $\left.\mathrm{OCH}_{3}\right), 5.98\left(\mathrm{~s}, 1 \mathrm{H}\right.$, meso-H) : exact mass calcd for $\mathrm{C}_{22} \mathrm{H}_{30} \mathrm{H}_{2} \mathrm{O}_{5} 402.2155$, found $\mathrm{m} / \mathrm{e} 402.2158\left(\mathrm{MH}^{+}\right)$.

t-Eutyl 3,3'-Dimethyl-4-ethy]-4'-(2-methoxycarbonylethy]) -5(1H)pyrromethenone-5'-carboxylate $(15 \mathrm{~b}) .^{30}$ This compound was prepared as described. 30 The filtrate was evaporated and the residue was recrystallized from $\mathrm{MeOH} / \mathrm{ether} /$ hexane to give $15 \mathrm{~b}$ in a total yield of $92 \%$ : mp $205-207^{\circ} \mathrm{C}$ ( $7 \mathrm{it.}^{30} \mathrm{mp} 206-208^{\circ} \mathrm{C}$ ); exact mass calcd for $\mathrm{C}_{22} \mathrm{H}_{30} \mathrm{~N}_{2} \mathrm{O}_{5} 402.2155$, found in/e $402.2141\left(\mathrm{M}^{+}\right)$.

3',4-Dimethyl-3-ethyl-.4' -(2-me thoxycarkonylethy 1) -5(1H) -pyrromethenone $(17 a) .44$ The $5{ }^{\prime}$-t-butyioxycarbony $1 p_{j}$ rromethenone $\underset{\sim \sim}{15 a}(0.50 \mathrm{~g}, 1.2 \mathrm{mmol})$ was added to $20 \mathrm{~mL}$ of anhydrous trifluoroacetic acid and stirred for two hours at room temperature. The sclution was poured into $200 \mathrm{~mL}$ of water and filtered, and the precipitate was washed with water and died to give

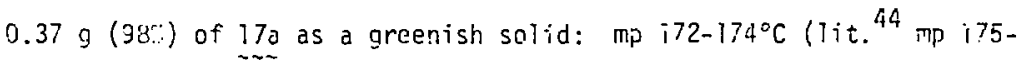


$\left.776^{\circ} \mathrm{C}\right)$; UV/vis $\lambda_{\max }(\log \varepsilon) 395(5.32), 261$ (4.83), $231(4.77) ; \mathrm{MHR}\left(\mathrm{CDCl}_{3}\right)$

$\left.\delta 1.189 \mathrm{t}, 3 \mathrm{H}, 3-\mathrm{CH}_{2} \mathrm{CH}_{3}, \mathrm{~J}=7.6 \mathrm{~Hz}\right), 1.94\left(\mathrm{~s}, 3 \mathrm{H}, 4-\mathrm{CH}_{3}\right), 2.16(\mathrm{~s}, 3 \mathrm{H}$, $\left.3^{\prime}-\mathrm{CH}_{3}\right), 2.5-2.65\left(\mathrm{~m}, 4 \mathrm{H}, 3-\mathrm{CH}_{2} \mathrm{CH}_{3}\right.$ overlapping $\left.4^{\prime}-\mathrm{CH}_{2} \mathrm{CH}_{2} \mathrm{CO}_{2} \mathrm{CH}_{3}\right), 2.77$ ( $t$, $2 \mathrm{H}, 4^{\prime}-\mathrm{CH}_{2} \mathrm{CH}_{2} \mathrm{CO}_{2} \mathrm{CH}_{3}, \mathrm{~J}=7.5 \mathrm{~Hz}$ ), $3.699 \mathrm{~s}, 3 \mathrm{H}, \mathrm{OCH}_{3}$ ), 6.33 (s, $7 \mathrm{H}$, meso-H), $6.89\left(\mathrm{~d}, 1 \mathrm{H}, 5^{\prime}-\mathrm{H}, \mathrm{J}=2.7 \mathrm{~Hz}\right.$ ), 10.05 (br s, $\left.1 \mathrm{H}, \mathrm{NH}\right), 11.63$ (br s, $1 \mathrm{H}, \mathrm{NH}$ ).

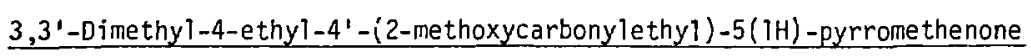
(17b). ${ }^{44}$ The 5'-t-butyloxycarbonylpyrromethenone ${ }_{\sim \sim}^{15 b}$ was converted to the $5^{\prime}-\mathrm{H}$ compnund as described above in $99 \%$ yield: $\mathrm{mp} 200-202^{\circ} \mathrm{C}$ (1it. ${ }^{44}$ mp 203.5-205 $\mathrm{C})$; UV/vis $\lambda_{\max }(\log$ E) $394(5.30), 259$ (4.80), 230 (4.74); NMR $\left(\mathrm{CDCl}_{3}\right) \quad 1.15\left(\mathrm{t}, 3 \mathrm{H}, 4-\mathrm{CH}_{2} \mathrm{CH}_{3}, \mathrm{j}=7.5 \mathrm{~Hz}\right), 2.13\left(\mathrm{~s}, 6 \mathrm{H}, 3-\mathrm{CH}_{3}\right.$ and $\left.3^{\prime}-\mathrm{CH}_{3}\right), 2.41\left(\mathrm{q}, 2 \mathrm{H}, 4-\mathrm{CH}_{2} \mathrm{CH}_{3}, \mathrm{~J}=7.5 \mathrm{~Hz}\right), 2.60\left(\mathrm{t}, 2 \mathrm{H}, 4^{\prime}-\mathrm{CH}_{2} \mathrm{CH}_{2} \mathrm{CO}_{2} \mathrm{CH}_{3}\right.$, $\mathrm{J}=7.5 \mathrm{~Hz}), 2.79\left(\mathrm{t}, 2 \mathrm{H}, 4^{\prime}-\mathrm{CH}_{2} \mathrm{CH}_{2} \mathrm{CO}_{2} \mathrm{CH}_{3}, \mathrm{~J}=7.5 \mathrm{~Hz}\right), 3.70\left(\mathrm{~s}, 3 \mathrm{H}, \mathrm{OCH}_{3}\right)$, $6.13\left(\mathrm{~s}, \mathrm{IH}\right.$, meso-H) $6.78\left(\mathrm{~d}, \mathrm{TH}, 5^{\prime}-\mathrm{H}, \mathrm{J}=2.8 \mathrm{~Hz}\right)$.

3',4-Dimethy]-3-ethyl-5'-formy $1-4^{\prime}$ - (2-methoxycarbonylethy 1$)-5(1 \mathrm{H})$ pyrromethenone $(16 \mathrm{a}) .{ }^{45,46}$ The $5^{\prime}$-t-t-butyloxycarbonylpyiromethencne $15 \mathrm{a}$ $(0.465 \mathrm{~g}, 1.16 \mathrm{mmol})$ was added to $2.3 \mathrm{~mL}$ of anhydrous trifluoroacetic acid and stirred for $2.5 \mathrm{~h}$ at room temperature. Trimethyl orthoformate $(0.70 \mathrm{~mL}, 6.4 \mathrm{mmol})$ was added all at once and, after five minutes of stirring, the reaction was quenched by pouring it into $23 \mathrm{~mL}$ of water. The aquesus phase was extracted with $25 \%(v / v)$ isopropyl alcohol in chloroform $(6 \times 10 \mathrm{~mL})$ and the combined organic phases were washed with 20 $\mathrm{mL}$ of water and $20 \mathrm{~mL}$ of saturated aqueous sodium chloride and dried. Evaporation and recrystallization of the residue from methanol/hexane gave $0.310 \mathrm{~g}(81 \%)$ of ?oa as a yellow solid: mp $207-209^{\circ} \mathrm{C}$ (1it. ${ }^{45} \mathrm{mp}$ $\left.205.5^{\circ} \mathrm{C}, 1 \mathrm{it} .{ }^{46} \mathrm{mp} 2 \mathrm{I}^{\circ} \mathrm{C}\right)$; NMR $\left(\mathrm{CDCl}_{3}\right) \delta 1.20\left(\mathrm{t}, 3 \mathrm{H}, 3 \mathrm{CH}_{2} \mathrm{CH}_{3}, \mathrm{~J}=7.6 \mathrm{~Hz}\right)$, $2.00\left(\mathrm{~s}, 3 \mathrm{H}, 4-\mathrm{CH}_{3}\right), 2.14\left(\mathrm{~s}, 3 \mathrm{H}, 3^{\prime}-\mathrm{CH}_{3}\right), 2.55\left(q, 2 \mathrm{H}, 3-\mathrm{CH}_{2} \mathrm{CH}_{3}, \mathrm{~J}=7.6 \mathrm{~Hz}\right)$, $2.61\left(t, 2 \mathrm{H}, 4^{\prime}-\mathrm{CH}_{2} \mathrm{CH}_{2} \mathrm{CO}_{2} \mathrm{CH}_{3}, J=7.7 \mathrm{~Hz}\right), 3.09\left(\mathrm{t}, 2 \mathrm{H}, 4^{\prime}-\mathrm{CH}_{2} \mathrm{CH}_{2} \mathrm{CO}_{2} \mathrm{CH}_{3}\right.$, 
$\mathrm{J}=7.7 \mathrm{~Hz}), 3.68\left(\mathrm{~s}, 3 \mathrm{H}, \mathrm{OCH}_{3}\right), 5.99(\mathrm{~s}, \mathrm{HH}$, meso-H), $9.77(\mathrm{~s}, \mathrm{IH}, \mathrm{CHO})$, 10.69 (br s, $1 \mathrm{H}, \mathrm{NH}$ ), 10.90 (br s, $1 \mathrm{H}, \mathrm{NH}$ ); exact mass calcd for $6_{18} \mathrm{H}_{22} \mathrm{~N}_{2} \mathrm{O}_{4}$ 330.1580 , found $\mathrm{m} / \mathrm{e} 330.1583\left(\mathrm{M}^{+}\right)$.

3,3'-Dimethyl-4-ethyl-5'-formyl-4' -(2-methoxycarbonylethyl) -5(1H)pyrromethenone (16b). $9,45,46$ The cbove procedure was used on the 5 '-t-butyloxycarbonylpyrromethenone $\underset{\sim \sim}{15 b}$ to give a $76 \%$ yield of $\underset{\sim \sim \sim}{16 b}$ as a yellow solid: mp $201-203^{\circ} \mathrm{C}\left(1 \mathrm{it.}^{9} \mathrm{mp} 202^{\circ} \mathrm{C}\right.$, lit. ${ }^{45} \mathrm{mp} 218-220^{\circ} \mathrm{C}$, lit. ${ }^{46}$ mp 212 $\left.{ }^{\circ} \mathrm{C}\right)$; NMR $\left(\mathrm{CDCl}_{3}\right) \delta 1.12\left(t, 3 \mathrm{H}, 4-\mathrm{CH}_{2} \mathrm{CH}_{3}, \mathrm{~J}=7 \mathrm{~Hz}\right), 2.13\left(\mathrm{~s}, 6 \mathrm{H}, 3-\mathrm{CH}_{3}\right.$ and $\left.3^{\prime}-\mathrm{CH}_{3}\right), 2.45\left(9,2 \mathrm{H}, 4-\mathrm{CH}_{2} \mathrm{CH}_{3}, \mathrm{~J}=7.5 \mathrm{~Hz}\right), 2.60\left(t, 2 \mathrm{H}, 4^{\prime}-\mathrm{CH}_{2} \mathrm{CH}_{2} \mathrm{CO}_{2} \mathrm{CH}_{3}\right.$, $\mathrm{J}=7.6 \mathrm{~Hz}$ ), $3.08\left(\mathrm{t}, 2 \mathrm{H}, 4^{\prime}-\mathrm{CH}_{2} \mathrm{CH}_{2} \mathrm{CO}_{2} \mathrm{CH}_{3}, \mathrm{~J}=7.6 \mathrm{~Hz}\right), 3.67\left(\mathrm{~s}, 3 \mathrm{H}, \mathrm{OCH}_{3}\right), 5.96$ (s, $1 \mathrm{H}$, meso-H), $9.74(\mathrm{~s}, \mathrm{IH}, \mathrm{CHO}), 10.58$ (br s, $1 \mathrm{H}, \mathrm{NH}), 10.78$ (br s, $1 \mathrm{H}, \mathrm{NH})$; exact mass calcd for $\mathrm{C}_{18} \mathrm{H}_{22} \mathrm{~N}_{2} \mathrm{O}_{4} 330.1580$, found $\mathrm{m} / \mathrm{e} 330.1576\left(\mathrm{M}^{+}\right)$. cis-3,4-Dihydro-3', 4-dimethyl-3-ethy $1-5^{\prime}$-formy $1-4^{\prime}-$ (2-methoxycarbonyl ethyl)-5(1H)-pyrromethenone (18a)..$^{25,26}$ The 5'-fomylpyrromethenone $16 a$ was reduced as described for the tetraethylpyrromethenone 8 above. The product was purified by HPLC to give $\underset{\sim \sim}{18}$ as a slightly yellow solid in $37 \%$ yield: $R_{t}$ (solvent $C, 2.0 \mathrm{~mL} / \mathrm{min}$ ) $34.8 \mathrm{~min}$ (the corresponding pyrromethanone and dihydropyrromethanone were not eluted under these conditions); UV/Vis $\lambda_{\max }(\log \varepsilon) 350(4.11), 240(4.10)$. The NMR spectral assignments in $\mathrm{CDCl}_{3}$ are found in Table I and the 3-H/4-H coupling constant is found in Table IV. Exact mass calcd for $\mathrm{C}_{18} \mathrm{H}_{24} \mathrm{~N}_{2} \mathrm{O}_{4} 332.1736$, found m/e $332.1731\left(M^{+}\right)$.

cis -3,4-Dihydro-3,3'-dimethyl-4-ethyl-5'-formy l-4'-(2-methoxycarbonylethyl)-5(1H)-pyrrometherione (18b).25,26 The above procedure was carried out on pyrromethenone $\underset{\sim}{16 \mathrm{~b}}$ to give a $44 \%$ yield of $18 \mathrm{~b}$ after preparative HPLC purification: $R_{t}$ (solvent $C, 2.0 \mathrm{~mL} / \mathrm{min}$ ) $34.8 \mathrm{~min}$ (the corresponding pyrrcmethanone and dihydropyrromethanone were not eluted under these 
conditions); UV/Vis $\lambda_{\max }(\log \varepsilon) 354$ (4.12), 241 (4.11). The lith spectrat assignments are found in Table I and the 3-H/4-H coupting constant is found in Table IV. Exact mass calcd for $\mathrm{C}_{18} \mathrm{H}_{24} \mathrm{~N}_{2} \mathrm{O}_{4} 332.1736$, found m/e $332.1738\left(M^{+}\right)$.

trans-3,4-Dihydro-3',4-dimethyl-3-ethyl-5'-formy 1-4'-(2-methoxycarbony 1ethyl)-5(1H)-pyrromethenone $(19 a) .^{25,26}$ The corresponding cis-dihydropyrromethenone $18 \mathrm{a}(0.100 \mathrm{~g}, 0.30 \mathrm{mmol})$ was heated under reflux in $30 \mathrm{~mL}$ of $0.5 \mathrm{M}$ sodium methoxide in methanol for $2.5 \mathrm{~h}$ then cooled, and $15 \mathrm{~mL}$ of 1. OM aqueous $\mathrm{KH}_{2} \mathrm{PO}_{4}$ was added and the methanol was evaporated. The residue was extracted with $25 \%(\mathrm{v} / \mathrm{v})$ isopropyl alcohol in methanol $(6 \times 15 \mathrm{~mL})$ and the combined organic phase was washed with $15 \mathrm{~mL}$ of water and $15 \mathrm{~mL}$ of saturated aqueous $\mathrm{NaCl}$ and dried. Evaporation left a residue which was purified by preparative HPLC to give 19a as a slightly yellow solid in $48 \%$ yield $(48 \mathrm{mg}): \quad R_{t}$ (solvent $C, 2.0 \mathrm{~mL} / \mathrm{min}$ ) 37.3 min (the corresponding pyrromethenone was not eluted under these conditions);

UV/vis $\lambda_{\max }\left(\log _{\varepsilon}\right) 351$ (4.11), 242 (4.09). The NMR spectral assignmants in $\mathrm{CDCl}_{3}$ are found in Table I and the $3-\mathrm{H} / 4-\mathrm{H}$ coupling constant is found in Table IV. Exact mass calcd for $\mathrm{C}_{18} \mathrm{H}_{24} \mathrm{~N}_{2} \mathrm{O}_{4} 332.1736$, found m/e $332.1727\left(M^{+}\right)$.

trans -3,4-0 ihydro-3,3'-dimethyl-4-ethy?-5' - formyl-4'-(2-me thaxycarbonylethyl) $-5(1 \mathrm{H})$-pyrromethenone $(19 \mathrm{~b}) .^{25,26}$ The procedure as described for $19 \mathrm{a}$ above was used to give a $45 \%$ yield of $19 \mathrm{~b}$ : $i_{t}$ (solvent $c, 2.0 \mathrm{~mL} / \mathrm{mm}$ ), $31.3 \mathrm{~min}$ (the corresponding pyrromethanone was not eluted under these conditions); UV/vis $\lambda_{\max }\left(\log _{\varepsilon}\right) 353(4.13), 241$ (4.10). The NMR spectral assignments in $\mathrm{COCl}_{3}$ are found in Table I and the 3-H/4-H coupling constant is found in Table IV. Exact mass calcd for $\mathrm{C}_{18} \mathrm{H}_{24} \mathrm{~N}_{2} \mathrm{O}_{4} 332.1736$, found $\mathrm{nl} / \mathrm{e} 332.1726\left(\mathrm{H}^{+}\right)$. 
cis-3,18-Diethyl-1,2,3,19,21,24-hexahydro-2,7,73,17-tetramethyl-1,19dioxobilin-8,12-dipropionic acid Methyl Ester $(\underset{\sim}{20 a}) .25$ This compound

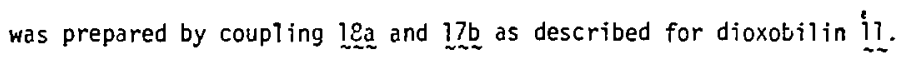
The product was purified by preparative HPLC to give $41 \%$ of $20 \mathrm{a}$ as a blue solid: $R_{t}$ (solvent $D, 2.0 \mathrm{~mL} / \mathrm{min}$ ) $30 \mathrm{~min}$. NHAR spectral properties are found in Tables II, III, and IV. Exact mass calcd for $\mathrm{C}_{35} \mathrm{H}_{44} \mathrm{~N}_{4} \mathrm{O}_{6}$ 616.3261, found $\underline{\mathrm{m} / \mathrm{e}} 616.3270\left(\mathrm{M}^{+}\right)$.

cis-2,17-Diethyl-1,2,3,19,21,24-hexahydro-3,7,13,18-tetramethyl-1,19dioxobilin-8,12-dipropion:c Acid Methyl Ester $(20 \mathrm{~b})^{25}$ This compound was prepared by coupling $\underset{\sim}{18 b}$ and $\underset{\sim}{17 a}$ as described for dioxobilin 11. The product was purified by preparative HPLC to give $45 \%$ of $\underset{\sim}{20 \mathrm{~b}}$ as a blue solid: $R_{t}$ (solvent $D, 2.0 \mathrm{~mL} / \mathrm{min}$ ) $32 \mathrm{~min}$. NMR spectral properties are found in Tables II, III, and IV. Exact mass caled for $\mathrm{C}_{35} \mathrm{H}_{44} \mathrm{~N}_{4} \mathrm{O}_{6} 616.3261$, found $\mathrm{m} / \mathrm{e} 616.3236\left(\mathrm{M}^{+}\right)$.

trans-3,18-Diethyl-1,2,3,19,21,24-hexahydro-2,7,13,17--tetremethyl-1,19dioxobilin-8,12-dipropionic Acid Methyl Ester (21a) ${ }^{26}$ This compound was prepared by coupling 19a with 17b as described for dioxobilin 11 . The preduct was purified by preparative HPLC to give $58 \%$ of 21 a as a b7ue solid: $R_{t}$ (solvent $\left.D, 2.0 \mathrm{~mL} / \mathrm{min}\right) 25 \mathrm{~min}$; UV/vis $\lambda_{\max }(\log \varepsilon)$ $583(4.06), 343(4.45), 272(4.25)$. NMR spectral properties are found in Tables II, III, and IV. Exact mass caicd for $\mathrm{C}_{35} \mathrm{H}_{44} \mathrm{~N}_{4} \mathrm{O}_{6} 616.3267$, found IIIe $616.3233\left(\mathrm{M}^{+}\right)$.

trans-2.17-Diethy $1-1,2,3,19,21,24$-hexahydro-3,7,13,18-teträmethyl-1,19dioxobilin-8,12-dipropionic Acid Methyl Ester (21b) 25,26 This compound was prepared by coupling 196 with 17 a as described for dioxobilin 11. The product was purified by preparative HPLC to give $36 \%$ of $2 \mathrm{Jb}$ as a blue solid: $n_{t}$ (solvent $0,2.0 \mathrm{~mL} / \mathrm{min}$ ) $25 \mathrm{~min}$. NMR spectral properties are 
found in Tables II, III, and IV. Exact mass calcd for $\mathrm{C}_{35} \mathrm{H}_{44} \mathrm{~N}_{4} \mathrm{O}_{6}$ 616.3261, found $\mathrm{m} / \mathrm{e} 616.3247\left(\mathrm{H}^{+}\right)$.

3,4-Diethyl-?H-pyrrole-2,5-dione(3,4-Diethylmaleimide). 47,48 3,4 -Diethylpyrrole $20,21,49(10 \mathrm{~g}, 81 \mathrm{mmol})$ in $15 \mathrm{~mL}$ of pyridine containing $12 \mathrm{~mL}$ of $30 \%$ aqueous $\mathrm{H}_{2} \mathrm{O}_{2}$ was heated at reflux for five minutes. Another $3 \mathrm{~mL}$ of $\mathrm{H}_{2} \mathrm{O}_{2}$ was added and heating continued for ten more minutes. 22 The solvent was eyaporated and the residue was taken up in $100 \mathrm{~mL}$ of $\mathrm{CHCl}_{3}$. The organic phase was washed with $30 \mathrm{~mL}$ of $\mathrm{IM} \mathrm{NaOH}$, dried, and cautiously evaporated, leaving the crude pyrrolinone $(10.0 \mathrm{~g}, 97 \%)$. This crude product was dissolved in $175 \mathrm{~mL}$ of acetone, a solution of $17.1 \mathrm{~g}$ (171 mmol) of $\mathrm{CrO}_{3}$ in $170 \mathrm{~mL}$ of $2 \mathrm{M}_{2} \mathrm{HO}_{4}$ was added, and the reaction was heated at $50^{\circ} \mathrm{C}$ for one hour. After cooling the solution to room temperature, the acetone was evaporated and the aqueous residue was extracted with EtOAC $(7 \times 100 \mathrm{~mL})$. The combined organic phase was washed with $100 \mathrm{~mL}$ of saturated aqueous $\mathrm{NaCl}$, dried, and evaporated, and the residue was purified by silica gel chromatography using isooctane/ether, $1 / 1$. to give $5.8 \mathrm{~g}(47 \%)$ of 3,4-diethylmaleimide: mp $69-70^{\circ} \mathrm{C}\left(1 \mathrm{it.}^{47} \mathrm{mp} 68^{\circ} \mathrm{C}\right.$, 1it. $\left.{ }^{48} \mathrm{mp} 68-70^{\circ} \mathrm{C}\right) ; \mathrm{R}_{\mathrm{t}}(\mathrm{GC}) 3.30 \mathrm{~min}$.

cis-3,4-Diethylpyrrolidine-2,5-dione(cis-3,4-Diethyl succinimide).

To $180 \mathrm{mg}(0.85 \mathrm{mmol})$ of the above 3,4-diethyl-iH-pyrrole-2,5-dione in $10 \mathrm{~mL}$ of ethyl acetate was added $18 \mathrm{mg} \mathrm{P_{2 }}$. Hydrogen at 50 psi was applied for 6 hours, 31 the solution was filtered through celite, and the filtrate was evaporated to give $132 \mathrm{mg}(100 \%)$ of residlie. GC analysis showed a 95/5 mixture of cis/trans isomers which may be due to epimerization during analysis, as the cis compound was never found completely free of the trans by GC analysis. ${ }^{50}$ The product was recrystallized from $\mathrm{MeOH} / \mathrm{H}_{2} \mathrm{O}$ and sublimed at $70^{\circ} \mathrm{C} / 1$ torr to give a $98 / 2$ ratio of cis/trans 3,4-diethyl- 
succinimide as white crysta]s: $\ln 37-39^{\circ} \mathrm{C} ; R_{t}(G C)$ cis $5.16 \mathrm{~min}$, trans 4.17 min; UV $\lambda_{\text {max }}(\log$ E) $246(1.93), 221$ (2.28); IR (KBr) 3130, $1670,1340 \mathrm{~cm}^{-1}$; NMR $\left(\mathrm{CDCl}_{3}, 90 \mathrm{HHz}\right) \delta 1.02\left(t, 6 \mathrm{H}, 2 \mathrm{CCH}_{2} \mathrm{CH}_{3}, \mathrm{~J}=7.5 \mathrm{~Hz}\right)$, 1.54-1.77 (m, 4H, $\left.2 \times \mathrm{CH}_{2} \mathrm{CH}_{3}\right), 2.70-2.83(\mathrm{~m}, 2 \mathrm{H}, 2 \times \mathrm{CH}), 8.32$ (br s, $1 \mathrm{H}$, NH). Anal. Calcd for $\mathrm{C}_{8} \mathrm{H}_{13} \mathrm{HO}_{2}$ : C, 67.9; $\mathrm{H}, 8.4 ; \mathrm{N}, 9.0$. Found: C, $61.9 ; H, 8.4 ; N, 8.8$.

trans-3,4-Diethylpyrrol idine-2,5-dione(trans-3,4-Diethyl succinimide). To the above cis-3,4-diethylpyrrolidirie-2,5-dione (153 $\mathrm{mg}, 1.00$ mal) was added a solution of $2.24 \mathrm{~g}(2.00 \mathrm{mmol})$ of potassium t-butoxide in $60 \mathrm{~mL}$ of t-butanol. After refluxing for two hours, the solution was cooled and the t-butyl alcohol was evaporated. Aqueous $\mathrm{IM}_{3} \mathrm{PO}_{4}(20 \mathrm{~mL})$ was added and the aqueous phase was extracted with $\mathrm{CH}_{2} \mathrm{Cl}_{2}(5 \times 10 \mathrm{~mL})$ which was dried and eyaporated to give $143 \mathrm{mg}\left(93_{\%}^{\circ}\right)$ of trans-3,4-diethylsuccinimide, mp $59-60^{\circ} \mathrm{C}$ $R_{t}$ (GC) $4.17 \mathrm{~min} ;$ UV $\lambda_{\max }(\log E) 244(1.64), 220$ (2.22); IR (KBr) $3140,1670,1175 \mathrm{~cm}^{-1}$; $\mathrm{NMR}\left(\mathrm{CDCl}_{3}, 90 \mathrm{MHz}\right) \delta 1.00\left(t, 6 \mathrm{H}, 2 \mathrm{xCH}_{2} \mathrm{CH}_{3}\right.$, $3=7.5 \mathrm{~Hz}), 1.60-1.90\left(\mathrm{~m}, 4 \mathrm{H}, 2 \times \mathrm{CH}_{2} \mathrm{CH}_{3}\right), 2.42-2.53(\mathrm{~m}, 2 \mathrm{H}, 2 \times \mathrm{CH}), 8.80$ (br s, $1 \mathrm{H}, \mathrm{NH}$ ). Anal. Calcd for $\mathrm{C}_{8} \mathrm{H}_{13} \mathrm{HO}_{2}: \mathrm{C}, 61.9 ; \mathrm{H}, 8.4 ; \mathrm{N}, 9.0$. Found: $\mathrm{C}, 62.2 ; \mathrm{H}, 8.4 ; \mathrm{N}, 8.9$.

3-Ethyl-4-methyl-1H-pyrrole-2,5-dione(3-Ethyl-4-methylma? eimide).$^{15,47,51}$ This compound was prepared from 3-ethyl-4-nethylpyrrole $20,21,52$ as described for the 3,4-diethyl-1H-pyrrole-2,5-dione above in 45\% yield: mp 66-68 ${ }^{\circ} \mathrm{C}$ (1it. ${ }^{15} \mathrm{mp} 66-67^{\circ} \mathrm{C}$, lit. $^{47} \mathrm{mp} 67-68^{\circ} \mathrm{C}$, iit. ${ }^{51} \mathrm{mp} 68^{\circ} \mathrm{C}$ ); $\mathrm{R}_{f}$ (TLC) 0.89 . cis-3-Ethyl-4-methylpyrrolidine-2,5-dione(cis-3-Ethyl-4-methyl${ }_{\text {succinimide). }}^{15,31}$ This compound was prepared as described ${ }^{15}$ in quantitative yield: $m p 43-50^{\circ} \mathrm{C}\left(1 \mathrm{it} . .^{31} \mathrm{mp} 50^{\circ} \mathrm{C}\right) ; R_{f}$ (TLC) cis 0.20 , trans $0.30 ; R_{t}(G C)$ cis $1.9 \mathrm{~min}$, trans 1.3 min. ${ }^{50}$

trans-3-E thyl-4-metizyl pyrrol idine-2.5-dione (trans-2-Ethyl--4-methyl- 
succinimide). ${ }^{31}$ This compound was prepared in an analogous manner to the trans-3,4-diethyl-pyrrolidine-2,5-dione above in $64 \%$ yield. mp $57-59^{\circ} \mathrm{C}$

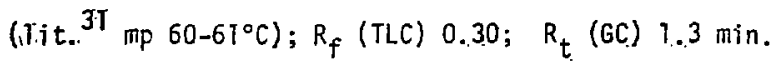

BiTe Pigment Oxidative Degradations. ${ }^{32}$ To a solution of $250 \mathrm{mg}$ of $\mathrm{Na}_{2} \mathrm{Cr}_{2} \mathrm{O}_{7}$ in $6 \mathrm{~mL}$ of $\mathrm{H}_{2} \mathrm{O}$ was added $10 \mathrm{mg}$ of the bile pigment in $2 \mathrm{~mL}$ of THF. The oxidation was allowed to proceed at room temperature for $2 \mathrm{~h}$, then $10 \mathrm{~mL}$ of $\mathrm{H}_{2} \mathrm{O}$ was added and the mixture was extracted with $\mathrm{CH}_{2} \mathrm{Cl}_{2}$. $(5 \times 10 \mathrm{~mL})$. The combined organic phase was dried and evaporated. The residue of oxidation products from the octaethyldioxotetrapyrrole was anaTyzed by GC. In addition to the succinimide and maleimide products, an incomplete degradation product appears with a $R_{t} 8.30$ min. The oxidation products from the bile pigments $\underset{\sim \sim \sim}{20 a}, \underset{\sim \sim \sim}{20 b}, \underset{\sim}{21 a}$, and $\underset{\sim}{21} \mathrm{~b}$ were analyzed by TLC. $33,34,37$ In addition to the succinimide and 3-ethyt4-methylmaleimide, 3-(2-methoxycarbonylethy 1)-4-methy1-1H-pyrrole2,5-dione (hematinic acid methyl ester) was seen at $R_{f} 0,50$. 


\section{Acknowledgement.}

This work was supported in part by the Division of Biological Energy Conversion and Conservation (DOE) and the National Institute of General Medical Sciences, DHHS, GM 28994. The $360 \mathrm{MHz}$ MMR studies were carried out at the University of California, Davis, NMR Facility, under the auspices of National Science Foundation Grant CHE 79-04832. 


\section{References}

(1) Gossauer, A. Tetrahedron $1983,39,1933$.

(2) Fujita, Y.: Hattori, A. J. Biochem. (Tokyo) 1962, 51, 89.

(3) Fujita, Y.; Hattori, A. J. Gen. Appl. Microbiol. (Tokyo) 1963, 9, 253.

(4) Crespi, H. L.; Boucher, L. J.; Norman, G. D.; Katz, J. J.; Dougherty, R. C. J. Am. Chem. Soc. 1967, 89, 3642.

(5) Cole, W. J.; Chapman, D. J.; Siegelman, H. W. J. Am. Chem. Soc. 1967, 89, 3643 .

(6) O' Eocha, C. Biochemistry 1963, 2, 375.

(7) Cole, W. J.; Chapman, D. J.; Siegelman, H. W. Biochemistry 1968, ㄱ, 2929.

(8) Rudiger, W.; O Carra, P.; 0 hE sha, C. Nature (London) 1967, 215, 1477.

(9) Gossauer, A.; Hirsch, W. Justus Liebigs Ann. Chem. 1974, 1496.

(10) Gossauer, A.; Hinze, R.-P. J. Org. Chem. 1978, 43, 283.

(11) Gossauer, A.; Hinze, R.-P.; Kutschan, R. Chem. Ber. 1981, 114, 132.

(12) Lagarias, J. C.; Glazer, A. I.; Rapoport, H. J. Am. Chem, Soc. 1979, 101,5030 .

(13) Lagarias, J. C.; Rapoport, H. J. Am. Chem. SoC. 1980, 102, 4821.

(14) Schoenleber, R. W.; Leung, S.-L.; Lunde11, D. J.; Glazer, A. N.;

Rapoport, H. J. Am. Chem. Soc. 1983, 105, 4072.

(15) Brockman, H., Jr.; Knobloch, G. Chem. Ber. 1973, 106, 803.

(16) Glazer, A. N.; Fang, S. J. Biol. Chem. 1973, 248, 659.

(17) Klein, G.; Rudiger, W. Justus Liebigs Ann. Cliem. 1978, 267.

(18) Karplus, M. J. Am. Chem. Soc. 1963, 85, 2870.

(19) Schoch, S.; Klein, G.; Linsenmeier, U.: Rudiger, W. Justus Liebigs 
Ann. Chem. 1976, 549.

(20) van Leusen, A. M.; Siderius, H.; Hoogenboom, B. E.; van Leusen, D. Tetrahedron Lett. 1972, 5337.

(21) Chamberlin, K. S.; LeGoff, E. Heterocycles 1979, 12, 1567.

(22) Bonnett, R.; Fuckley, D. G.; Hamzetash, D. J. Chem. Soc., Perkin Trans. I 1981, 322.

(23) Plieninger, H.; Decker, M. Justus Liebigs Ann. Chem. 1956. 598, 198.

(24) Gossauer, A.; Blacha-Puller, M. Justus Liebigs Ann. Chem. :381, 1492.

(25) Plieninger, H.; Preuss, I. Justus Liebigs Ann. Chem. 1983, 585.

(26) PI ieninger, H.; Preuss, I. Tetrahedron Lett. 1982, 23, 43.

(27) PTieninger, H.; Kurze, J. Justus Liebigs Ann. Chem. 1964, 680, 60.

(28)' Gossauer, A.; Weller, J.-P. Chem. Ber. 1978, 111, 486.

(29) Jackson, A. H.; Kenner, G. W.; Smith, K. M. J. Chem. Soc. C 1971, 502.

(30) Gossauer, A.; Miehe, D. Justus Liebigs Ann. Chem. 1974, 352.

(31) Golden, J. H.; Linstead, R. P. J. Chem. Soc. 1958, 1732.

(32) Rudiger, W. Hoppe-Seyler's Z. Physiol. Chem. 1969, ?j0, 1291.

(33) Reindel, F.; Hoppe, W. Chem. Ber. 1954, 87, 1103.

(34) Ficken, G. E.; Johns, R. B ; Linstead, R. P. J. Chem. Soc. 1956, 2672.

(35) Gray, C. H.; Lemmon, G. A.; Nicholson, D. C. J. Chem. Soc. C 1967, 178.

(36) Gossauer, A.; Weller, J.-P. J. Am. Chem. Soc. 1975, 100, 5928.

(37) Killilea, S. D.; O Carra, P. J. Chromatogr. 1978, 156, 338.

(38) Woodward, R. B.; Sondheimer, F.; Taub, D.; Heusler, K.;

McLamore, W. M. J. Am. Chem. Soc. 1952, 74, 4223.

(39) Anderson, A. G.; Stang, P. J. J. Org. Chem. 1976, 41, 3034.

(40) Gerrard, W.; Nechvatal, A.; Wyvi11, P. L. Chem. and Ind. 1947, 437.

(41) Brown, C. A. Synthes is 1975, 326.

(42) Robinson, R. 1. Chem. Soc. 1916, 109, 1038. 
(43) Fieser, L. F.; Fieser, M. "Reagents for Organic Synthesis"; Vol. I; John Wiley and Sons: New Yor., 1967; p. 197.

(44) Plieninger, H.; Lerch, U. Justus Liebigs Ann. Chem. 1966, 698, 196.

(45) Siede1, w. Hoppe-Seyler's Z. Physiol. Chem. 1935, 237, 8.

(46) Plieninger, H.; Ehl, K.; K1inga, K. Justus Liebigs Ann. Chem. 1971, 743, 112 .

(47) Fischer, H.; Orth, H. "Die Chemie des Pyrrols"; Vol. I;

Akademische Verlagsgese11schaft M.B.H.: Leipzig, 1934; p. 398.

(48) Quistad, G. B.; Lightner, D. A. J. Chem. Soc., Chem. Commun. 1971, 1099.

(49) Whit7ock, H. W.; Hanauer, R. J. Org. Chem. 1968, 33, 2169.

(50) Petryka, Z. J.; Watson, C. J. Tetrahedron Lett. 1967, 5323.

(51) Muir, H. M.; Neuberger, A. Biochem. J. 1949, 45, 163.

(52) Fischer, H.; Orth, H. "Die Chemie des Pyrrols"; Vol. I;

Akademische Verlagsgese11schaft M.B.H.: Leipzig, 1934; p. 49. 
Part Two

Bilin Attachment Sites in the $\alpha$ and $\beta$ Subunits of e-Phycoerythrin:

Structural Studies on the Singly-Linked Phycoerythrobilins

Abstract

Five phycoerythrobilin peptides were prepared from Porphyridium cruentum B-phycoerythrin by a combination of tryptic and themoiysin digestion and isolated under mild conditions without alteration in the spectroscopic properties of the bilin. High resolution $l_{H}$ MMR spectral analys is of four of the chromopeptides has provided direct proof that all of the singly-linked phycoerythrobilins contain a thioether bond to the 3'-position of ring $A$. The present study confirms the major structural features of the phycoerythrobilin chromophore and indicates the probable stereochemical assignments for all of the chiral centers for every singly-linked phycoerythrobilin on the $\alpha$ and $B$ subunits of B-phycoerythrin. 
The intenseiy red phycoerythrins serve as major light harvesting proteins in numerous cyanobacteria as well as in two algal groups, the Rhodophyta (red algae) and the cryptomonads. 'Rhodophytan phycoerythrins carry two types of covalently bound prosthetic groups, phycoerythrobilins and phycourobilins. ${ }^{2}$ B-Phycoerythrin from the unicellular red alga Porphyridium cruentum, the source of the bilin peptides we have investigated, has the polypeptide composition $(\alpha \beta)_{G} \gamma$, with $\alpha$ and $\beta$ subunits of 17,000 daltons and $\gamma$ subunit of 30,000 daltons. ${ }^{3-5}$ The a subunit contains two phycoerythrobilins, the $B$ subunit contains three phycoerythrobilins, and the $\gamma$ subunit contains two phycoerythrobilins and two phycourobilins as determined by spectroscopic studics of the isolated subunits. ${ }^{4}$ It is important to appreciate, then, that isolation and characterization of 9 distinct peptides is required to fully establish the structure of all of the polypeptide linked bilins.

All of the phycoerythrobilins on the $\alpha$ and $B$ subunits are accounted for in five chromopeptides derived from trypsin digestion of B-phycoerythrin. ${ }^{6}$ One of these chromopeptides, B-3T is the subject of part three of this thesis. Herein, we report a detailed structural study of the four remaining phycoerythropeptides obtained upon trypsin cleavage of the $\alpha$ and $\beta$ subunits of B-phycoerythrin.

B-Phycoerythrin was isolated and purified from Porphyridium cruentum by a combination of ammonium sulfate precipitation, DEAEcellulose chromatography, and gel filtration. ${ }^{4}$ Trypsin digestion was carried out on the intact $(\alpha \beta)_{6} \gamma$ complex of purified B-phycoerythrin. ${ }^{6}$ From work on the isolated subunits, the origin of the chromopeptides could be ascertained from the Sephadex elutior profiles. "Thus, the $\alpha-1, \alpha-2$, and $E-1$ chromepeptides were isolated. The $B-2$ chromopeptide 
from trypsin cleavage was further digested with thermolys in and fractionated on Sephadex to give the $\beta-2 T$ chromopeptide investigated here.

Amino acid analyses and sequential Edman degradations served to establish the amino acid sequences of the chromopeptides as follows:

$$
\begin{aligned}
& \alpha-1: \quad \text { Cys-Tyr-A.rg } \\
& \text { PEB } \\
& \alpha-2: \quad \text { Leu-Cys-Val-Pro-Arg } \\
& \text { B-1; Met-A1a-A1a-Cys-Leu-Arg } \\
& \text { PEB } \\
& \text { B-2T: Phe-Ala-Ala-Gly-Asp-Cys-Thr-Ser } \\
& \text { PEB }
\end{aligned}
$$

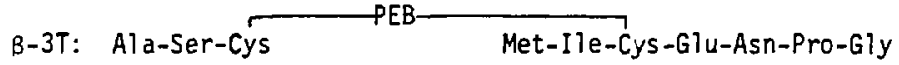

where the designations $\alpha$ and $\beta$ refer to the subunit from which the peptide was derived, the number refers to the relative $\mathrm{N}$-terminal position of the phycoerythrobilin (PEB) p:ptide fragment found, and the $T$ indicates a thermolysin cleavage in addition to trypsin cleavage. In addition to the sequence data, the Edman degradations also indicated the attachment site on the peptide of the phycoerythrobilin. The loss of the red color due to the tetrapyrrole was always concomitant with the loss of the indicated cysteine residues suggesting a thioether linkage between the 
peptide and the chromophore. The absence of any change in the UV/vis spectra shows that no alteration in the chroniophore structure has, occurred during the enzymatic cleavages.

The chromophore structure and peptide linkage of the $\beta-3 T$ chromopeptide is discussed in part three of this thesis. The remaining four peptides were further purified by HPLC (Figures 1 and 2). Reverse-phase chromatography was done using an isocratic sodium phosphate bufferacetonitrile mixture on an U1trasphere ODS column. Our studies indicated that a high buffer salt concentration and isocratic conditions were essential for the requisite resolution. The $\alpha-2$ and the $\beta-1$ chromopeptides were incompletely separated by gel filtration, but reverse phase chromatography yielded very pure fractions for NPIR analysis. The B-2T chromopeptide contained a major impurity (20\%) of unknown origin which was removed upon HPLC purification. The chromatography elution profiles at $200 \mathrm{~nm}$ showed that the isolated chromopeptides were the major peptide containing fractions. Each HPLC fraction was desalted by adsorption onto a LiChrosorb $\mathrm{C}-18$ column equilibrated with $10 \mathrm{mM}$ aqueous TFA and eluted with a mobile phase of $50 \%$ acetonitrile and $50 \%$ aqueous $10 \mathrm{mM}$ TFA. The use of this volatile acid allowed us control the acid concentration of the final MMR sample without the introduction of interfering proton resonances. During the desalting procedure, some chromophore cieavage $(0.5 \%)$ was seen, al though both free chromophore and free peptide were well separated from the chronlopeptide fractions. The UV/vis spectra conclude the integrity of the chromophore at this final purification stage as well as provide evidence for the identity of the tetrapyrrole in all four chromopeptides (Figures 3 and 4).

Amino acid sequencing data on the purified ce-1 chronopeptide 


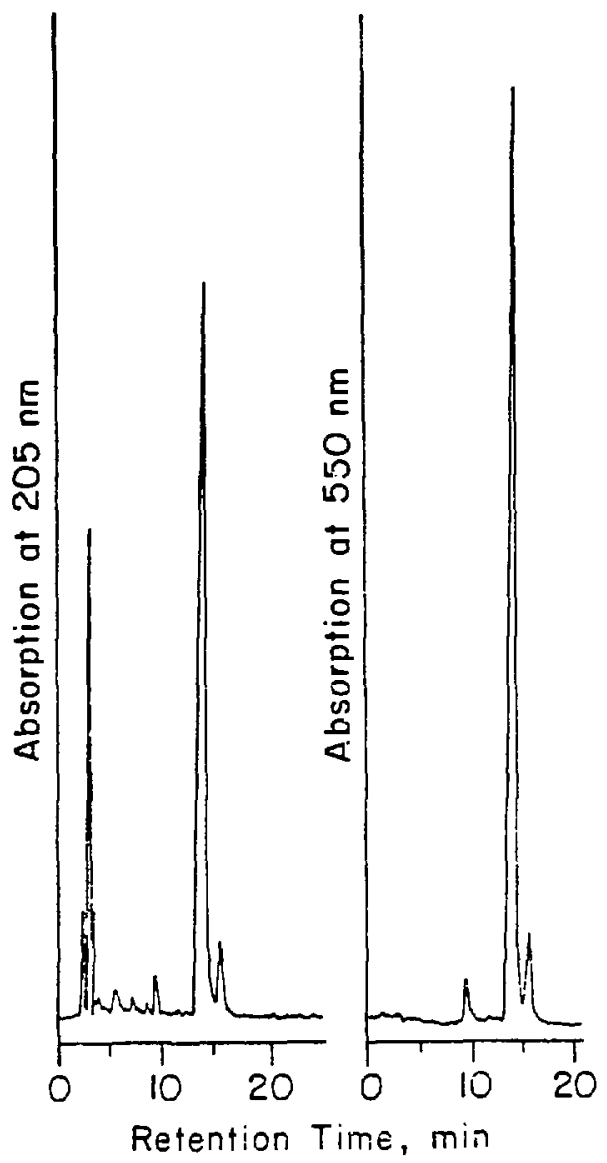

Figure 1. High pressure liquid chromatography of the $a-1$ phycoerythrobilitripeptide $\underline{B}$ on tn Uitrasphere ODS columin $(4.6 \times 250 \mathrm{~mm}, 5 \mu \mathrm{m})$ using $22.5 \% \mathrm{CH}_{3} \mathrm{CN}$ and $77.5 \% 0.10 \mathrm{M}$ $\mathrm{Nath}_{2} \mathrm{Fl}_{4}$ buffer, $\mathrm{pH} 2 . \mathrm{i}$ wit a $1.0 \mathrm{~mL} / \mathrm{min}$ fles rate. 


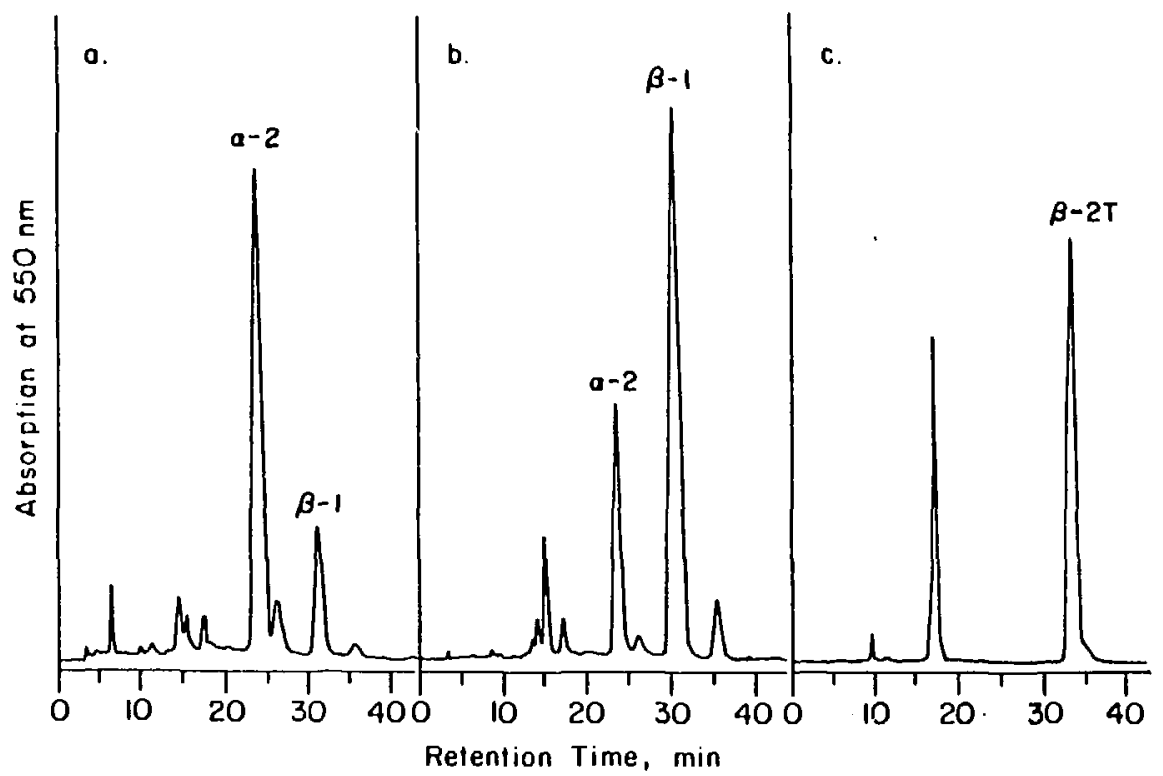

Figure 2. High pressure liquid chromatography of phycoerythrobilipeptides (a) $\alpha-2$, (b) $\beta-1$, (c) $\beta-2 T$ on an UTtrasphere ODS columin $(4.6 \times 250 \mathrm{~mm}, 5 \mu \mathrm{m})$ using $22.5 \% \mathrm{CH}_{3} \mathrm{CN}$ and $77.5 \%$ $0.10 \mathrm{M} \mathrm{NaH}{ }_{2} \mathrm{PO}_{4}$ buffer, $\mathrm{pH} 2.1$ with a $1.0 \mathrm{~mL} / \mathrm{min}$ flow rate and detection at $550 \mathrm{~nm}$. 


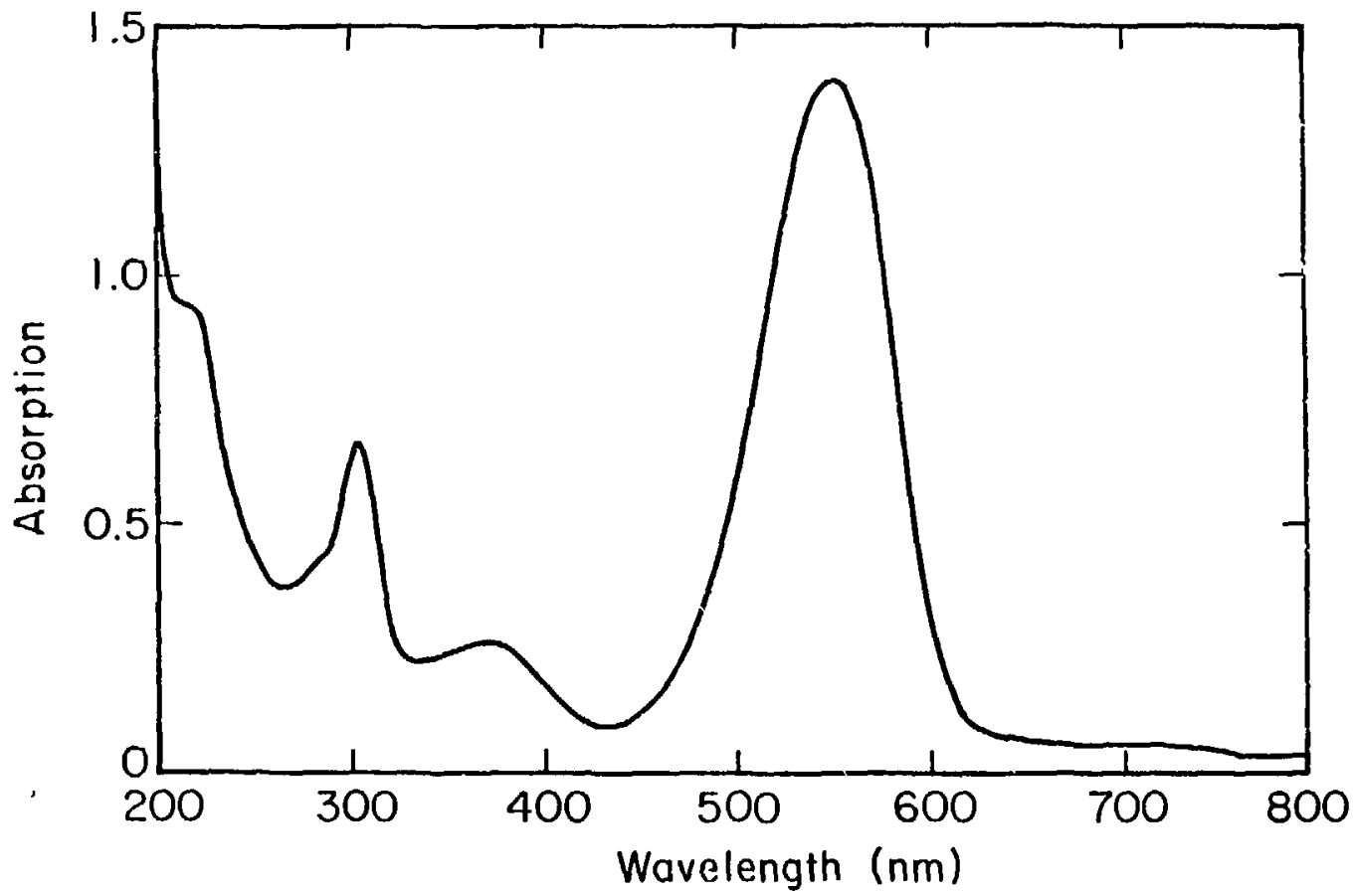

Figure 3. The absorption spectrum in $10 \mathrm{mM}$ aqueous TFA of the HPLC purified $\alpha-1$ phycoerythrobilitripeptide $\underset{\sim}{8}(c=30.0 \mu \mathrm{M})$.

ఉ 
44

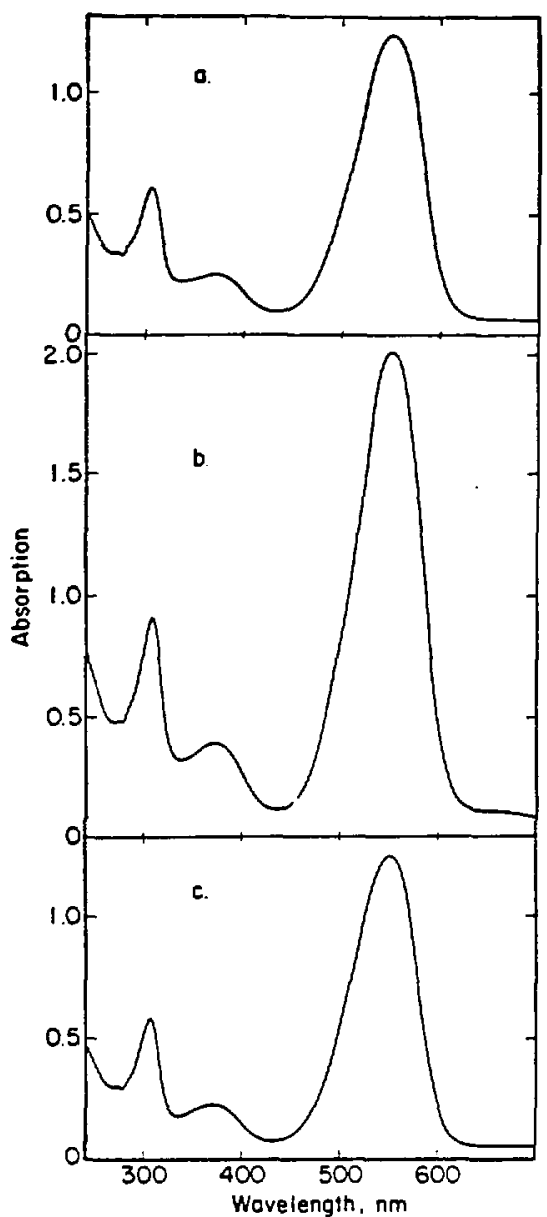

Figure 4. The absorption spectra in $10 \mathrm{mM}$ aqueous TFA of the HPLC purified phycoerythrobilipeptides (a) $\alpha-2, c=26.2$ HM; (b) $\beta-1, c=42.2 \mu M$; (c) $\beta-2 T, c=26.4 \mu M$. 
established that the sequence was Cys-Tyr-irg. In order to facilitate NMR analysis and to see what effects might be caused by chromophorepeptide interactions, the tripeptide was synthesized (Chart 1). The synthesis was achieved using a mixed anhydride method on fully protected amino acid residues to satisfy the purity requirements of the high resolution NHR study. 7

The amino acid derivatives were synthesized according to known methods. $8-12$ The couplings were done in such a manner as to avoid racemization which would result in diastereomeric mixtures complicating the NMR anaTysis, and the protected di- and tri-peptides were purified by HPLC. Final deprotection with anhydrous hydrogen fluoride removed all blocking groups except the acetamidomethyl, which was chosen to avoid any complicating thiol oxidation. ${ }^{8,13}$ Although the yields were generaliy low, the HPLC analyses showed high purity indicating that the losses were probably due to the extensive handling necessary in the rigorous purification procedures.

The NMR spectra were taken in $10 \mathrm{mM}$ TFA in $\mathrm{O}_{2} \mathrm{O}$. Although we considered that aggregation might be a problem, the circular dichroism spectra showed no concentration effects in aqueous buffer up to the concentration at which the NMR spectra were recorded. ${ }^{14}$ Previous results with phycocyanobilin-bearing peptides suggested that pyridine might be a better NMR solvent, ${ }^{14,15}$ but isomerization of the phycoerythrin to a urobilin-type structure prevented meaningful NMR analysis in pyridine.

The 360 MHz MMR spectra of the chromopeptide $\alpha-1 \underset{\sim}{(8)}$ and the corresponding synthetic tripeptide 7 are shown in Figure 5 . In contrast to what occurs with phycocyanobilipeptides in $D_{2} 0$, very littie broadening 
Chart 1. Repetitive Mixed Anhydride Synthesis of Tripeptide?.

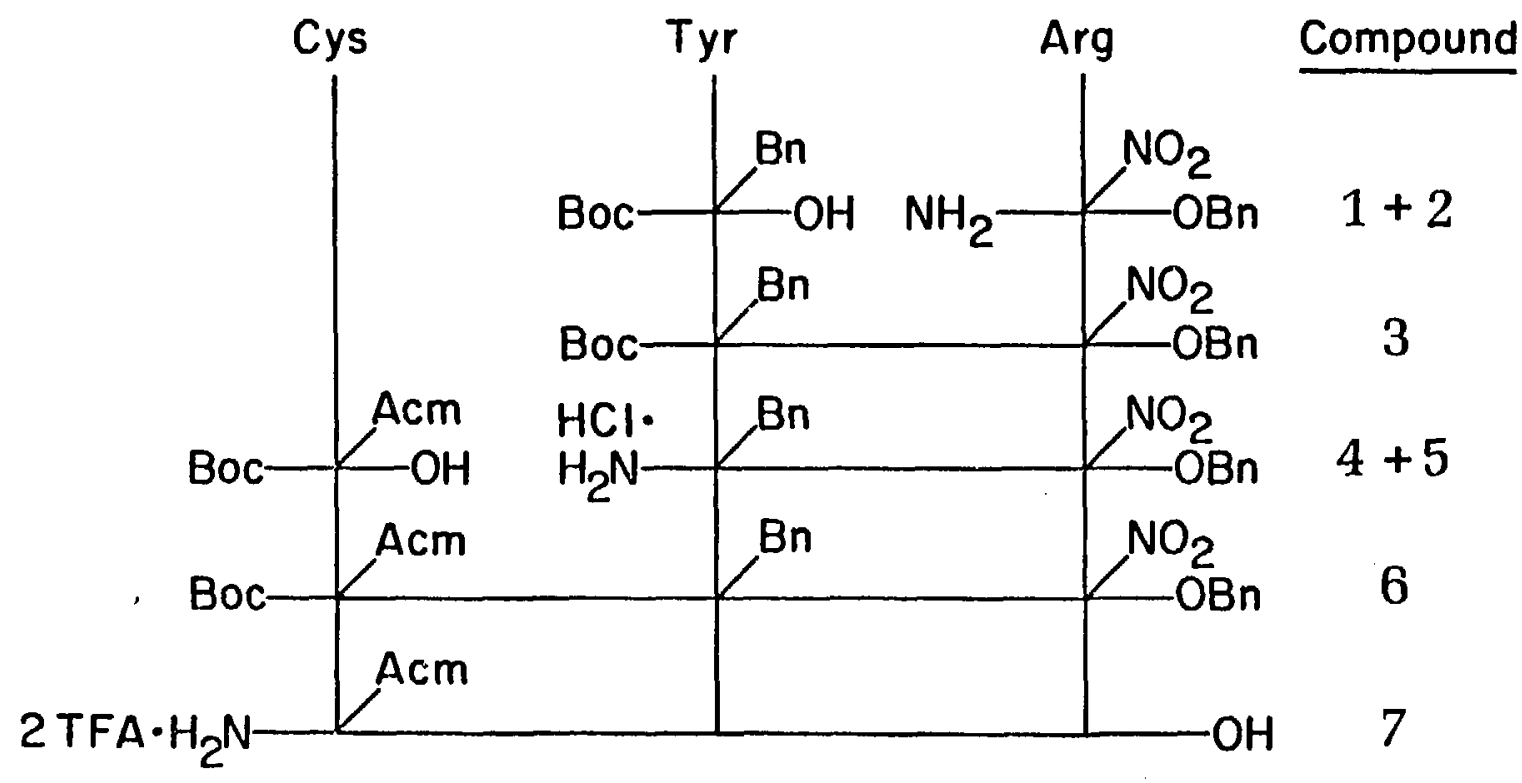




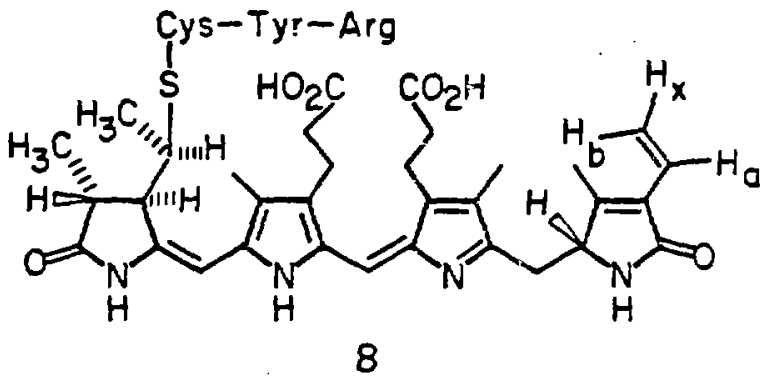

$\alpha-1$ Phycoerythrobilitripeptide 


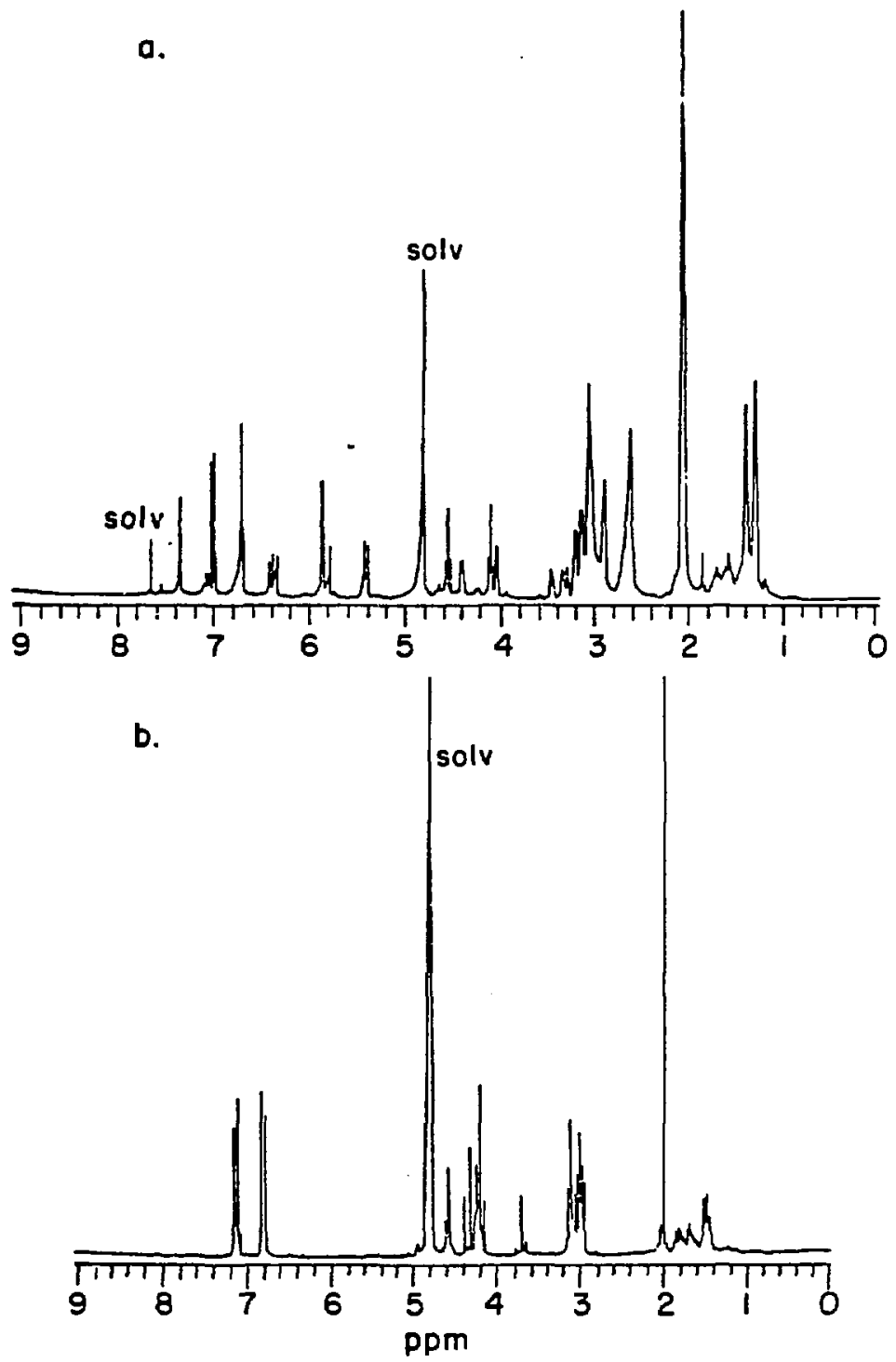

Figure 5. (a) The $360 \mathrm{MHz}{ }^{l_{H}}$ MMR spectrum of the $\alpha$-1 phycoerythrobilitripeptide 8 ( $c=4.4 \mathrm{mM}$ ) in $10 \mathrm{mM} \mathrm{TFA}$ in $\mathrm{D}_{2} \mathrm{O}$ at $25^{\circ} \mathrm{C}$; (b) The $250 \mathrm{MHz} \mathrm{C}^{\mathrm{H}}$ MHR spectrum of synthetic tripeptide $?(\mathrm{c}=5.0 \mathrm{mM})$ in $10 \mathrm{~mm}$ TFA in $\mathrm{D}_{2} \mathrm{O}$ at $25^{\circ} \mathrm{C}$. 
of the resonances due to the chromophore is seen. ${ }^{14}$ The corresponding chemical shifts of the amino acid residues for chromopeptide $\alpha-1$ and the synthetic tripeptide all fall within $0.2 \mathrm{ppm}$ (Table I). Considering the possible effects of such a highly conjugated system in close proximity to the peptide, the chemical shift agreement is surprisingly close. However, because of this agreement, little speculation on conformational features can be made. There is a general pattern of shielding of the peptide resonances, indicating that some internal hydrogen bonding or hydrophobic interactions may hold the peptide chain over the tetrapyrrole in the shielding regian of the ring current effects. However, the pattern is not absolute and no data on the conformation of the bilin in such a complex or in such a solvent are available. ${ }^{16}$

The close agreement of the chemical shifts of the tyrosine aromatic resonances precludes the possibility of an ester linkage between the phenoli, oxygen and a propionic acid side chain. Therefore, since a free arginine guanidine group is essential for tryps in cleavage, the thioether linkage is shown to be the only covalent bond to the chromophore in the $\alpha-1$ chromopeptide.

Detailed MMR spectral analysis of the chromophore resonances provides information not previously available (Table II). The singlets at 7.34 and $5.86 \mathrm{ppm}$ represent the methine protons at $\mathrm{C}-1 \mathrm{C}$ and $\mathrm{C}-5$, respectively. No deuterium exchange is seen at these, or any other possibly enolizable protons under the aqueous acid conditicns employed. This is in marked contrast to the exchange seen in the phycocyanobilin chromopeptide ${ }^{14}$ and a previous report on the phycoerythrobilin chromophore. ${ }^{17}$ The lability of certain protons having been documented, we interpret our results as an indication that no isomerization has occurred during the mild conditions 
Table I. $1_{H}$ NMR Assignments for the Peptide Moiety of the $\alpha-1$ Phycoerythrobilipeptide 8 and the Synthetic Peptide 7 in $10 \mathrm{~mL}$ TFA in $\mathrm{D}_{2} \mathrm{D}$ at $25^{\circ} \mathrm{C}$.

\begin{tabular}{|c|c|c|}
\hline$\underset{\sim}{8}$ & 7 & assignment \\
\hline $6.98(d, 8.4)$ & $7.11(d, 8.5)$ & Tyr 2,6 Ar-4 \\
\hline $6.71(d, 8.4)$ & $6.78(d, 8.5)$ & Tyr 3,5 Ar-H \\
\hline $4.55(t, 7.2)$ & $4.54(t, 7.7)$ & Cys $\alpha-C H$ \\
\hline $4.10(t, 6.3)$ & $4.20(t, 4.5)$ & Tyr $\alpha-C H$ \\
\hline $4.03(\mathrm{dd}, 5.1,8.5)$ & $4.23(t, 5.0)$ & $\operatorname{Arg} \alpha-\mathrm{CH}$ \\
\hline $3.2(\mathrm{~m})$ & $3.1(t, 6.9)$ & Arg $\delta-\mathrm{CH}_{2}$ \\
\hline $3.14(m)$ & $3.04(d, 5.6) ; 2.99(d, 7.2)$ & Tyr $\mathrm{B}-\mathrm{CH}_{2}$ \\
\hline $2.90(d, 7.2)$ & $2.98(d, 7.7)$ & Cys $\mathrm{B}-\mathrm{CH}_{2}$ \\
\hline $1.55-1.75(\mathrm{~m})$ & $1.47-1.77(\mathrm{~m})$ & $\operatorname{Arg} B, \gamma-\mathrm{CH}_{2}$ \\
\hline
\end{tabular}


Table II. ${ }^{1} H$ NMR Assignments of the Bilin Moiety of the a-1 Phycoerythrobilitripeptide 8 in $10 \mathrm{mM}$ TFA in $\mathrm{D}_{2} \mathrm{O}$ and the Free Pigment 9 in $\mathrm{CDCl}_{3}$ at $25^{\circ} \mathrm{C}$.

\begin{tabular}{|c|c|c|}
\hline$\underline{8}$ & $\underline{g}^{a}$ & assigninent \\
\hline $7.34(\mathrm{~s})$ & $6.68(\mathrm{~s})$ & $10-H$ \\
\hline $6.37(\mathrm{dd}, 11.7,17.9)$ & $6.40(d d, 12,18)$ & $18-\mathrm{H}_{\mathbf{a}}$ \\
\hline $5.86(s)$ & $5.84(s)$ & $5-H^{\circ}$ \\
\hline $5.82(\mathrm{dd}, 1.4,17.9)$ & $6.17(d d, 2,18)$ & $18-H_{b}$ \\
\hline $5.40(d d, 1.4,11.7)$ & $5.36(d d, 2,12)$ & $18-H_{x}$ \\
\hline $4.40(d d, 4.2,7.9)$ & $4.28(m)$ & $16-\mathrm{H}$ \\
\hline $3.44(\mathrm{~m}, 3.0,7.0)$ & $b$ & $3^{\prime}-\mathrm{H}$ \\
\hline $3.30(d d, 4.2,14.1)$ & $2.8-3.0(\mathrm{~m})$ & $15-\mathrm{H}$ \\
\hline $3.10(m, 3.0,3.5)^{c}$ & $b$ & $3-H$ \\
\hline $3.07(\mathrm{~m})$ & $2.8-3.0(\mathrm{~m})$ & $8,12-\mathrm{CH}_{2} \mathrm{CH}_{2} \mathrm{CO}_{2} \mathrm{H}$ \\
\hline $2.93(m, 7.9,14.1)^{c}$ & $2.8-3.0(\mathrm{~m})$ & $15-\mathrm{H}$ \\
\hline $2.69(m, 3.5,7.4)$ & b & $2-$ is \\
\hline $2.64(\mathrm{~m})$ & $2.4-2.7(\mathrm{~m})$ & $8.12-\mathrm{CH}_{2} \mathrm{CH}_{2} \mathrm{CO}_{2} \mathrm{H}$ \\
\hline $2.08(\mathrm{~s})$ & $2.03(\mathrm{~s})$ & \\
\hline $2.06(5)$ & $2.03(\mathrm{~s})$ & $7,13,17-\mathrm{CH}_{3}$ \\
\hline $2.055(\mathrm{~s})$ & $1.98(\mathrm{~s}))$ & \\
\hline $1.39(d, 7.0)$ & b & $3^{\prime}-\mathrm{CH}_{3}$ \\
\hline $1.28(d, 7.4)$ & $1.42(d, 7.0)^{b}$ & $2-\mathrm{CH}_{3}$ \\
\hline
\end{tabular}

arom reference 20 .

${ }^{b}$ This chemical shift is not strictly analogous or no longer exists due to the presence of the ethylidene.

${ }^{c}$ Overlapping resonances are seen, but coupling constants are abstracted from the coupled protons. 
used in this study.

The ABX pattern at $6.37,5.82$, and $5.40 \mathrm{ppm}$ with coupling constants of $1.4,11.7$, and $17.9 \mathrm{~Hz}$, respectively, is direct proof of the presence of a side chain vinyl group in the native chromophore. The mild procedure used for isolation of the chromopeptide precludes the possible generation of this vinyl group, as evidenced by the intact nature of the thioether linkage. The chemical shifts and coupling constants correspond well with those found for the free chromophore 9 despite the solvent differences. $18,19,20$

From a comparison with the synthetic peptide, the $\alpha$-protons of the amino acid residues can be assigned. Further comparisons and decoupling experiments then allow the complete assignment of the amino acid resonances (Table 1). By difference, we can assign the doublet of doublets at 4.40 $\mathrm{ppm}(\mathrm{J}=4.2,7.9 \mathrm{~Hz})$ to the proton at $\mathrm{C}-16$, indicating the saturated nature of the adjacent methyiene position. Double irradiation experiments show that the protons at $\mathrm{C}-15$ lie at 3.30 and approximately $2.9 \mathrm{ppm}$. The higher field $\mathrm{C}-15$ proton is a doublet of doublets with coupling constants of $4.2 \mathrm{~Hz}\left(\mathrm{~J}_{\text {vicinal }}\right)$ and $14.1 \mathrm{~Hz}$ (J $\left.\mathrm{J}_{\text {geminal }}\right)$. The other $\mathrm{C}-15$ proton is obscurred by overlapping resonances, al though the decoupling data allow the approximate chemical shift assignment, as irradiation at $2.9 \mathrm{ppm}$ collapses both the $\mathrm{C}-16$ and the other $\mathrm{C}-15$ proton. Its coupling constants must be $7.9 \mathrm{~Hz}\left(\mathrm{~J}_{v i c i n a l}\right)$ and $14.1 \mathrm{~Hz}\left(\mathrm{~J}_{\text {geminal }}\right)$. The 1 ine widths of the $\mathrm{C}-15$ and $\mathrm{C}-16$ protons are considerably larger than those seen for the other protons, which indicates restricted rotation about this bond, consistent with a rigid preferred conformation due to interna $i$ hydrogen bonding despite the aqueous acid solvent. Perhaps a more plausible explanation, however, would be that lypcphilic interactions 


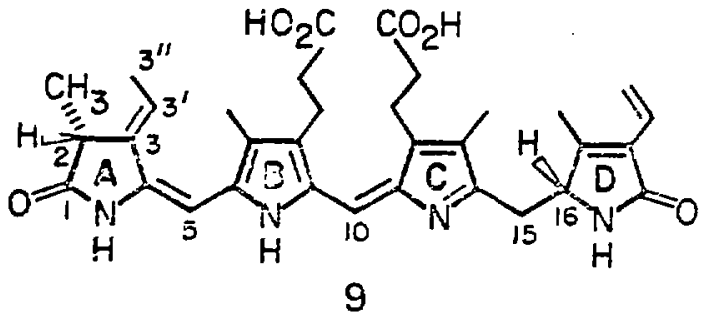

Phycoerythrobilin 
between the two end rings of the tetrapyrrole itself are the cause of the rotational constraints.

With the exception of those of ring $A$, the remaining tetrapyrrole substituents are assigned by comparison with phycoerythrobilin NMR data previously published. $18,19,20$ The propionic arid methylenes appear as unresolved multiplets centered at $3.07 \mathrm{ppm}$ for the $\beta$ - and $2.64 \mathrm{ppm}$ for the $\alpha$-methylene groups. The three methy 1 groups at $\mathrm{C}-7, \mathrm{C}-13$, and $\mathrm{C}-17$ are not completely resolved in cur spectra. A resolution enchancement technique (sinusoidal mutiplication of the free induction decay curve) shows the presence of the three at $2.055,2.06$, and $2.08 \mathrm{ppm}$.

The substitution pattern about ring $A$ was ascertained by decoupling experiments. Previous results ${ }^{2}$ suggest that a methyl group is at $C-2$ and an ethyl group is at $\mathrm{C}-3$, with a thioether linkage at $\mathrm{C}-3^{\prime}$. We provide the first direct proof of this assignment. The $\mathrm{C}-2$ methyl group appears as a doublet at $1.28 \mathrm{ppm}$ as determined by corre?ation with the phycocyanin work. ${ }^{14}$ Irradiation at this position causes the $\mathrm{C}-2$ proton at $2.69 \mathrm{ppm}$ (Figure 6) to collapse to a doublet. The observed coupling constant between the $c-2$ and $c-3$ protons is $3.5 \mathrm{~Hz}$. The $c-3$ proton is obscurred by the propionic acid Bumethylene protons. However, irradiation at 3.1 pprin causes a smal1 change in the appearance of the $c-2$ proton, indicating the approximate chemical shift of the $\mathrm{C}-3$ proton. The overlapping resonances do not aliow us to see a change in the $c-\hat{3}$ proton when the $C-2$ proton is irradiated; however, the $\mathrm{C}-2$ methyl group does collapse to a singlet when this irradiation is done (Figure 6 ). The $[-2$ methyl doublet allows the direct reading of the coupling constant between the $\mathrm{C}-2$ methyl and proton as $7.4 \mathrm{~Hz}$.

A similar pattern is seen for the $\left[-3^{\prime}\right.$ proton at 3.44 ppin. This 


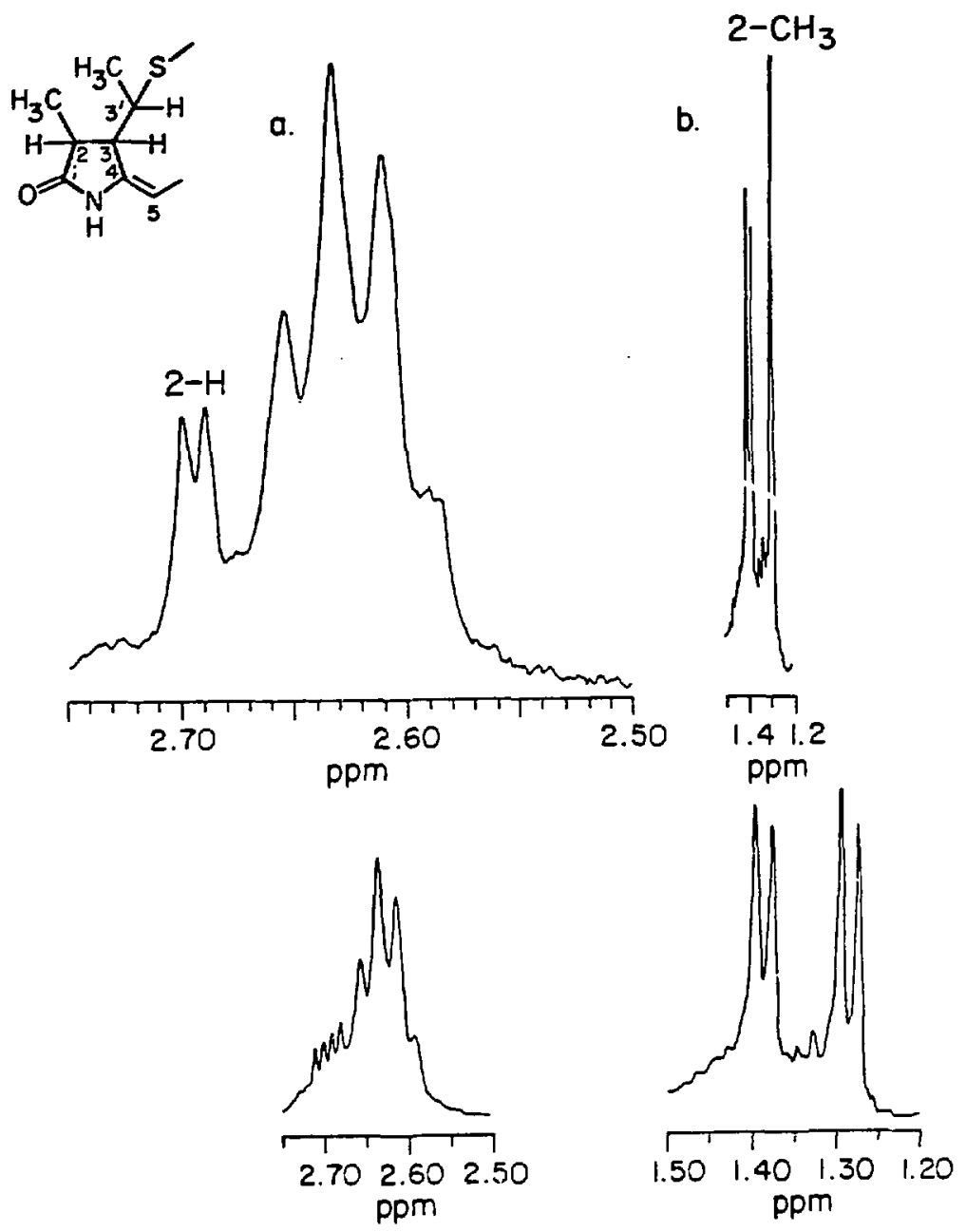

Figure 6. The partial $360 \mathrm{MHz}{ }^{3} H$ NMR spectrum of the $\alpha-1$ phyco erythrobilitripeptide $\underset{\sim}{8}$ in $10 \mathrm{mM}$ TFA in $\mathrm{D}_{2} \mathrm{O}$ at $25^{\circ} \mathrm{C}$ (a) after irradiation of the dowblet at $1.28 \mathrm{ppm}$; (b) after irradiation of the multiplet at $2.69 \mathrm{ppm}$. 
proton initially appears as a multiplet with an apparent four line pattern (Figure 7). Irradiation at the $\mathrm{C}-3$ proton only causes slight changes in the appearance of the $\mathrm{C}-3^{\prime}$ proton. The collapse should be to a quartet, but the imprecise nature of the $\mathrm{C}-3$ proton irradiation, because of overlapping resonances, did not allow us to make this direct correlation. Irradiation of the $\mathrm{C}^{-3^{\prime}}$ methyl group at $1.39 \mathrm{ppm}$ collapses the $\mathrm{C}-3^{\prime}$ proton to a doublet with $\mathrm{J}_{3,3^{\prime}}=3.0 \mathrm{~Hz}$. Irradiation of the $\mathrm{C}-3^{\prime}$ proton shows no change in the $\mathrm{C}-3$ region but does collapse the $\mathrm{C}-3^{\prime}$ methyl group signal to a singlet. Direct reading of the coupling constant of the initial doublet gives a value of $7.0 \mathrm{~Hz}$. Therefore, we have provided the first direct proof of the presence and position of the thioether linkage in a phycoerythrobilipeptide.

The $500 \mathrm{MHz}{ }^{1} \mathrm{H}$ NMR spectra of chromopeptides $\alpha-2, \beta-1$, and $\beta-2 T$ are shown in Figure 8. Again, the aqueous acid solvent provides excellent stability for the chromophore and avoids any problems associated with aggregation or with interconverting prototropic isomers of the tetrapyrrole. The chemical shift and coupling constant assignnerits for the bilin portion of all four chromopeptides are shown in Table III. The correspondence between the $\alpha-1, \alpha-2$, and $\beta-1$ chromopeptides is excellent. The largest chemical shift discrepancy is a $0.12 \mathrm{ppm}$ difference in the $3-\mathrm{H}$, while all other chemical shifts agree within $0.04 \mathrm{ppm}$. Likewise, all coupling constants agree Hell, with the largest difference (1.1 Hz) seen in the $J_{15,16}$. Clearly, no gross structural differences are seen. The presence of the $C-18$ vinyl group and the saturation at the $C-15 / C-16$ position of the $\alpha-2$ and $\beta-1$ chromopeptides can be inferred by analogy to the detailed $\alpha-1$ study. Decoupling studies conrirm these assignments as well as allow assignments for the $2-\mathrm{CH}_{3}, 3^{\prime}-\mathrm{CH}_{3}, 2-\mathrm{H}, 3-\mathrm{H}$, and $3^{\prime}-\mathrm{H}$. The exact 


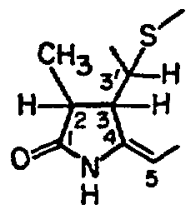

b. $3^{\prime}-\mathrm{CH}_{3}$
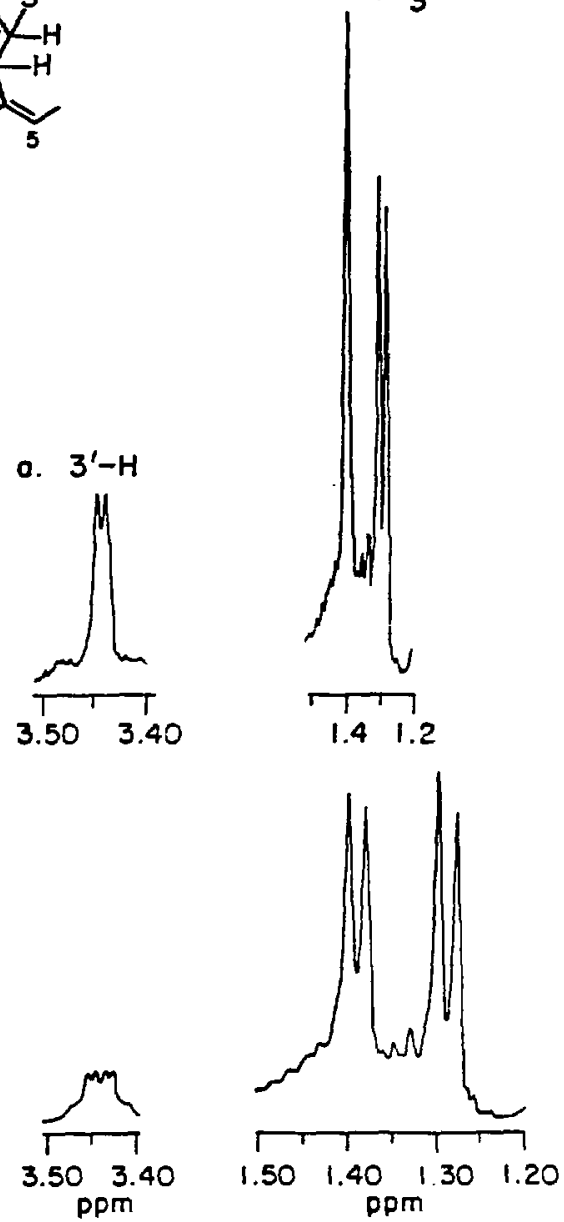

Figure 7. The partial $360 \mathrm{MHz}{ }^{1} \mathrm{H}$ NMR spectrum of the $\alpha-1$ phycoerythrabilitripeptide $\underset{\sim}{8}$ in $10 \mathrm{mM}$ TFA in $\mathrm{D}_{2} \mathrm{O}$ at $25^{\circ} \mathrm{C}$ (a) after irradiation of the doublet at $1.39 \mathrm{ppm}$;

(b) after irradiation of the multiplet at $3.44 \mathrm{ppm}$. 


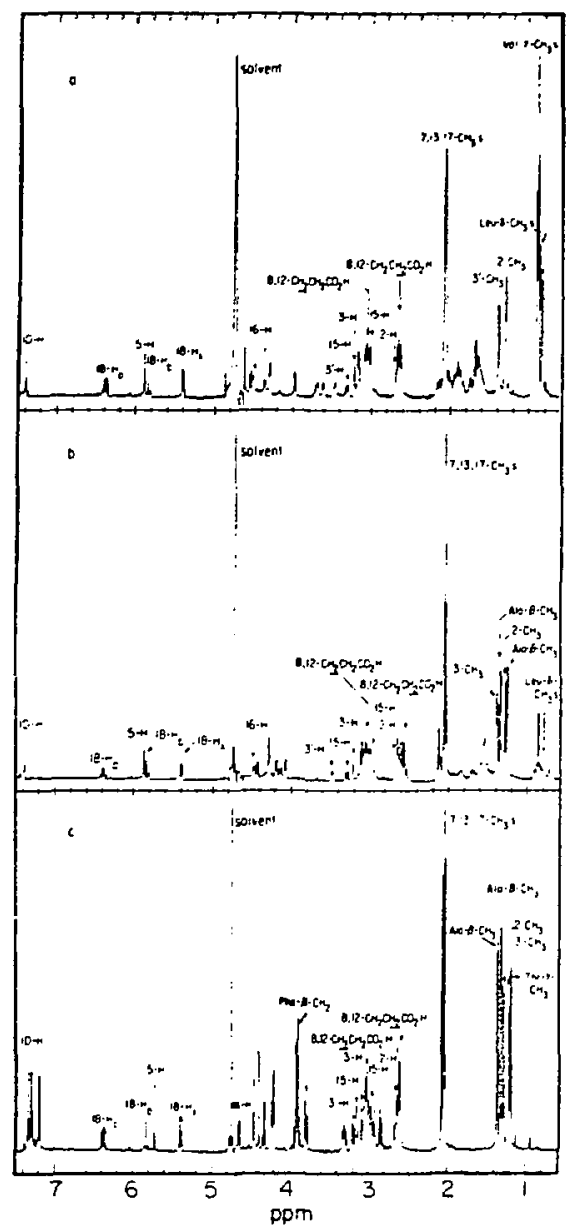

Figure 8. The $500 \mathrm{MHz}{ }^{l_{H}}$ NMR spectra of the phycoerythrobilipeptides (a) $c-2, c=5.2 \mathrm{mM}$; (b) $B-1, c=4.2 \mathrm{mM}$;

(c) $\mathrm{B}-2 \mathrm{~T}, c=2.6 \mathrm{mH}$ in $10 \mathrm{mH}$ TFA in $\mathrm{D}_{2} \mathrm{O}$ at $25^{\circ} \mathrm{C}$. 


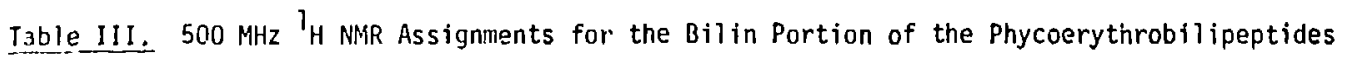
$\alpha-1(4.4 \mathrm{mM}), \alpha-2(5.2 \mathrm{mM}), \beta-1(4.2 \mathrm{mM})$, and $\beta-2 T(2.6 \mathrm{mM})$ in $10 \mathrm{mM}$ TFA in $\mathrm{D}_{2} 0$ at $25^{\circ} \mathrm{C}$.

\begin{tabular}{|c|c|c|c|c|}
\hline$a-1^{a}$ & $a-2$ & $B-1$ & $\beta-2 T$ & Assignment \\
\hline $7.34(\mathrm{~s})$ & $7.39(\mathrm{~s})$ & $7.39(s)$ & $7.34(s)$ & $10-\mathrm{H}$ \\
\hline $6.37(\mathrm{dd}, 11.7,17.9)$ & $6.38(\mathrm{dd}, 11.6, \mathrm{i} 7.8)$ & $6.39(\mathrm{dd}, 11.6,18.0)$ & $6.37(\mathrm{dd}, 11.7,18.0)$ & $18-H_{a}$ \\
\hline $5.86(\mathrm{~s})$ & $5.89(\mathrm{~s})$ & $5.89(\mathrm{~s})$ & $5.73(\mathrm{~s})$ & $5-H^{2}$ \\
\hline $5.82(\mathrm{dd}, 1.4,17.9)$ & $5.83(\mathrm{dd}, 1.5,17.8)$ & $5.83(\mathrm{dd}, 1.5,18.0)$ & $5.82(\mathrm{dd}, 1.7,18.0)$ & $18-H_{b}$ \\
\hline $5.40(d d, 1.4,11.7)$ & $5.39(d d, 1.5,11.6)$ & $5.40(d d, 1.5,11.6)$ & $5.39 ! \mathrm{dd}, 1.7,11.7)$ & $18-H_{x}$ \\
\hline $4.40(\mathrm{dd}, 4.2,7.9)$ & $4.50(\mathrm{dd}, 3.6,8.5)$ & $4.49(\mathrm{dd}, 4.7,8.0)$ & $4.65(\mathrm{dd}, 6.6,7.4)$ & $16-H$ \\
\hline $3.44(\pi, 3.0,7.0)$ & $3.45(\mathrm{~m}, 3.2,7.0)$ & $3.48(m, 3.0,6.9)$ & $3.32(m, 4.0,7.0)$ & $3^{\prime}-\mathrm{H}$ \\
\hline $3.30(\mathrm{dd}, 4.2,14.1)$ & $3.30(d d, 3.6,13.6)$ & $3.30(\mathrm{dd}, 4.7,14.5)$ & $3.13(d d, 6.6,13.9)$ & $15-H$ \\
\hline $3.10(\mathrm{~m}, 3.0,3.5)$ & $3.22(m, 3.2,3.8)$ & $3.22(m, 3.0,3.6)$ & $3.04(m, 3.4,4.0)$ & $3-\mathrm{H}$ \\
\hline $3.07(\mathrm{~m})$ & $3.05(\mathrm{~m})$ & $3.04(\mathrm{~m})$ & $3.03(\mathrm{~m})$ & $8,12-\mathrm{CH}_{2} \mathrm{CH}_{2} \mathrm{CO}_{2} \mathrm{H}$ \\
\hline $2.93(\mathrm{~m}, 7.9,14.1)$ & $3.00(m, 8.5,13.6)$ & $3.02(m, 8.0,14.5)$ & $2.97(m, 7.4,13.9)$ & $15-\mathrm{H}$ \\
\hline $2.69(m, 3.5,7.4)$ & $2.68(\mathrm{~m}, 3.8,7.4)$ & $2.67(m, 3.6,7.4)$ & $2.67(\mathrm{~m}, 3.4,7.4)$ & $2-H$ \\
\hline $2.04(\mathrm{~m})$ & $2.63(2 \times t, 7.2,7.6)$ & $2.63(2 x t, 7.3,7.6)$ & $2.62(2 \times t, 7.2,7.4)$ & $8,12-\mathrm{CH}_{2} \mathrm{CH}_{2} \mathrm{CO}_{2} \mathrm{H}$ \\
\hline $2.08(5)$ & $2.08(s)$ & $2.06(\mathrm{~s})$ & $2.07(\mathrm{~s})$ & \\
\hline $2.00(\mathrm{~s})$ & $2.07(s)$ & $2.055(5)$ & $2.05(s)$ & $7,13,17-\mathrm{CH}_{3}$ \\
\hline $2.055(\mathrm{~s})$ & $2.05(\mathrm{~s})$ & $2.04(s)$ & $2.04(\mathrm{~s}) J$ & \\
\hline $1.39(d, 7.0)$ & $1.37(d, 7.0)$ & $1.39\langle d, 6.9\rangle$ & $1.21(d, 7.0)$ & $3^{\prime}-\mathrm{CH}_{3}$ \\
\hline $1.28(d, 7.4)$ & $1.28(d, 7.4)$ & $1.28(d, 7.4)$ & $1.28(d, 7.4)$ & $2-\mathrm{CH}_{3}$ \\
\hline
\end{tabular}

aThese values were recorded at $360 \mathrm{MHz}$. 
correspondence of the chemical shifts of the $2-\mathrm{CH}_{3}$, the $3^{\prime}-\mathrm{CH}_{3}$, and the C-18 vinyl protons, in conjunction with the $a-1$ and synthetic studies, conclusively proves the thioether linkage and strongly suggests its presence at the $A$ ring $3^{\prime}$-position. 20 The absence of any anomolous chemical shift differences in either the bilin or the proton resonances, in conjunction with the Edman degradation results, precludes any possible second covalent linkage between the peptides and the tetrapyrrole.

The same gross structural details are seen in the $\beta-2 T$ chromopeptide. The UV/vis spectrum and the ${ }_{H}$ NMR chemical shifts of the $C-18$ vinyl group and the $2-\mathrm{CH}_{3}$ evidence the $\mathrm{A}$ ring peptide linkage. The $3^{\prime}-\mathrm{CH}_{3}$ doublet proves the thioether attachment site at the $\mathrm{C}-3^{\prime}$ position. However, we see chemical shift differences in the $3^{\prime}-\mathrm{CH}_{3}, 3-\mathrm{H}, 3^{\prime}-\mathrm{H}, 5-\mathrm{H}$, 15-H, and 16-H resonances (Table III). In addition, the $\mathrm{\beta}-\mathrm{CH}_{2}$ resonances at 3.75-4.0 ppm are downfield from those expected for phenylalanine. ${ }^{21}$ The chemical shift differences are in the range of $0.15 \mathrm{ppm}$ and are accompanied by some coupling constant differences in the 1-2 $\mathrm{Hz}$ range. The most plausible explanation is that the phenylalanine, being four amino acid residues removed from the cysteine attachment site can coil to interact with the tetrapyrrole. As a result, the $3-\mathrm{H}, 3^{\prime}-\mathrm{H}$, and $3^{\prime}-\mathrm{CH}_{3}$ sit in the shielding region of the phenylalanine aromatic ring and are shifted upfield of their normal positions. The $\mathrm{G}-\mathrm{CH}_{2}$ position now lies in the deshielding region of the tetrapyrrole causing the observed shifts. This interaction must also slightly distort the tetrapyrrole conformation. The $5-\mathrm{H}$ shows an upfield shift and now shows a slight $(0.8 \mathrm{~Hz})$ allylic coupling to the $3-H$. In adoition, the conformationally sensitive $15-H$ and 16-H show chemical shift and coupling constant distortions.

In his synthetic studies, Gossauer has shown that the absolute 
stereochemistry at $\mathrm{C}-16$ has a dominant affect on the chiroptic properties of the phycoerythrobil in chromophore. ${ }^{20}$ That is, the $R$ configuration at C-16 causes a large positive Cotton effect in the long wavelength reg; in of the circular dichroism spectrum. The CD spectra of the four singly linked phycoerythropeptides are shown in Figure 9. A11 of the chromopeptides show the large positive Cotton effect in the $585 \mathrm{~nm}$ region concluding, for the first time, the $R$ configuration at $c-16$ for each $c=$ the singly-linked phycoerythrobilins in B-phycoerythrin's $\alpha-$ and $\beta-$ subunits.

The absolute stereochemistry at $6-2$ has been determined for isola ed phycoerythrobilin. ${ }^{20}$ Synthetic $C-2(R)$ and $C-2(S)$ phycoerythrobilin di methyl esters show very slight differences in the short wavelength region of their CD spectra with the $C-2(R)$ spectrum corresponding to that found for the tetrapyrrole isolated from C-phycoerythrin. ${ }^{20}$ Chromic acid oxidation of these materials allows the isolation of 2-(E)-ethylic ene$3(R)$-methylsuccinimide and $2-(E)$-ethylidene-3(S)-methyisuccinimide from the corresponding tetrapyrroles. The oxidation of phycoerythrobilin dimethyl ester isolated from C-phycoerythrin yields 2-(E)-ethylidene$3(R)$-methylsuccinimide, suggesting the $R$ configuration at $C-2{ }^{20}$ The presence of more than one phycoerythrobilin in these large protein complexes as well as the finding of some C-2 epinerization during the isolation of phycoerythrobilin dimethyl ester from natural sources casts some doubt on the utility of these data. 20

The CD spectra of chromopeptides $\alpha-1$ and $\beta-2 T$ show a decidedly different pattern in the $300 \mathrm{~nm}$ region than that seen for $\alpha-2$ and $t-1$. However, the $\alpha-1$ and $B-2 T$ chromopeptides contain aromatic amino acid residues whose absorption properties may influence the inter- 


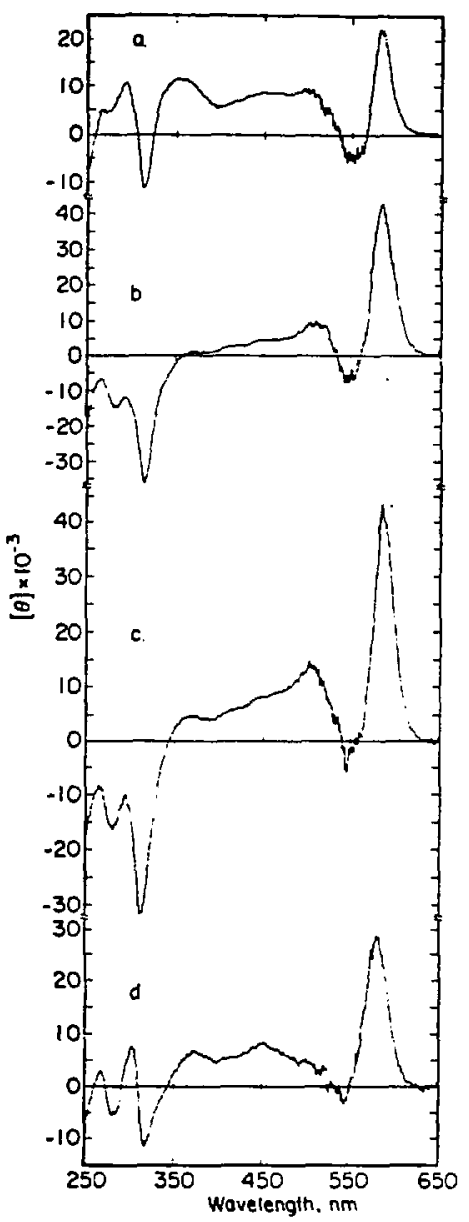

Figure 9. The circular dichroism spectra of the phycoerythrobilipeptides (a) $\alpha-1, c=54.2 \mu M$; (b) $\alpha-2, c=39.5 \mu M$; (c) $B-1, c=43.6 \mu \mathrm{M}$; (d) $B-2 T, c=29.5 \mu \mathrm{M}$ in $10 \mathrm{mM}$ aqueous TFA at $25^{\circ} \mathrm{C}$. 
pretation of these differences. Therefore, our data neither supports nor refutes any $\mathrm{C}-2$ absolute stereochemical assignments. By convention we have chosen to represent our structures as having the $C-2(R)$ configuration, with the reservation that any stereochemical proof originate from a single chromophore molecule such as those studied here. ${ }^{22}$

The assignment of the $\mathrm{C}-3$ to $\mathrm{C}-\mathrm{B}^{\prime}$ relative stereochemistry is based on the antiperiplanar elimination of the proposed sulfone group under oxidative degradation conditions of C-phycoerythrin to give the (E)-ethylidene succinimide. ${ }^{23,24}$ A detailed analysis of further evidence on this question casts doubts on the validity of any conclusions based on other data. 22 Unfortunately, the $C D$ and NMR resuits presented do not bear on this point and the $C-3(R) / C-3^{\prime}(R)$ stereochemical relationship must be adopted until further evidence is presented in proof.

Our study on the $\mathrm{C}-2 / \mathrm{C}-3$ relative stereochemistry demonstrates that the $\mathrm{C}-2 / \mathrm{C}-3$ coupling constant for cis-3,18-diethyi-1,2,3,19,21,24hexahydro-2,7,13,17-tetramethyl-1,19-dioxobilin-dipropionic acid methyl ester is $8.5 \mathrm{~Hz}$ while the trans compound exhibits a $5.4 \mathrm{~Hz}$ coupling constant (see part one of this thesis!. These compounds represent the first evidence for a trans dihydro $A$ ring in the phycocyanin series of bile pigments. ${ }^{23}$ The corresponding A ring coupling constants in the phycoerythrochromopeptides $(\alpha-1: 3.5 \mathrm{~Hz}, \alpha-2: 3.8 \mathrm{~Hz}, e-1: 3.6 \mathrm{~Hz}$, $B-2 T: 3.4 \mathrm{~Hz}$ ) are all found in the low, trans region. As the stereochemistry at $6-16$ has a profound effect on the spectroscopic properties of the phycoerythrobiitin chromophore not found in that of phycocyanobilin, these dati are suggestive, not conclusive, of the $A$ ring relative stereochemistry. For the first time, then, we are able to assign the probable stereochemistry of $C-2(R), C-3(R), C-3^{\prime}(R)$, and $C-T E(R)$ for each of the 
individual, singly-7inked Eetrapyrroles on the $\alpha$ - and $\beta$-subunits of B-phycoerythrin. 


\section{Experimental Section}

General Methods. Evaporations were done in vacuo with a Berkeley rotary evaporator. Uncorrected melting points were determined on a Buchi capillary melting point apparatus. ${ }_{1}$ NMR spectra of the synthetic peptides 1-7 were taken at $250 \mathrm{MHz}$ and chemical shifts are expressed in ppm relative to an internal TMS standard for $\mathrm{COCl}_{3}$ and relative to an external TSP standard for $D_{2} O$. The NMR spectra of the chromopeptides were taken on the University of California, Davis, $360 \mathrm{MHz}$ or $500 \mathrm{MHz}$ MMR spectrometer. High pressure liquid chromatography (HPLC) was done with an Altex system consisting of two Model 110A pumps, a Mode1 115-10 UV/vis detector, and a Model 420 microprocessor. UV/vis spectra were recorded on a Cary Model 219 spectrophotometer. Circular dichroism spectra were recorded on a Jasco J-500-C spectropolarimeter equipped with a Jasco OP-500N data processor. Elemental analyses were performed by the Analytical Laboratory, Department of Chemistry, University of California, Berkeley. Unless otherwise noted, materials were obtained from commercial suppliers and were used without further purification after verification. Dimethylformamide was distilled from $\mathrm{P}_{2} \mathrm{O}_{5}$ under reduced pressure and stored over $3 \AA$ sieves. Hydrogen fluoride was distilled from $\mathrm{CoF}_{3}$ immediately before use.

Preparation of B-Phycoerythrin. The unicellular red alga Porphyridium cruentum strain B was obtained from the Marine Biology Research Division, Scripps Institution of Oceanography, La Jolla, California, and the cells were cultured as described. ${ }^{4}$ The previously described procedure for $B-$ phycoerythrin purification was followed through the second $\left(\mathrm{NH}_{4}\right)_{2} \mathrm{SO}_{4}$ precipitation step. The protein precipitate from $100 \mathrm{~g}$ (wet weight) of 
cells in $65 \%$ saturated $\left(\mathrm{NH}_{4}\right)_{2} \mathrm{SO}_{4}$ at $25^{\circ}$ was slurried with $800 \mathrm{~mL}$ of DEAE-cellulose (Whatman DE-52, microgranular) in the same solution, and the mixture was layered onto a $200 \mathrm{~mL}$ bed of DEAE-cellulose in a $5 \mathrm{~cm}$ diameter column. The column was washed with $65 \%$ saturated $\left(\mathrm{NH}_{4}\right)_{2} \mathrm{SO}_{4}$ $50 \mathrm{mM}$ sodium phosphate, $\mathrm{pH} 7.0$ until the resin had settled to a stable bed volume. The column was then developed with decreasing concentrations of ammonium sulfate in $50 \mathrm{~m}^{4}$ sodium phoshate, $\mathrm{pH}$ 7.0. R-Phycocyanin, allophycocyanin, and b-phycoerythrin were eluted between $20 \%$ and $15 \%$ saturated $\left(\mathrm{NH}_{4}\right)_{2} \mathrm{SO}_{4}$; elution of $\mathrm{B}$-phycoerythrin was initiated with $10 \%$ saturated $\left(\mathrm{NH}_{4}\right)_{2} \mathrm{SO}_{4}$ and was completed with $0.2 \mathrm{M}$ sodium phosphate, $\mathrm{pH} 7$. B-Phycoerythrin was precipitated with $65 \%$ saturated $\left(\mathrm{NH}_{4}\right)_{2} \mathrm{SO}_{4}$, centrifuged after standing overnight at $4^{\circ} \mathrm{C}$, dissolved in the minimum volume of $20 \mathrm{mM}$ sodium phosphate, $\mathrm{pH} 7$, and dialyzed overnight at $4^{\circ} \mathrm{C}$ to equilibrium. The protein was further purified by gel filtration in tivis batches on a Sephadex G-200 column $(5 \times 50 \mathrm{~cm})$ equilibrated with $20 \mathrm{mM}$ sodium phosphate: ph 7. The B-phycoerythrin was $>95 \%$ pure after ge! $5 i$ ?tration and was free of other biliproteins.

Preparation of Phycoerythrin Peptides $\alpha-1, \alpha-2, \beta-1$, and $B-2 T$. The conditions employed for isolation and amino acid sequence studies of chromopeptides $\alpha-1, \alpha-2, \beta-1$, and $\beta-2 T$ were those described by Lunde11 et al. 6 For each chromopeptide, the eluate from the SP-Sephedex column was adsorbed onto a column of LiChrosorb $\mathrm{C}-18$, washed extensively with $10 \mathrm{mM}$ formic acid, and then eluted with $60 \%$ acetonitrile/40\% $10 \mathrm{mM}$ formic acid. The acetonitrile was removed under a stream of $\mathrm{N}_{2}$ and HPLC analysis was done using a $4.6 \times 250 \mathrm{~mm}$ U1trasphere ODS (5 $\mu \mathrm{m})$ column eluting with $22.5 \% \mathrm{CH}_{3} \mathrm{CN}$ and $77.5 \%$ of a $0.10 \mathrm{M} \mathrm{NaH}_{2} \mathrm{PO}_{4}$ buffer adjusted to pH 2.1 with concentrated $\mathrm{H}_{3} \mathrm{PO}_{4}$ (Figures 1 and 2). A flow rate of 1.0 
$\mathrm{mL} / \mathrm{min}$ was used with detection at both $200 \mathrm{~mm}$ and $550 \mathrm{~mm}$. Preparative HPLC was carried out on each peptide fraction using a $10 \times 250 \mathrm{~mm}$ UItrasphere ODS $(5 \mu \mathrm{m})$ column at a flow rate of $2.0 \mathrm{~mL} / \mathrm{min}$ and a solvent composition of $22 \% \mathrm{CH}_{3} \mathrm{CN}$ and $78 \%$ of $0.10 \mathrm{M} \mathrm{NaH}_{2} \mathrm{PO}_{4}, \mathrm{PH} 2.1$ aqueous buffer. The purification was done by multiple injections of $0.5 \mu \mathrm{mc} ;$ injection with detection at $550 \mathrm{~nm}$.

After the acetonitrile was removed by rotary evaporation at 5 torr and room temperature, the remaining aqueous solution for each chromopeptide was applied to a LiChrosorb $\mathrm{C}-18$ column $(10 \mu \mathrm{m}, 3.2 \times 250 \mathrm{~mm})$ and washed with $150 \mathrm{~mL}$ of $10 \mathrm{mM}$ aqueous TFA to desalt. Elution using $50 \%$ acetonitrile and $50 \% 10 \mathrm{mM}$ aqueous TFA then gave a $98 \%$ recovery of chromophoric material after desalting, along with $0.5 \%$ of free chromophore in a later fraction.

Sample Preparation for UV/vis and CD Spectral Analys is. Each chromopeptide fraction was concentrated to $0.5 \mathrm{~mL}$ by rotary evaporation and diluted with $10 \mathrm{mM}$ aqueous TFA to appropriate concentrations for UV/vis and CD spectral analyses (Figures 3,4 , and 9 ).

Sample Preparation for NMR Spectral Analysis. Each chromopeptide fraction in $10 \mathrm{mM}$ aqueous TFA was concentrated to $0.5 \mathrm{~mL}$ by rotary evaporation and djluted with $10 \mathrm{~mL}$ of $10 \mathrm{mM}$ trifluoroacetic acio in $99.8 \% \mathrm{D}_{2} \mathrm{O}$ (made by adding $50 \mu \mathrm{mol}$ of trifluoroacetic anhydride to $10 \mathrm{~mL}$ of $\mathrm{D}_{2} \mathrm{O}$ ). The solution was concentrated to $0.5 \mathrm{~mL}$ as before and this same procedure was repeated three times, twice utilizing $1.0 \mathrm{~mL}$ of $10 \mathrm{mM}$ TFA in $99.96 \% \mathrm{D}_{2} \mathrm{O}$, and once using $1.0 \mathrm{~mL}$ of $10 \mathrm{mM}$ TFA in $99.996 \%$ $D_{2}$. Finally, the NMR samples were prepared in $1.0 \mathrm{~mL}$ of $10 \mathrm{mM}$ TFA in 99.996\% $\mathrm{D}_{2} \mathrm{O}$ at the following concentrations: $\alpha-14.4 \mathrm{mM}, \alpha-25.2 \mathrm{mM}$, $B-14.2 \mathrm{mM}$, and $B-2 T 2.6 \mathrm{mM}$. 
N-tert-Butyloxycarbony]-0-benzyl-L-tyrosyl-N $N^{E}-n i t r o-L$-arginine

Benzyl Ester (3). N-tert-Butyloxycarbonyl-0-benzyl-L-tyrosine (1) 9,10 $(0.26 \mathrm{~g}, 0.70 \mathrm{mmol})$ was dissolved in $2 \mathrm{~mL}$ of DMF and cooled to $-20^{\circ} \mathrm{C}$ before $80 \mu \mathrm{L}(0.73 \mathrm{mmol})$ of $N$-methylmorpholine and $95 \mu \mathrm{L}(0.73 \mathrm{mmol})$ of isobutyl chloroformate were added. After a twenty minute activation, $\mathrm{N}^{\varepsilon}$-nitro-L-arginine benzyl ester $(2)^{12}(0.27 \mathrm{~g}, 0.87 \mathrm{mmol})$ in $2 \mathrm{~mL}$ of DMF was added and the mixture stirred for $3 \mathrm{~h}$ at $-20^{\circ} \mathrm{C}$. The solvert was removed by rotary evaporation at 0.1 torr and room temperature and the residue was taken up in $25 \mathrm{~mL}$ of ethyl acetate and $10 \mathrm{~mL}$ of $\mathrm{H}_{2} \mathrm{O}$. The organic layer was washed successively with $10 \mathrm{~mL}$ of $1 \mathrm{M}$ citric acid, 10 $\mathrm{mL}$ of water, $10 \mathrm{~mL}$ of aqueous saturated sodium bicarbonate, $10 \mathrm{~mL}$ of water, and $10 \mathrm{~mL}$ of aqueous saturated sodium chloride, then dried over $\mathrm{Na}_{2} \mathrm{SO}_{4}$, filtered, and evaporated to give an oily product which was purified by HPLC. A LiChrosorb $5 i-60$ column $(10 \mu \mathrm{m}, 10 \times 250 \mathrm{~mm})$ was used with elution with $2 \%$ methanol in chloroform at $2.8 \mathrm{~mL} / \mathrm{min}$, detection at $280 \mathrm{~nm}$, and multiple injections of $10 \mathrm{mg}$ each. A single product with a retention time of 5.1 minutes was present and was collected. Solvent was evaporated to give a clear oil which was crystallized from ethy acetate/ether: $0.17 \mathrm{~g}\left(37_{\%}^{\circ}\right)$ of 3 as an amorphous white powder, mp $156-157^{\circ} \mathrm{C}$; NMR $\left.\left(\mathrm{CDCl}_{3}\right) 1.37\left(\mathrm{~s}, 9 \mathrm{H},\left(\mathrm{CH}_{3}\right)_{3} \mathrm{C}\right)\right), 1.5-1.9\left(\mathrm{~m}, 4 \mathrm{H}, \operatorname{Arg} \mathrm{B}, \gamma-\mathrm{CH}_{2}\right), 2.95$ $\left(\mathrm{m}, 1 \mathrm{H}, \operatorname{Tyr} \mathrm{B}-\mathrm{CH}_{2}\right), 3.20\left(\mathrm{~m}, 2 \mathrm{H}\right.$, Arg $\left.\delta-\mathrm{CH}_{2}\right), 3.50\left(\mathrm{~m}, \mathrm{H}, \operatorname{Tyr} \mathrm{B}-\mathrm{CH}_{2}\right)$, $4.30(\mathrm{~m}, \mathrm{IH}, \operatorname{Arg} \alpha-\mathrm{CH}), 4.60(\mathrm{~m}, \mathrm{H}, \mathrm{Tyr} \alpha-\mathrm{CH}), 5.00\left(\mathrm{~s}, 2 \mathrm{H}, \mathrm{PhOCH}_{2} \mathrm{Ph}\right)$, 5.15 (s, $\left.2 \mathrm{H}, \mathrm{CO}_{2} \mathrm{CH}_{2} \mathrm{Ph}\right), 6.90$ (d, $\left.2 \mathrm{H}, \operatorname{Tyr} \mathrm{Ar}-3,5 \mathrm{H}\right), 7.10$ (d, $2 \mathrm{H}$, Tyr Ar-2,6H), $7.35(\mathrm{~m}, 1 \mathrm{OH}, \mathrm{Ar})$. Anal. Calcd for $\mathrm{C}_{34} \mathrm{H}_{42} \mathrm{~N}_{6} \mathrm{O}_{8} \mathrm{H}_{2} \mathrm{O}$ : $\mathrm{C}, 60.0 ; \mathrm{H}, 6.5 ; \mathrm{N}, 12.4$. Found: $\mathrm{C}, 60.4 ; \mathrm{H}, 6.2 ; \mathrm{N}, 12.4$. N-tert-Butyloxycarbonyl-S-acetami dome thy 1 -L-cysteinyl-0-benzylL-tyrosyl-N $N^{E}-n i t r o-L-a r g i n i n e ~ B e n z y l$ Ester $(6)$. The Boc group was 
removed from dipeptide 3 (96 $\mathrm{mg}, 0.14 \mathrm{mmol}$ ) by dissolution in $25 \mathrm{~mL}$ of dioxane previously saturated with $\mathrm{HCT}$. After 30 min at room temperature, the volatjle components were evaporated and the residue was triturated with anhydrous ether to give $79 \mathrm{mg}(94 \%)$ of the HCl salt $\underset{5}{5}$ as a white powder. N-tert-Butyloxycarbonyl-5-acetamidomethyl-L-cysteine $(4)^{8,9}(46.2$ $\mathrm{mg}, 0.16 \mathrm{mmol}$ ) was dissolved in $1.5 \mathrm{~mL}$ of $\mathrm{DMF}$ and cooled to $-25^{\circ} \mathrm{C}$, and $21 \mu \mathrm{L}$ (0.16 mmol) of isobutyl chioroformate and $17.4 \mathrm{\mu L}(0.16 \mathrm{mmol})$ of $\mathrm{N}$-methylmorpholine were added. After a 25 min activation time, a solution of the dipeptide salt $(\underset{5}{5}, 79 \mathrm{mg}, 0.13 \mathrm{mmol})$ anj $14.5 \mu \mathrm{L}$ (0.13 mmol) of $\mathrm{N}$-methylmorphol ine in $1.5 \mathrm{~mL}$ of DMF was added. After $3 \mathrm{~h}$ at $-25^{\circ} \mathrm{C}$, the 50 lvent was evaporated and isolation proceeded as described for 3 above. HPLC purification was done under the same conditions giving one peak, $R_{t} 4.2$ min: yield, $76 \mathrm{mg}, 70 \%$, of 6 as a clear foam; $\operatorname{NMR}\left(\mathrm{CDCl}_{3}\right) 1.40\left(\mathrm{~s}, 9 \mathrm{H},\left(\mathrm{CH}_{3}\right)_{3} \mathrm{C}\right), 1.55-1.85\left(\mathrm{~m}, 4 \mathrm{H}\right.$, Arg $\left.\mathrm{B}, \gamma-\mathrm{CH}_{2}\right)$, $2.00\left(\mathrm{~s}, 3 \mathrm{H}, \mathrm{COCH}_{3}\right), 2.75\left(\mathrm{~m}, 2 \mathrm{H}, \mathrm{Cys} \mathrm{B}-\mathrm{CH}_{2}\right), 2.90\left(\mathrm{~m}, \mathrm{HH}, \mathrm{Tyr} \mathrm{B}-\mathrm{CH}_{2}\right)$, $3.04\left(\mathrm{~m}, 2 \mathrm{H}, \operatorname{Arg} \delta-\mathrm{CH}_{2}\right), 3.20\left(\mathrm{~m}, \mathrm{HH}\right.$, Tyr $\left.8-\mathrm{CH}_{2}\right), 4.20(\mathrm{~m}, \mathrm{IH}$, Arg $\alpha-\mathrm{CH})$, $4.35(\mathrm{~m}, \mathrm{lH}, \mathrm{Tyr} \alpha-\mathrm{CH}), 4.55\left(\mathrm{~m}, 3 \mathrm{H}\right.$, Cys $\left.\alpha-\mathrm{CH}_{3} \mathrm{SCH}_{2} \mathrm{NH}\right), 5.00(\mathrm{~s}, 2 \mathrm{H}$, $\left.\mathrm{PhOCH}_{2} \mathrm{Ph}\right), 5.13\left(\mathrm{~s}, 2 \mathrm{H}, \mathrm{CO}_{2} \mathrm{CH}_{2} \mathrm{Ph}\right), 6.87(\mathrm{~d}, 2 \mathrm{H}, \mathrm{Tyr} \mathrm{Ar}-3,5 \mathrm{H}), 7.10$ $(d, 2 \mathrm{H}, \operatorname{Tyr} \mathrm{Ar}-2,6 \mathrm{H}), 7.35(\mathrm{~m}, 10 \mathrm{H}, \mathrm{Ar})$. Anal. Calcd for $\mathrm{C}_{40} \mathrm{H}_{52} \mathrm{~N}_{3} \mathrm{O}_{10} \mathrm{~S} \mathrm{H}_{2} \mathrm{O}: \mathrm{C}, 56.2 ; \mathrm{H}, 6.4 ; \mathrm{N}, 13.1$. Found: C, 56.5; H, 6.2; $N, 13.2$.

\section{S-Acetamidomethyl-L-cysteinyl-L-tyrosyl-L-arginine}

Ditrifluoroacetate (7). The protected tripeptide $6(54 \mathrm{mg}, 64 \mu \mathrm{mol})$ along with $70 \mu \mathrm{L}$ of anisole was treated with anhydrous hydrogen fluoride at $0^{\circ} \mathrm{C}$ for 30 min after which the $\mathrm{HF}$ was evaporated. The residue was taken up in water $(7 \mathrm{~mL})$, washed with ethyl acetate $(2 \times 3 \mathrm{~mL})$, and iyophilized. The residue was purified by HPLC on a Spherisorb ODS column $(10 \mu \mathrm{m}, 10 \times$ 
$250 \mathrm{~mm}$ ) using $25 \% \mathrm{CH}_{3} \mathrm{CN}$ and $75 \% 10 \mathrm{~min}$ açueous TFA as eluent at a flow rate of $4 \mathrm{~mL} / \mathrm{min}$ with detection at $270 \mathrm{~nm}$ and multiple injections of $5 \mathrm{mg}$ each. One minor and one major $\left(R_{t}=10 \mathrm{~min}\right)$ product were seen. The major peak was collected and lyophilized to give $22 \mathrm{mg}(46 \%)$ of the tripeptide bis(trifluoroacetate) 7. The NMR sample was prepared as described for chromopeptides and was determined at $5.0 \mathrm{mM}$ in $10 \mathrm{mM}$ TFA in $\mathrm{D}_{2} \mathrm{O}$ (Figure 4 ). 


\section{Acknowledgement}

This research was supported in part by the Division of Biological Energy Conversion and Conservation $(D O E)$, the National Institute of General Medical Sciences, DHHW, grant GM 28994, and National Science Foundation grant NSF PCM 82-08158. The $360 \mathrm{MHz}$ and $500 \mathrm{MHz}$ NMR studies were carried out at the University of California, Davis, MRR Facility, under the auspices of National Science Foundation Grant CHE 79-04832. 


\section{References}

(1) Giazer, A.N. "The Biochemistry of Plants"; Hatch, M.D.; Soardmann, N.K., Eds.; Academic Press: New York, 1981; Vo1. 8, p 51.

(2) 0 Carra, P.; 0 hEocha, C. "Chemistry and Biochemistry of Plant Pigments"; Goodwin, T.W., Ed.; Academic Press: New York, 1976; Vol. 1, p 328.

(3) Gantt, E.; Lipschultz, C.A. Biochemistry $\underset{\sim 7}{1974}, \underline{13}, 2960$.

(4) Glazer, A.N.; Hixsoit, C.S. J. Biol. Chem. 1977, 252, 32.

(5) Abad-Zapatero, C.; Fox, J.L.; Hackert, M.L. Biochem. Biophys. Res. Commun. 1977, 78, 266.

(6) Lunde17, D.J.; Glazer, A.N.; DeLange, R.J.; Brown, D.M. J. Biol. Chem. 1983, submitted.

(7) Anderson, G.W.; Zimmerman, J.E.; Callahan, F.M. J. Am. Chem. Soc. $1967,89,5012$.

(8) Veber, D.F.; Milkowski, J.D.; Varga, S.L.; Denkewalter, R.G.; Hirschmann, R. J. Am. Chem. Soc. 1972, 94, 5456.

(9) Moroder, L.; Hallett, A.; Wunsch, E.; Keller, O.; Wersin, G. Hoppe-Seyier's Z. Physiol. Chem. 1976, 357, 1651.

(10) Wunsch, E.; Fries, G.; Zwick, A. Chem. Ber. 1958, 91, 542.

(11) Hayakawa, T.; Fujiwara, Y.; Noguchi, J. Bull. Chem. Soc. Japan $1967,4 \underline{40}, 1205$.

(12) Zervas, L.; Winitz, M.; Greenstein, J.P. J. Org. Chem. 1957, 22, 1515.

(ij) Sakakibara, S.; Shimonishi, Y. Bull. Chem. Soc. Jacan, 1965, 33, 1412.

(14) Lagarias, J.C.; Glazer. A.N.; Rapoport, H. J. Am. Chem. Soc. 1979, 101,5030 .

(15) Lagarias, I.C.; Rapoport, H. J. Am. Chem. SoC. 1980, 102, 4821. 
(16) McDonagh, A. "The Porphyrins"; Dolphin, D., Ed.; Acadenic Press: New York, 1979; Vol. III A, p 293.

(17) Crespi, H.L.; Katz, J.J. Phytochemistry 1969, 8, 759.

(18) Chapman, D.J.; Cole, W.J.; Siegelman, H.W. J. Am. Chem. Soc. 1967, 89, 5976 .

(19) Gossauer, A. Klahr, E. Chem. Ber. 1979, 112, 2243.

(20) Gossauer, A. Weller, J.-P. J. Am. Chem. Soc. 1978, 100, 5928.

(21) Wuthrich, K. "NMR in Biological Research: Peptides and Proteins"; American Elsevier: New York, 1976; p 42.

(22) Gossauer, A. Tetrahedron $\underset{\sim \sim \sim}{1983}, 39,1933$.

(23) Schoch, S.; Klein, G.; Linsenmeier, U.; Rudigar, W. Justus Liebigs Ann. Chem. 1976, 549.

(24) Klein, G.; Rudiger, W. Justus Liebigs Ann. Chem. 1978, 207. 


\section{Part Three}

Bilin Attachment Sites in the $\alpha$ and $B$ subunits of B-Phycoerythrin: Structural Studies on a Doubly Peptide-Linked Phycoerythrobilin

\section{Abstract}

A bilin-containing fragment, B-3T, from Porphyridiun cruentum B-phycoerythrin produced by cleavage with trypsin and thermolys in was shown to have the structure:

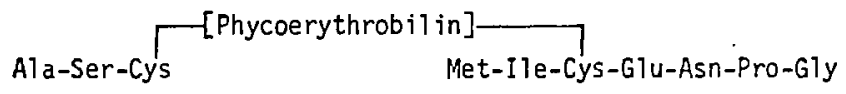

secondary ion mass spectrometry of this bilin-peptide yielded a protonated molecular ion of 1629 mass units corresponding to that predicted from the composition of the fragment, and indicated that the heptapeptide is linked to ring $A$ and the tripeptide to ring $D$. NMR spectra provided definitive evidence for a thioether linkage at the $\mathrm{C}-3^{\prime}$ of ring $A$ and a second thioether linkage at $C-18^{\prime}$ of ring $D$ of the bilin. This is the first documented report of a bilin linked through two thioether covalent bonds to a poiypeptide. 
The phycobiliprotein B-phycoerythrin from the unicellular red alga Porphyridium cruentum has the subunit composition $(\alpha \beta)_{6} \gamma{ }^{1-3}$ The $\alpha$ and B subunits carry only phycoerythrobilin chromophores whereas the $\gamma$ subunit carries both phycoerythrobilins and phycourobilins. ${ }^{2}$ All of the phycoerythrobilin groups on the $\alpha$ and $\beta$ subunits can be accounted for in five tryptic peptides each carrying one phycoerythrobilin, two derived from the $\alpha$ subunit and three from the $\beta$ subunit. ${ }^{4}$ In four of these peptides, the bilin is attached by a single thioether linkage to a cysteinyl residue, however, in the fifth peptide, $\beta-3$, sequence analys is showed the presence of two thioether link jes to the bilin from cysteinyl residues ten residues apart in the linea" sequence. The sequence of $\beta-3$ was determined to be

$$
\begin{aligned}
& \text { Leu-Asp-Ala-Va 1-Asn-Ser-Ile-Val-S _r-Asn-Ala-Ser-Cys*-Met- } \\
& \text { Val-Ser-Asp-Ala-Val-Ser-G1y-Het-[1e-Cys*-Glu-Asr-Pro-G1y- } \\
& \text { Leu-I1e-Ser-Pro-G1y-G1y-Asn-Cys-Tyr-Thr-Asn-Arg }
\end{aligned}
$$

where the asterisks mark the sites of bilin attachrnent. ${ }^{4}$

The finding of this doubly- inked phycoerythrobilin was unexpected. Previous studies of phycoerythrin-peptides from cyanobacterial C-phyccerythrins had shown a single thioether linkage to the bilin in each peptide. 5,6

Thermolysin digestion of the $\beta-3$ chromopeptide gives a smaller B-3T fragment. From amino acid sequence analysis, the structure of this fragrient was deduced to ve:

$$
\text { Ala-Ser-Cys }[-[\text { Phycoerythrobilin }]-[\text { Met-Ile-Cys-Glu-Asn-Pro-Gly }
$$

The UV/Vis spectra showed that no alteration of the chromophore took place during the tharmolysin cleavage. ${ }^{4}$ The loss of the claracteristic 
red color of the tetrapyrrole during Edman degradation was seen only as both indicated cysteine residues were removed, suggesting the doubly-linked nature of this chromophore.

The secondary ion mass spectrum (SIMS) of the bilin-peptide B-3T was recorded using a cesium ion primary beam. ${ }^{7}$ The high mass region (Table I) shows a strong molecular ion peak along with a number of confirming salt adducts. As shown in Tables I and II, each mass is actually represented by a cluster of peaks. Generally, the primary mass is the unprotonated species. An exception to this is seen in Table I where the

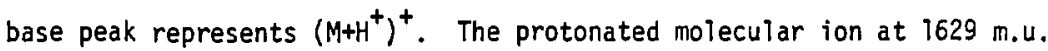
is the result of raunding up (above 0.59 ) of the exact mass measured at $1628.94 \mathrm{~m} . \mathrm{u}$. From compositional data, the calculated exact mass of $\left(M+H^{+}\right)^{+}$is $1628.68 \mathrm{~m} . \mathrm{u}$. , and this agreement is within the accuracy of the instrumentation ( \pm 0.3 m.u.).

In addition to the molecular ion information, the positive SIMS showed a number of useful fragments. Small peaks, at 1497 m.u. and 1519 m.u. , represent (M-Met) ${ }^{+}$and $\left(M-M e t-H^{+}+N^{+}\right)^{+}$, respectively, resultin: from the loss of the amino-terminal methionyl residue from the heptapeptide portion of $B-3 T .^{8,9}$ A small peak at $1349 \mathrm{~m} . \mathrm{u}$. attests to the presence of the (M-tripeptide) ${ }^{+}$fragment resulting from the ioss of Ala-Ser-Cys. Hovever, all of the majer fragments result from the loss of the heptapeptide moiety from B-3T (Table II). The heptapeptide itself is seen in the 763,785 , and $801 \mathrm{~m} . u$. peak series. The most abundant fragment, $743 \mathrm{m.u}$, is derived from the heptapeptide by the loss of $\mathrm{CH}_{2} \mathrm{CO}_{2}-$ from the $\mathrm{C}$-terminal glycine leaving a termina? amide-containing fragment. ${ }^{8}$ The other two major fragments in the low mass fragmentation region contain the bilin as (tetrapyrrole $+\mathrm{H}^{+}$) ${ }^{+}$at 587 m.u. and as 
Table 1. High Mass Region of the Positive Secondary Ion Mass Spectrum of the $B-. \quad \because ;$ coerythrobilipeptide.

Mass

1628

1629

1650

1666

1672

1688

1742

1764
Relative Intensity $\langle \%\rangle$

79

100

60

36

23

15

14

14
Assignment

$$
\begin{aligned}
& \mathrm{Ni}^{+} \\
& \left(\mathrm{M}+\mathrm{H}^{+}\right)^{+} \\
& \left(\mathrm{M}-\mathrm{H}^{+}+\mathrm{Na}^{+}\right)^{+} \\
& \left(\mathrm{M}-\mathrm{H}^{+}+\mathrm{K}^{+}\right)^{+} \\
& \left(\mathrm{M}-2 \mathrm{H}^{+}+2 \mathrm{Na}^{+}\right)^{+} \\
& \left(\mathrm{M}-2 \mathrm{H}^{+}+\mathrm{Na}^{+}+\mathrm{K}^{+}\right)^{+} \\
& (\mathrm{M}+\mathrm{TFA})^{+} \\
& \left(\mathrm{M}-\mathrm{H}^{+}+\mathrm{Na}^{+}+\mathrm{TFA}\right)^{+}
\end{aligned}
$$


Tabie II. Low Mass Fragmentation Region of the Positive Secondary Ion Mass Spectrum of the $\beta-3 T$ Phycoerythrobilipeptide.

\begin{tabular}{lll} 
Moss & Relative Intensity $(\%)^{\text {a }}$ & Assignment \\
\hline 866 & 20 & $\begin{array}{l}\left(\text { M - heptapeptide }+\mathrm{H}^{+}\right)^{+} \\
801\end{array}$ \\
74 & $\begin{array}{l}\left.\text { (Heptapeptide }-\mathrm{H}^{+}+\mathrm{K}^{+}\right)^{+} \\
785\end{array}$ & (Heptapeptide $\left.-\mathrm{H}^{+}+\mathrm{Na}^{+}\right)^{+}$ \\
763 & 25 & (Heptapeptide $\left.+\mathrm{H}^{+}\right)^{+}$ \\
743 & 12 & (Heptapeptide $\left.-\mathrm{CH}_{2} \mathrm{CO}_{2}^{-}+\mathrm{K}^{+}\right)^{+}$ \\
727 & 94 & (Heptapeptide $\left.-\mathrm{CH}_{2} \mathrm{CO}_{2}^{-}+\mathrm{Na}^{+}\right)^{+}$ \\
587 & $i 2$ & (Tetrapyrrole $\left.+\mathrm{H}^{+}\right)^{+}$
\end{tabular}

${ }^{a}$ The base peak was $115 \mathrm{~m} . \mathrm{u}$. (glycerol $\left.-\mathrm{H}^{+}+: \mathrm{l}^{+}\right)^{+}$derived from the glycerol matrix. 
(M-heptapeptide $\left.+\mathrm{H}^{+}\right)^{+}$at 866 m.u.

Calibration problems prevented full interpretation of the negative SIMS. However, the only major peaks seen corresponded to the molecular ion and the free heptapeptide.

The molecular ion information presented above give excellent confimation of structure 1. In conjunction with the MMR data (see below). the doubly-linked nature of this bilin is conclusively demonstrated. The saturation at $\mathrm{C}-15,16$ and the unsaturation at $\mathrm{c}-17,18$ are further do imented. Also, the mass spectrum precludes any poss:ble ester linkage as is often postulated for bilin-peptides. 10

The fragmentation gives additional information about the peptide moieties. Losses of methionine and glycine establish their positions as $\mathrm{N}$ - and $\mathrm{C}$-terminal, respectively, in the peptide moieties. A7though computer analysis of some of the minor peaks might give further sequerce data, this information was more readily available by other methods. ${ }^{4}$ The fragmentition appears to be indicative of the relative lability of the two peptide-tetrapyrrole linkages. The major $866 \mathrm{~m} . \mathrm{u}$. fragment indicates the loss of the heptapeptide through a two electron process with additional loss of hydrogen generating a double bond in the tetrepyrrole. The $587 \mathrm{~m} . \mathrm{u}$. peak for the protonated tetrapyrrole and the minor $1349 \mathrm{~m} \cdot \mathrm{u}$. peak for the loss of the tripeptide imply that the tripeptide is cleaved inrough this same type of mechanism.

This is the first report of a $D$ ring thioether attachment. Mechanistic studies of tetrapyrrole-peptide cleavages in solution show that the rather facile thioether el imination at ring $A$ involves the loss of the tertiary allylic hydrogen at $C-3$, perhaps assisted by favorable stereochemistry. 11 such data, of course, do not exist for el imination 


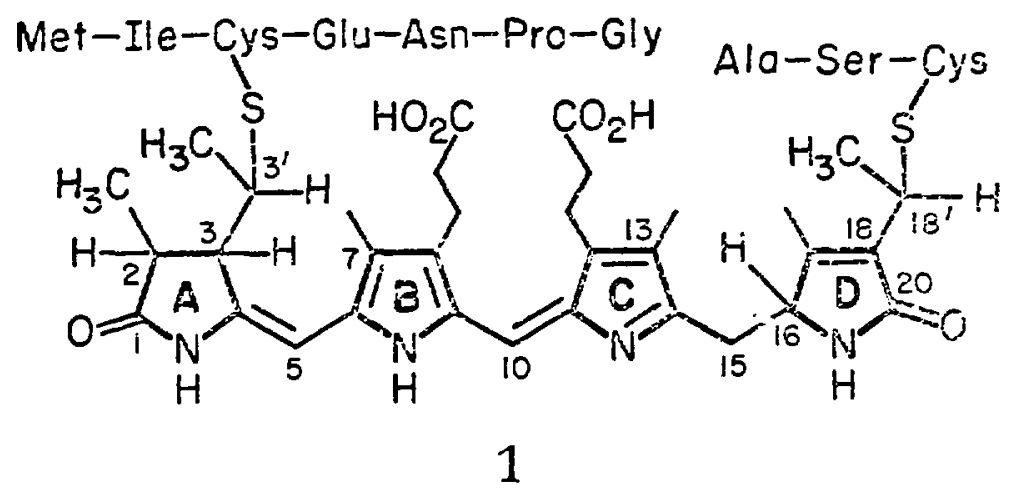

B-3T Phycoerythrobil ipeptide 
of the thioether at ring $D$. However, the structure as assigned in 1 indicates that such an elimination should be more difficult at ring $\mathbf{C}$ than at ring $A$. Thus elimination of the $D$ ring thioether group could involve any of threc hydrogens: (a) a primary homoallylic hydrogen from the $\mathrm{C}-18^{\prime}-\mathrm{CH}_{3}$, (b) a primary allylic hydrogen from the $\mathrm{C}-17-\mathrm{CH}_{3}$, or (c) the tertiary allylic hydrogen at $\mathbf{C}-16$. Processes involving (a) or (b) would be less favorable than one involving (c). Even the latter case, however, requiring a 1,4-elimination and resulting in a less conjugated system, appears less faciie than elimination from ring $A$.

At this point, our evaluation of the relative ease of elimination at the two sites is conjectural and will be resolved only by direct comparison of reactivity with specific, authentic structures. However, the striking dominance of all major fragments originating from the loss of the reptapeftide clearly demonstrates that there is a significart difference between the two modes of e? imination. We find this difference is best accommodated by the assignment of structure I.

The NMP. spectrum of the biliri-peptide $8-3 T$ in 10 mM TFA in $D_{2} 0$ is shown in Figure 1. This solvent was chosen because the chromophare has good stability in it and because its acidic nature precludes the existence of interconverting prototropic isomers. The peptice resonances show very good resolution while the tetrapyrrolic sigilals show severe line brondening, especially the $5-H$ and $10-H$ resonances. The same phenolienon has been seen to a lesser degree in frevious MMR studies of bilin-peptides. ${ }^{12}$ Evidently the peptice portions, while themselves free to extenc into solution, provide steric constraints on the conformationa? fiexibility of the bilin.

The Tine troadening in conjunction vitir overlapping sigitels pre- 


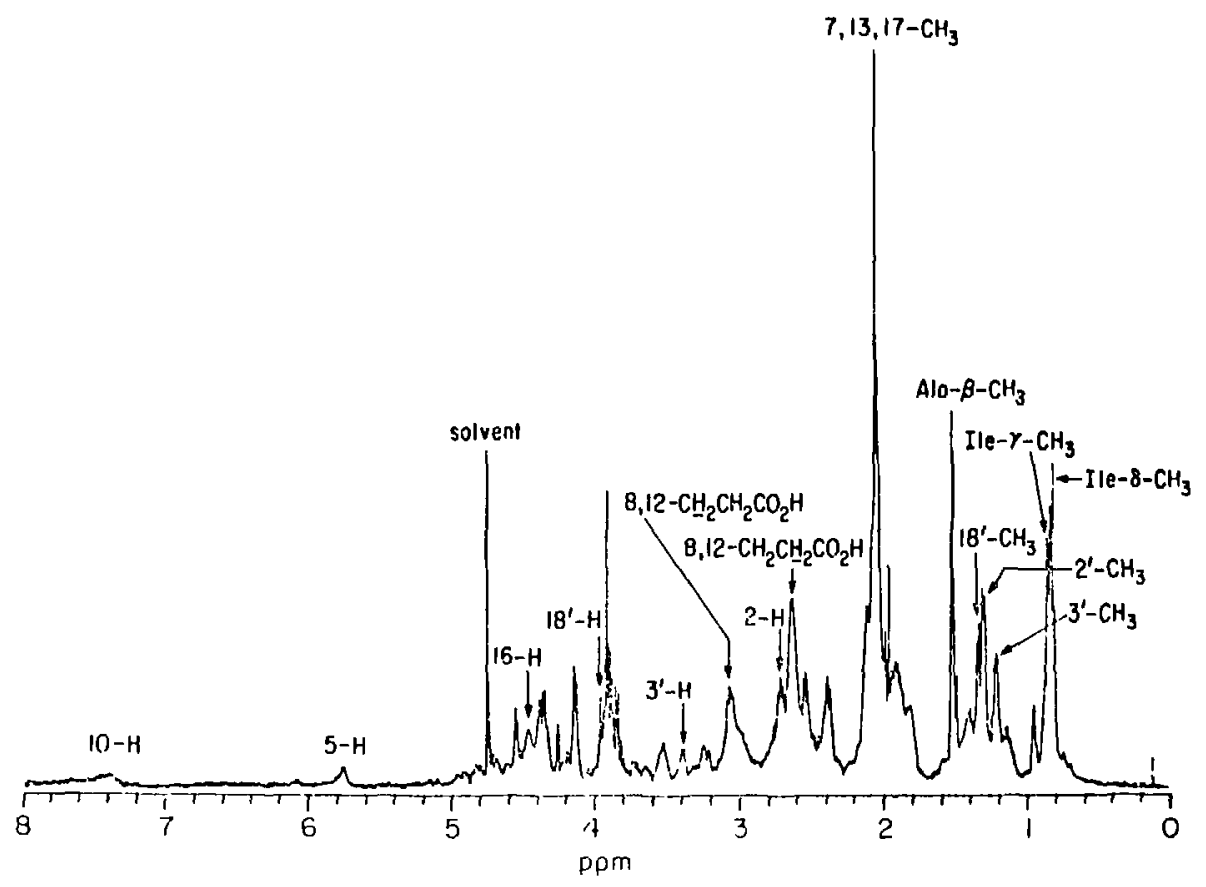

Fijure 1. The $500 \mathrm{MHz}{ }^{T_{H}}$ NMR spectrum of the phycoerythrobilipeptiae $B-3$ T $(c=2.2$ nill $)$ in 10 nM TFA in $D_{2} \mathrm{O}$ at $25^{\circ} \mathrm{C}$. 
cludes complete assignment of all of the tetrapyrrolic-peptide resonances. In particular, the $3-\mathrm{H}$ and $15-\mathrm{H}$ protons could not be assigned even by difference decoupling. The iine broadening aiso prevented the assessment of the $2-11 / 3-H$ coupling constart and no relative stereochemical information sculd be extracted.

The ${ }^{1} H$ MMR assignments for the bilin moiety, together with those for the phycoerythrobilin bearing $\alpha-1$ tripeptide 2, Cys (PEB)-Ty: -Arg (see part two of this thesis), are shown in Tabie III. The general substitution pattern of the tetrapyrrole is asslined by analogy to other studies on phycoerythrobilins. ${ }^{13}$ Comparison with tre data on the trupeptide permite straightforward assigninent of most of the bi] in resonances. The key to the important assignments in the $A$ and $D$ rings lies in the upfield (0.7-1.6 ppm) region. Decoupling experiments dlow us to assign the isoleucine s-mathyl triplet at $0.81 \mathrm{jpm}$ and the isoleucine $\gamma$-methyl doublet at $0.84 \mathrm{ppm}$. The alanine methy! doublet appears at $1.51 \mathrm{ppm}$. These assignments leave three broadened (relative to the pepticio resonances) doublets attributable to the bilir. The doublet at $1.29 \mathrm{ppm}$ is coupled to the proton at $2.7 \mathrm{ppm}$. These vaiues correspond to resonances assigned to $2-\mathrm{CH}_{3}$ and $2-\mathrm{H}$ in the tripeptide. Such agreenent wouTd be expected because the 2-position is least. influenced by conformational effects.

The two remaining upfield doublets provide direct eviaence for the two thioether linkages at the $3^{\prime}$ - and $18^{\prime}$-positions. The methyi doublet lying further do:snfiold is coupled to the proton at $3.9 \mathrm{ppm}$. The presence of three aromatic methyl groups in the $2.04 \mathrm{ppm}$ region and the absence of another methyl doublet shows that the $C-17 / \mathrm{C}-1 \mathrm{~S}$ double b.nd is still intact. As the 3.a ppan protur is allylic ard 


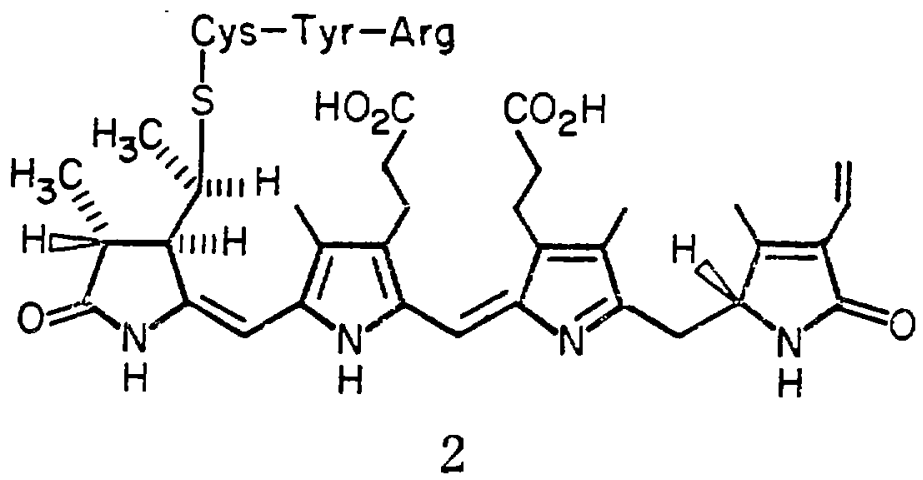

$\alpha-1$ Phycoerythrobilitripeptide 
Table III. $I_{H}$ NMR Assignments for the Bilin Moiety of Phycoerythrobilipeptide $\beta_{-}$- 1 T and the $\alpha_{-1}$ Phycoerythrobilitripeptide 2 in $10 \mathrm{mM}$ TFA in $\mathrm{D}_{2} \mathrm{O}$ at $25^{\circ} \mathrm{C}$.

\begin{tabular}{|c|c|c|}
\hline 1 & $\underline{2}^{a}$ & assignment \\
\hline $7.4(5)$ & $7.34(s)$ & $10-\mathrm{H}$ \\
\hline$b$ & $6.37(d d, 11.7,17.9)$ & $19-\mathrm{H}_{\mathrm{a}}$ \\
\hline $5.75(\mathrm{~s})$ & $5.86(\mathrm{~s})$ & $5-H^{\circ}$ \\
\hline b & $5.82(\mathrm{dd}, 1.4,17.9)$ & $18-\mathrm{H}_{b}$ \\
\hline b & $5.40(\mathrm{dd}, 1.4,11.7)$ & $18-H_{x}$ \\
\hline $4.46(\mathrm{~m})$ & $4.40(d d, 4.2,7.9)$ & $16-H^{n}$ \\
\hline $3.9(\mathrm{~m})$ & b & $18^{\prime}-\mathrm{H}$ \\
\hline $3.40(\mathrm{~m})$ & $3.44(\mathrm{~m}, 3.0,7.0)$ & $3^{\prime}-\mathrm{H}$ \\
\hline c & $3.30(d d, 4.2, ? 4 . ?)$ & $15-\mathrm{H}$ \\
\hline c & $3.10(m, 3.0,3.5)$ & $3-H$ \\
\hline $3.05(\mathrm{~m})$ & $3.07(\mathrm{~m})$ & $8,12-\mathrm{CH}_{2} \mathrm{CH}$ \\
\hline c & $2.93(\mathrm{~m}, 7.9,14.1)$ & $15-\mathrm{H}$ \\
\hline $2.7(\mathrm{~m})$ & $2.69(\mathrm{~m}, 3.5,7.4)$ & $2-\mathrm{H}$ \\
\hline $2.6(\mathrm{~m})$ & $2.64(\mathrm{~m})$ & $8,12-\mathrm{CH}_{2} \mathrm{CH}$ \\
\hline $2.05(\mathrm{~s})$ & $2.08(\mathrm{~s})$ & \\
\hline $2.04(s)$ & $2.06(s)$ & $7,13,17-\mathrm{CH}_{3}$ \\
\hline $2.03(\mathrm{~s})$ & $2.055(\mathrm{~s})$ & \\
\hline $1.33(d, 7.2)$ & b & $18^{\prime}-\mathrm{CH}_{3}$ \\
\hline $1.29(d, 6.6)$ & $1.28(d, 7.4)$ & $2-\mathrm{CH}_{3}$ \\
\hline $1.21(d, 5.5)$ & $1.39(d, \ldots 0)$ & $3^{\prime}-\mathrm{CH}_{3}$ \\
\hline
\end{tabular}

Data from part two of this thesis.

besignment is not applicable as th s functionality differs in this compound.

${ }^{C}$ Overlapping resonances and line broadening prevent assignment. 
therefore expected to be shifted further downfield, we assign this resonance to the $\mathrm{C}-1 \mathrm{~B}^{\prime}$ proton. The $\mathrm{C}-3^{\prime}$ methyl, then, appears at $1.21 \mathrm{ppm}$ and is coupled to the $\mathrm{C}-3^{\prime}-\mathrm{H}$ at $3.4 \mathrm{ppm}$. The difference in the chemical shift of the $\mathrm{C}-3^{\prime}$ methy $(0.18$ ppm upfield from that in the tripeptide) along with the severely broadened nature of the signal indicate that it is sterically constricted in the shielding region of the chromophore.

The absence of attributable vinyl resonances provides confimatory evidence of the second thioether liniage at C-18'. Structure $I$ is drawn with the peptide unit originally closer to the amino-terminus of the protein as bound to ring $D$ since the mass spectrai data supports this assignment. The finding of a doubly-linked phycoerythrobilin mandates the re-evaluation and indicates the limitations of other siudies of linleages to bilins. ${ }^{14}$ our study of the phycoerythrobilin-pepicides from the $a$ and $B$ subunits of $P$. crueritum B-phycoerythrin definitively sicws that four of the bilins are singly-liniked through the $A$ ring and the remaining bilin is doubly-linked. ${ }^{13}$ The existence of a bilin singiylinked through the $D$ ring has never been proven but seems likely in light of the present results. The biological significance of these different linkages must now be explored. 


\section{Experimental Section}

General Methods. Evaporations were done on a Berkeiey rotary evaporator using an oil punp for reduced pressure. The NMR spectra were obtained on the University of California, Davis, $500 \mathrm{MHz}$ spectrometer and the chemical shifts are expressed in ppm relative to an external TSP standard. High pressure liquid chromatography (HPLC) was done on en Altex system consisting of two Model 110A pumps, a Model 115-10 UV/vis detector, and a Model 420 microprocessor.

Preparation of the Phycoerythrobilin Peptide B-3T. The conditions employed for the tryptic digestion of Porphyridium cruentum B-phycoerythrin and for the isolation and carboxymethylation of peptide $\beta-3$ were those described by Lundell et al. ${ }^{4}$ Carboxymethylated peptide $8-3$ $(4.9 \mu \mathrm{mol})$ in $1 \mathrm{M}$ acetic acid was dialyzed overnight at $4^{\circ} \mathrm{C}$ against $10 \mathrm{mM} \mathrm{HCl}$ (Spectra/Por dialysis tubing, MW cut-off 3,500). After dialysis. the peptide solution $(12 \mathrm{~mL})$ was adjusted to $\mathrm{pH} 7.6 \mathrm{by}$ the addition of 1/10 volume of ? M Tris- $\mathrm{Cl}, \mathrm{pH} \mathrm{8,} \mathrm{and} \mathrm{calcium} \mathrm{chloride} \mathrm{was} \mathrm{added} \mathrm{to} \mathrm{a}$ final concentration of $5 \mathrm{mM}$. Thermolysin $(375 \mu \mathrm{g})$ was added and the mixture was incubated under $\mathrm{N}_{2}$ in the dark at $37^{\circ} \mathrm{C}$ for 150 min. A second aliquot of thermolysin $(375 \mu \mathrm{g})$ was then added and the digestion was allowed to proceed for a further 190 min under the same conditions. The reaction was terminated by acidification to $\mathrm{pH} 2$ with $7 \mathrm{M} \mathrm{HCl}$.

The digest was applied to a column $(1.5 \times 20 \mathrm{~cm})$ of SP-Sephacex $\mathrm{C}-25$ in $0.05 \mathrm{M}$ sodium phosphate, $\mathrm{pH} 2.5$, and the column was developed with a linear gradient $(500 \mathrm{~mL})$ of 0.0 to $0.4 \mathrm{M} \mathrm{NaCl}$ in $0.05 \mathrm{M}$ sodium phosphate, pH 2.5 at a flow rate of $30 \mathrm{~mL} / \mathrm{hr}$. A single peak of bilincontaining material was eluted at apprc.:imately $0.21 \mathrm{M} \mathrm{NeCl}$. Amino acid 
analysis data and UV/vis spectral analysis are available. ${ }^{4}$

For further studies, the eluate from the SP-Sephadex column was adsorbed onto a column of LiChrosorb C-18, washed extensively with $10 \mathrm{~m} / \mathrm{s}$ aqueous formic acid, and then eluted with $60 \%$ acetonitrile $/ 40 \% 10 \mathrm{~mm}$ aqueous formic acid. The acetonitrile was removed under a stream of nitrogen. HPLC analysis of the desalted pcptide B-3T is shown in Figure 2.

Sample Preparation for NMR. The chromc jeptide in 10 rill aqueous formic acid was concentrated under reduced pressure by rotary evaporation to $0.5 \mathrm{~mL}$ and dituted with $10 \mathrm{~mL}$ of $10 \mathrm{mM}$ trifluoroacetic acid (IFA) in $99.8 \% \mathrm{D}_{2} \mathrm{O}$ (made by adding $50 \mu \mathrm{mol}$ of trifluoroacetic anhydride to $10 \mathrm{~mL}$ of $\left.99.8 \% \mathrm{D}_{2} \mathrm{O}\right)$. The solution was stirred for $1 \mathrm{hr}$ at room temperature and concentrated as before to $0.5 \mathrm{~mL}$. This same procedure was repeated twice and then twice using $1.0 \mathrm{~mL}$ of $99.96 \% \mathrm{D}_{2} \mathrm{O}$. Finally, the NAP? sample kas prepared in $1.0 \mathrm{~mL}$ of $10 \mathrm{mil}$ TFA in $99.996 \% \mathrm{D}_{2} \mathrm{C}$ at a concentration of $2.2 \mathrm{~m}:$.

Secondary Ion Mass Spectrometry. An aliquot of the chromopepitide in 10 rivi aqueous TFA representing approximately $3 \mu \mathrm{g}$ was applied directly to the glycerol matrix. Scans were done at 30 seconds/decade." 

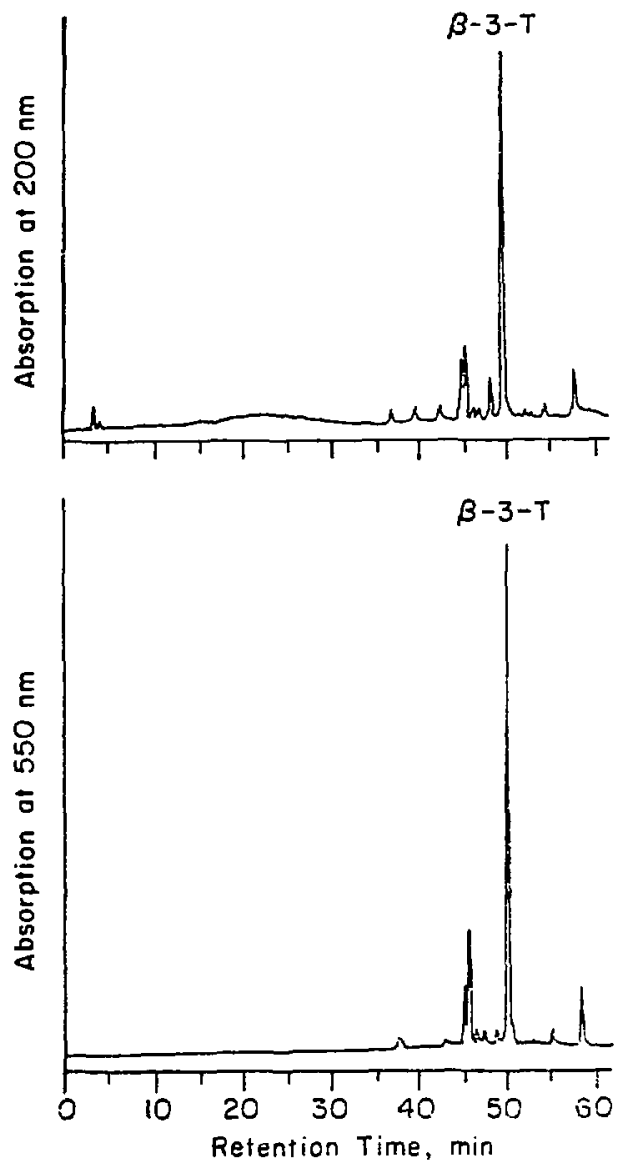

Fioure 2. High pressure liquid chromatography of the physoerythrobilipeptiue $\hat{5}-3 T$ on an Ultrashere ODS column (4.6×250 mm, $5 \mu \mathrm{m})$ using a 50 minute gradient $(70 \%-30 \%)$ af $\mathrm{CH}_{3} \mathrm{CN}$ in $0.70 \mathrm{MaH}_{2} \mathrm{PO}_{4}$ buffer, f4 2.1 with a $1.0 \mathrm{n!} ! /$ min flow rute. 


\section{Acknowledgement}

This research was supported in part by the Division of Biological Energy Conversion and Conservation, U.S. Department of Energy under Contract No. DE-ACO3-76SF00098, the National Institute of General Medical Sciences, DHHW, grant GM 28994, and National Science Foundation grant NSF PCM 82-08158. The $500 \mathrm{MHz}$ NMR studies were carried out at the University of California, Davis, NMR Facility, under the auspices of National Science Foundation grant CHE 79-04932. The secondary ion mass spectral data were obtained from the Bioorganic Biomedical Mass Spectrometry Resource (A.L. Burlingame, Director), University of California, supported by NIH Division of Research Resources grant RR01614. 


\section{References}

(1) Gantt, E.; Lipschu1tz, C.A. Biochenistry 1974, 13, 2960.

(2) Glazer, A.N.; Hixson, C.S. J. Biol. Chem. $\underset{\sim}{1977}, 252,32$.

(3) Abad-Zabatero, C.; Fox, J.L.; Hackert, H.L. Biochem. Biophys. Res. Commun. 1977, 78, 266.

(4) Lunde11, D.J.; Glazer, A.N.; DeLange, R.J.; Brown, D.M. J. Biol. Chem. 1983, submitted.

(5) Muckle, G.; Otto, J.; Rudiger, W. Hoppe-Seyler's Z. Physiol. Chsm. $1978,359,345$.

(6) Sidler, W.; Fuglistaller, P.; Gysi, J.; Isker, E.; Zuter, H. "Photosynthasis I1I, Structure and Molecular Organization of the Photosynthes is Apparatus"; Akoyunoglou, G., Ed.; Ba?aban Internat.

Sci. Service: Philadelphia, 2981; p 583.

(3) Williams, D.ti.; Bradiey, C.V.; Santikarn, S.; Bojesen, G. Biuchem. J. 1982, 201, 105.

(9) Mari'is, H.R.; Panico, M. Biochem. Biophys. Res. Cormun. 1987, 10?, $E 23$.

(10) C Carra, P.; Murphy, R.F.; Killilea, S.D. Biointin. J. 1980, 187, 203.

(11) Gossauer, A. Tetrahedron $\underset{\sim}{1933}, 39,1933$.

(12) Lagariàs, J.C.; Glazer, A.N.; Rapoport, H. J. Ann. Chiem. Joc. $1972,101,5030$

(13) Gosszuer, A.; Weller, J.-P. J. Am. Chem. Soc. 1978, 100, 5928.

(14) O Caria. P.; O hEocha, C. "Chemistry and Biochemistry of Plant Fig̣nents"; Goodwin, T.W., Ed.; Acadernic Press: New York, 1976; yol. 1, p 328. 
This report was done with support from the Department of Energy. Any conclusions or opinions expressed in this report represent solely thase of the author(s) and not necessarily those of The Regents of the University of California, the Lawrence Berkeley Laboratory or the Department of Energy.

Reference to a company or product name does not inply approval or recommendation of the product by the University of California or the U.S. Department of Energy to the exclusion of others that may be suilable. 